\title{
THE PANCHROMATIC HUBBLE ANDROMEDA TREASURY
}

\author{
Julianne J. Dalcanton ${ }^{1}$, Benjamin F. Williams ${ }^{1}$, Dustin Lang $^{2}$, Tod R. Lauer ${ }^{3}$, Jason S. Kalirai ${ }^{4}$, Anil C. Seth ${ }^{5}$, \\ Andrew Dolphin $^{6}$, Philip Rosenfield ${ }^{1}$, Daniel R. Weisz ${ }^{1}$, Eric F. Bell ${ }^{7}$, Luciana C. Bianchi ${ }^{8}$, Martha L. Boyer ${ }^{4}$, \\ Nelson Caldwell ${ }^{9}$, Hui Dong ${ }^{3}$, Claire E. Dorman ${ }^{10}$, Karoline M. Gilbert ${ }^{1,20}$, Léo Girardi $^{11}$, Stephanie M. Gogarten ${ }^{1}$, \\ Karl D. Gordon ${ }^{4}$, Puragra Guhathakurta ${ }^{10}$, Paul W. Hodge ${ }^{1}$, Jon A. Holtzman ${ }^{12}$, L. Clifton Johnson ${ }^{1}$, \\ Søren S. Larsen ${ }^{13}$, Alexia Lewis ${ }^{1}$, Jason L. Melbourne ${ }^{14}$, Knut A. G. Olsen ${ }^{3}$, Hans-Walter Rix ${ }^{15}$, Keith Rosema ${ }^{16}$, \\ Abhijit Saha ${ }^{3}$, Ata Sarajedini ${ }^{17}$, Evan D. Skillman ${ }^{18}$, and Krzysztof Z. StaneK ${ }^{19}$ \\ ${ }^{1}$ Department of Astronomy, University of Washington, Box 351580, Seattle, WA 98195, USA \\ ${ }^{2}$ Department of Astrophysical Sciences, Princeton University, Princeton, NJ 08544, USA \\ ${ }^{3}$ National Optical Astronomy Observatory, 950 North Cherry Avenue, Tucson, AZ 85719, USA \\ ${ }^{4}$ Space Telescope Science Institute, 3700 San Martin Drive, Baltimore, MD 21218, USA \\ ${ }^{5}$ Department of Physics \& Astronomy, University of Utah, Salt Lake City, UT 84112, USA \\ ${ }^{6}$ Raytheon Company, 1151 East Hermans Road, Tucson, AZ 85756, USA \\ ${ }^{7}$ Department of Astronomy, University of Michigan, 500 Church St., Ann Arbor, MI 48109, USA \\ ${ }^{8}$ Department of Physics and Astronomy, Johns Hopkins University, Baltimore, MD 21218, USA \\ ${ }^{9}$ Harvard-Smithsonian Center for Astrophysics, 60 Garden Street, Cambridge, MA 02138, USA \\ ${ }^{10}$ University of California Observatories/Lick Observatory, University of California, 1156 High St., Santa Cruz, CA 95064, USA \\ ${ }^{11}$ Osservatorio Astronomico di Padova-INAF, Vicolo dell'Osservatorio 5, I-35122 Padova, Italy \\ 12 Department of Astronomy, New Mexico State University, Box 30001-Department 4500, 1320 Frenger Street, Las Cruces, NM 88003, USA \\ ${ }^{13}$ Department of Astrophysics, IMAPP, Radboud University Nijmegen, P.O. Box 9010, 6500 GL Nijmegen, The Netherlands \\ ${ }^{14}$ Caltech Optical Observatories, Division of Physics, Mathematics and Astronomy, Mail Stop 301-17, California Institute of Technology, Pasadena, CA 91125, USA \\ ${ }^{15}$ Max Planck Institute for Astronomy, Koenigstuhl 17, 69117 Heidelberg, Germany \\ ${ }^{16}$ Random Walk Group, 5209 21st Ave. N.E., Seattle, WA 98105, USA \\ ${ }^{17}$ Department of Astronomy, University of Florida, Gainesville, FL 32611, USA \\ ${ }^{18}$ Minnesota Institute for Astrophysics, University of Minnesota, 116 Church Street SE, Minneapolis, MN 55455, USA \\ ${ }^{19}$ Department of Astronomy, The Ohio State University, 140 West 18th Avenue, Columbus, OH 43210, USA \\ Received 2012 February 17; accepted 2012 March 29; published 2012 May 31
}

\begin{abstract}
The Panchromatic Hubble Andromeda Treasury is an ongoing Hubble Space Telescope Multi-Cycle Treasury program to image $\sim 1 / 3$ of M31's star-forming disk in six filters, spanning from the ultraviolet (UV) to the nearinfrared (NIR). We use the Wide Field Camera 3 (WFC3) and Advanced Camera for Surveys (ACS) to resolve the galaxy into millions of individual stars with projected radii from 0 to $20 \mathrm{kpc}$. The full survey will cover a contiguous $0.5 \mathrm{deg}^{2}$ area in 828 orbits. Imaging is being obtained in the F275W and F336W filters on the WFC3/UVIS camera, F475W and F814W on ACS/WFC, and F110W and F160W on WFC3/IR. The resulting wavelength coverage gives excellent constraints on stellar temperature, bolometric luminosity, and extinction for most spectral types. The data produce photometry with a signal-to-noise ratio of 4 at $m_{\mathrm{F} 275 \mathrm{~W}}=25.1, m_{\mathrm{F} 336 \mathrm{~W}}=24.9, m_{\mathrm{F} 475 \mathrm{~W}}=27.9$, $m_{\mathrm{F} 814 \mathrm{~W}}=27.1, m_{\mathrm{F} 110 \mathrm{~W}}=25.5$, and $m_{\mathrm{F} 160 \mathrm{~W}}=24.6$ for single pointings in the uncrowded outer disk; in the inner disk, however, the optical and NIR data are crowding limited, and the deepest reliable magnitudes are up to 5 mag brighter. Observations are carried out in two orbits per pointing, split between WFC3/UVIS and WFC3/IR cameras in primary mode, with ACS/WFC run in parallel. All pointings are dithered to produce Nyquist-sampled images in F475W, F814W, and F160W. We describe the observing strategy, photometry, astrometry, and data products available for the survey, along with extensive testing of photometric stability, crowding errors, spatially dependent photometric biases, and telescope pointing control. We also report on initial fits to the structure of M31's disk, derived from the density of red giant branch stars, in a way that is independent of assumed mass-to-light ratios and is robust to variations in dust extinction. These fits also show that the $10 \mathrm{kpc}$ ring is not just a region of enhanced recent star formation, but is instead a dynamical structure containing a significant overdensity of stars with ages $>1$ Gyr.
\end{abstract}

Key words: galaxies: individual (M31) - galaxies: stellar content - stars: general - stars: imaging

Online-only material: color figures

\section{INTRODUCTION}

Our quest to understand the universe relies on detailed knowledge of physical processes that can only be calibrated nearby. It is impossible to interpret observations across cosmic time without an underlying understanding of stellar evolution, star formation, the initial mass function (IMF), the extinction law, and the distance scale, all of which require detailed studies

\footnotetext{
${ }^{20}$ Hubble Fellow
}

of individual stars and the interstellar medium (ISM) on subkiloparsec scales.

When the needed studies of stars and gas are carried out in the Milky Way (MW), they frequently face complications from line-of-sight reddening, uncertain distances, and background/ foreground confusion. As such, it is sometimes easier to constrain physical processes in external galaxies, which are free of the projection effects that can plague MW studies. Not only are observations of external galaxies more straightforward to interpret, but they can also be placed in the larger context of the 
surrounding environment (i.e., the ISM, metallicity, and star formation rate, SFR). Galaxies in the Local Group therefore offer an excellent compromise between being close enough to resolve relatively faint stars, while being distant enough to unveil the complex processes that govern star and galaxy evolution in their full galactic context.

Unfortunately, even the nearest massive galaxies have sufficiently high stellar surface densities that severe crowding compromises the detection of fainter, more age-sensitive stellar populations, allowing only the brightest stars to be studied with typical ground-based angular resolution in high-surface brightness regions of galaxy disks (e.g., Massey et al. 2006). However, with the high angular resolution available from Hubble Space Telescope (HST), we have the potential to resolve millions to billions of stars within the Local Group, grouped into galaxies with a common distance and foreground extinction. These stars, along with their ancestors and descendants (e.g., molecular clouds, H II regions, variable stars, X-ray binaries, supernova (SN) remnants, etc.), provide transformative tools for strengthening the foundation on which knowledge of the distant universe is based.

Within the Local Group, the Andromeda galaxy (M31) offers the best proxy for the properties of more distant galaxies. It is massive (sampling above the characteristic stellar mass $(3-5 \times$ $10^{10} M_{\odot}$ ) over which rapid systematic changes in galaxies' stellar populations and structure occur; e.g., Kauffmann et al. 2003), hosts spiral structure, and contains the nearest example of a traditional spheroidal component (outside the MW). M31 is also representative of the environments in which typical stars are found today. More than half of all stars are currently found in the disk and bulges of disk-dominated galaxies like M31 (Driver et al. 2007), and more than $3 / 4$ of all stars in the universe have metallicities within a factor of two of solar (Gallazzi et al. 2008), comparable to the typical metallicities of stars in M31.

In addition to its dominant solar-metallicity population of young stars, M31 also contains significant populations of older super-solar-metallicity stars in its bulge, and sub-solarmetallicity stars in its outskirts (e.g., Sil'chenko et al. 1998; Lauer et al. 2012; Brown et al. 2003; Worthey et al. 2005; Kalirai et al. 2006a; Chapman et al. 2006; Fan et al. 2008, and references therein), making M31 a superb laboratory for constraining stellar evolution models across at least an order of magnitude in iron abundance. Moreover, because of its high mass, M31 contains $\gtrsim 90 \%$ of the stars in the Local Group (outside the MW), making it ideal for generating samples of sufficient size that Poisson statistics are negligible and even rare phenomena are well represented. Finally, the stars in M31 are bright enough to be accessible spectroscopically, allowing one to augment imaging observations with spectroscopy, providing measurements of the kinematics, metallicities, spectral types, and physical parameters of star clusters and massive mainsequence, asymptotic giant branch (AGB), and red giant branch (RGB) stars.

We have therefore undertaken a new imaging survey of M31's bulge and disk using HST. The Panchromatic Hubble Andromeda Treasury (PHAT) survey is being carried out as a "Multi-Cycle Treasury" program to image a large contiguous area in M31, building upon the existing ground-based studies that probe M31's most luminous stars (most recently, Magnier et al. 1992; Massey et al. 2006; Mould et al. 2004; Skrutskie et al. 2006). The survey uses HST's new instrumentation to provide spectral coverage from the ultraviolet (UV) through the nearinfrared (NIR), with which one can effectively measure the bolo- metric luminosity, spectral energy distribution, and morphology of most astrophysical objects. Broadband coverage allows one to constrain the mass, metallicity, and ages of stars, even in the presence of extinction. Other objects such as background active galactic nuclei (AGNs), planetary nebulae (PNe), X-ray binaries, and SN remnants, whose bolometric luminosity peaks outside the accessible wavelength range, will still have distinctive spectral energy distributions within the HST filter coverage. Thus, the broad wavelength coverage of the survey enables full characterization of stars, their evolved descendants, and useful background sources. When complete, the survey should contain data on more than 100 million stars, comparable to the number found in the Sloan Digital Sky Survey (SDSS).

The legacy and scientific value of our survey is rich and diverse. The survey data can be used to (1) provide the tightest constraints to date on the slope of the stellar IMF above $\gtrsim 5 M_{\odot}$ as a function of environment and metallicity; (2) provide a rich collection of clusters spanning wide ranges of age and metallicity, for calibrating models of cluster and stellar evolution (e.g., Johnson et al. 2012); (3) characterize the history of star formation as a function of radius and azimuth, revealing the spiral dynamics, the growth of the galaxy disk and spheroid in action, and the role of tidal interactions and stellar accretion; (4) create spatially resolved UV-through-NIR spectral energy distributions of thousands of previously cataloged X-ray binaries, SN remnants, Cepheids, PNe, and Wolf-Rayet stars, allowing full characterization of these sources; (5) constrain rare phases of stellar evolution (e.g., Rosenfield et al. 2012); and (6) provide rich probes of the gas phase and its interaction with star formation, by using sub-arcsecond extinction mapping as a probe of the molecular gas, and by comparing parsec-scale gas structures to the recent history of star formation and stellar mass loss. In the future, the survey will provide the fundamental baseline for characterizing sources in transient surveys (e.g., PanSTARRS, LSST) both by using direct identification of counterparts, and by associating transients with the properties of the surrounding stellar population, when the counterpart is undetected even at HST's depth and resolution. The survey will also catalog hundreds of UV-luminous background AGNs, which can be used as absorption line probes of the detailed physics of the ISM in future UV spectroscopic observations, and as a reference frame for proper motion studies.

In this paper, after giving a brief history of stellar population studies in M31 (Section 2), we present the survey design (Section 3) and data reduction (Section 4), along with a thorough characterization of the data quality. In Section 5, we present initial color-magnitude diagrams (CMDs) covering several kiloparsec-scale contiguous regions, located in the bulge, two major star-forming rings, and the outer disk. We also present a preliminary analysis of the structure of M31's disk, based on counts of RGB stars in the NIR.

\section{THE HISTORY OF M31 RESOLVED STELLAR POPULATION STUDIES}

The recognition of the presence of different stellar populations in M31 began when Hubble (1929) noted that the outer parts of the disk could be resolved into stars, while the central region showed only unresolved light, despite clear spectroscopic evidence that it was made up of stars. When Baade carried out his 20 year study of M31 (see Baade \& Payne-Gaposchkin 1963), he used red-sensitive plates to show that the central regions could indeed be resolved into stars, and had properties similar to those 
of Galactic globular clusters. Based on these M31 studies, Baade developed the influential concept of Populations I and II, which formed the foundation of subsequent population studies. The central areas of M31 were made up of red, low-luminosity stars (Population II) and the main disk contained luminous blue stars (Population I). At the time of Baade's work, theoretical stellar evolution calculations (Schwarzschild 1965) were beginning to provide models that nicely fit the kinds of patterns found observationally by Baade, allowing the empirical facts of different stellar properties to be turned into quantitative population histories. Baade concluded that the central bulge of M31 was made up of very old stars, while the bright blue arms were young. He mapped out the structure of these arms and showed that the most luminous areas were fragments of arms located at radial distances of between 8 and $12 \mathrm{kpc}$ (see also work by Arp 1964). Subsequent work by many astronomers (see van den Bergh 1991; Hodge 1992 for references) confirmed this pattern, but showed that the dichotomy of just two populations was too simple. Population studies of the disk of M31 by Williams (2003), for example, showed that the populations vary across the disk, implying different histories of star formation within the broader classification of Population I. The structures seen between 8 and $12 \mathrm{kpc}$ are now thought to be a ring of star formation at $\sim 10 \mathrm{kpc}$ (Habing et al. 1984; Gordon et al. 2006), based in large part on observations of M31's ISM.

Global population studies were sparse in the later parts of the 20th century, in spite of major wide-area ground-based CCD surveys of M31's bright stellar content (e.g., Magnier et al. 1992). Most papers instead looked at smaller units, e.g., globular clusters (e.g., Rich et al. 1996), OB associations (e.g., Massey et al. 1986; Haiman et al. 1994; Hunter et al. 1996), and individual HST fields (e.g., Rich \& Mighell 1995). After the turn of the century, large new catalogs of M31's stars were produced in the optical (Massey et al. 2006) and infrared (Skrutskie et al. 2006; Mould et al. 2008), but much of the more recent work on stellar populations concentrated on the halo and on extended disk stars (e.g., Cuillandre et al. 2001; Durrell et al. 2001; Ferguson \& Johnson 2001; Sarajedini \& Van Duyne 2001; Rich et al. 2004; Brown et al. 2006, 2007, 2008, 2009b; Kalirai et al. 2006a, 2006b; Richardson et al. 2008; Bernard et al. 2012, and many others), and the population of the bulge (e.g., Saglia et al. 2010; Davidge 2001; Davidge et al. 2005; Sarajedini \& Jablonka 2005; Stephens et al. 2003; Olsen et al. 2006; Rosenfield et al. 2012), which holds special interest due to the presence of a significant super-solar-metallicity stellar population. Analyses of stellar clusters (e.g., Krienke \& Hodge 2007, 2008; Barmby et al. 2009; Hodge et al. 2009; Perina et al. 2010) and various $H S T$ pointings have also continued in earnest (Bellazzini et al. 2003).

These and other studies have confirmed a basic picture where M31 hosts a clear disk and bulge. However, there is evidence for more complex structures in the inner region (including a bar and a boxy peanut-shaped bulge, in addition to M31's classical bulge; Lindblad 1956; Stark 1977; Stark \& Binney 1994; Athanassoula \& Beaton 2006; Beaton et al. 2007), the disk (which shows a change in position angle at $\sim 18 \mathrm{kpc}$, most likely due to a warp; e.g., Walterbos \& Kennicutt 1988), and the outer disk and halo (most recently, Tempel et al. 2010; McConnachie et al. 2009). The disk contains ample evidence for recent star formation, confined largely to major spiral arms or the $10 \mathrm{kpc}$ ring. Near the ring, M31's current metallicity appears to be comparable to or higher than that of the Milky Way, with ambiguous evidence for a gradient in $\mathrm{O} / \mathrm{H}$; however, existing metallicity data is surprisingly sparse, spanning a limited range in radii with large 0.5 dex scatter in the metallicity at any given radius (Rubin et al. 1972; Dennefeld \& Kunth 1981; Blair et al. 1982; Zaritsky et al. 1994; Galarza et al. 1999; Venn et al. 2000; Smartt et al. 2001; Han et al. 2001; Trundle et al. 2002). Analyses of older stellar populations (RGB and PNe) suggest that the disk of M31 hosts a wide range of stellar metallicities (e.g., Worthey et al. 2005; Jacoby \& Ciardullo 1999; Richer et al. 1999, and references therein). The metallicity of older stars in M31's bulge likewise spans a wide range, but seems to reach super-solar metallicities in the very center (e.g., Sil'chenko et al. 1998; Lauer et al. 2012, and references therein).

Somewhat surprisingly, the distance to M31 remained largely uncontroversial in the last two decades, unlike for the other galaxies in the Local Group, be it the Large Magellanic Cloud (e.g., Macri et al. 2006; van Leeuwen et al. 2007) or M33 (e.g., Bonanos et al. 2006; Scowcroft et al. 2009). For example, the Cepheid distance modulus to M31 of $\mu_{0}=24.41 \pm 0.08$ (Freedman \& Madore 1990; see also Riess et al. 2012 using data from the PHAT survey) agrees well with the red clump distance modulus of $\mu_{0}=24.47 \pm 0.06$ (Stanek \& Garnavich 1998), a value numerically identical to the tip of the red giant branch (TRGB) based distance of $\mu_{0}=24.47 \pm 0.07$ (McConnachie et al. 2005) and very close to the $\mu_{0}=24.44 \pm 0.12$ measured via a direct method using a detached eclipsing binary (Ribas et al. 2005). Given such too-good-to-be-true agreement one would normally suspect a "bandwagon effect," but all these papers use different methods and different zero-point calibrations to derive their distances. We will therefore adopt an M31 distance modulus of $\mu_{\mathrm{M} 31,0}=24.45 \pm 0.05$ (physical distance of $d_{\mathrm{M} 31}=776 \pm 18 \mathrm{kpc}$ ) in this paper.

\section{SURVEY DESIGN}

The optimal survey design for efficiently imaging a large portion of M31 requires finding solutions to several problems. These include determining the best portion of the galaxy to image, the best filters to use, and the best design of the exposure sequences. These in turn require identifying the best way to efficiently tile large areas with two cameras operating in parallel, while maximizing the photometric depth and image resolution, given a limited total exposure time for any location. In this section we discuss the rationale for the choices we made to address all of these issues in a way that we hope has optimized the broad scientific utility of the program.

We start in Section 3.1 with the rationale for the location and geometry of the PHAT survey area. Section 3.2 gives the scientific rationale for six-filter coverage from the UV through the NIR, and describes the specific filter choices. Section 3.3 explains the exposure strategy for fitting observations in six filters into two orbits. Section 3.4 shows how the observations are dithered to optimize spatial resolution and repair detector defects. Section 3.5 describes how the two-orbit visits are packed into an efficient $3 \times 6$ mosaic of 18 pointings, building the 23 "bricks" that tile the survey area. We close in Section 3.6 with a brief discussion of a coordinated spectroscopic campaign.

\subsection{Areal Coverage}

We evaluated several possible schemes for mapping M31. We initially considered producing a complete map of M31's starforming disk, using only one orbit per pointing and a reduced number of filters. However, meeting the broadest set of scientific goals required more complete filter coverage, and thus longer exposures at each position. We therefore reduced our areal 
coverage to a generous quadrant of M31. Since spirals like M31 have fairly regular structure, we judged that a quadrant would be sufficient to characterize the galaxy while still covering enough area to allow statistically significant samples of previously cataloged objects, of star-forming regions across the widest possible range of star formation intensity and metallicity, and of azimuthal variations in the SFR. We specifically chose the northeast quadrant, which has the lowest internal extinction; the largest number of regions with unobscured, high-intensity star formation; and the least contamination from M32. However, the tiling is generous enough that a substantial fraction of the northwest quadrant is covered by the HST imaging as well.

The resulting survey area is a long, roughly rectangular region with a slight bend in the middle. The long axis of the survey begins at the center of the galaxy, covering much of the bulge, and extends to the northeast following the major axis of the disk out to the last obvious regions of star formation visible in GALEX imaging (see maps in Thilker et al. 2005). The short axis spans the minor axis of the galaxy, out to a comparable projected radius as the long axis, once M31's inclination is taken into account.

In richer detail, the survey area is contiguous and is divided into 23 sub-areas, called "bricks," which solve a number of problems related to the efficient design and operation of a large multi-year survey; we discuss the design of the bricks in detail in Section 3.5. In the present context they can be considered to be rectangular tiles that "pave" the complete survey area. The bricks are arranged into two strips that respectively comprise the northwest and southeast halves of the survey area. Their detailed orientation and positioning gives the edges of the survey area a somewhat serrated appearance. Maps of the brick positions are shown in Figure 1, superimposed on various multi-wavelength images.

The northern strip has 12 bricks extending along the major axis from the bulge to the outer disk. The bricks in the southern strip are aligned to complete coverage of the quadrant. The naming scheme is such that Brick 1 covers the bulge, with odd numbered bricks extending along the major axis. To increase the windows in which observations can be scheduled, Bricks 1-11 are observed with a slightly different orientation than Bricks 12-23. In practice, we use the brick designations as a shorthand for referring to different regions of interest within the survey.

The survey area samples the diversity of environments within M31. The southwest end of the survey encloses much of the bulge, including the nucleus and the dense stellar population that surrounds it. The extent of the survey along both the major and minor axes of M31 is sufficient to observe the transition of the bulge into the main disk of M31. Star formation is probed at many locations within the disk, the most notable being the $10 \mathrm{kpc}$ ring of strong star formation, which is tracked throughout the southern edge of the survey. The width of the survey area completely tracks the roll-off of this arm into the background disk, and on the southern side of the galaxy, the terminus of the star-forming disk itself, into the surrounding halo. Weaker arms of star formation just outside the bulge are sampled, as are weaker spiral arms well outside of the main ring of star formation. Between the zones of strong star formation, the survey samples the smooth background population of the disk over the entire major axis of M31. The northeast end of the survey also captures the termination of the disk and the transition into the halo. To a fair approximation, the bricks that tile the survey area typically sample one or two of these various features. Table 1 presents a brief description of the populations present in each brick.

Because of the large number of orbits required by this program, not all bricks can be observed in a single Cycle. We have therefore prioritized the bricks to maximize the possible science output in early years. Our year 1 priority was to complete bricks that sample the full radial extent of the galaxy along the major axis, focusing on the bulge and major starforming rings and/or spiral arms (Bricks 1, 9, 15, 21, followed by Bricks 17 and 23). Year 2 priorities were to sample the highintensity star-forming ring (Bricks 2, 8, 12, 14, and 22), and to increase the sampling of star-forming regions on the major axis (Brick 5). Year 3 priorities are to complete most observations of the major star-forming ring (Bricks 4, 6, 16, and 18), and to start building larger contiguous regions in the inner and outer galaxy (Bricks 3,19). Year 4 will be devoted to completing all remaining areas (Bricks 7, 10,11, 13, and 20). The status of observations (as of fall 2011) is given in Table 1.

\subsection{Filter Choices}

The choice of UV-through-IR filter coverage was driven by a number of goals. The need for two optical filters was obvious, given that optical HST imaging data have proved to be the most efficient route to deriving the deepest possible CMDs for the largest number of stars. Supplementing the optical data with two additional NIR filters allows one to extend stellar population studies to dusty regions, and to better constrain the bolometric fluxes of intrinsically cool stars in important evolutionary phases (AGB stars, carbon stars, red core helium-burning stars). Adding an additional two UV filters opens up science that can be done with hot stars, and in particular permits simultaneous constraints of effective temperature and extinction, when combined with measurements in optical filters (e.g., Bianchi 2007; Romaniello et al. 2002; Zaritsky 1999).

The choice of specific filters, shown in Figure 2, was made as follows.

In the UV, we adopted F336W as the filter giving the highest throughput. This filter is also immediately blueward of the Balmer break, giving the best constraint on its amplitude. For the bluer UV filter, we wanted to push to the shortest possible wavelengths to give the best constraints on stellar temperature for the hottest stars, and to provide the largest baseline for constraining the extinction law. However, the bluest wide WFC3/UVIS filters have relatively low efficiency, and moreover fall within the $2175 \AA$ dust extinction feature, which is known to vary dramatically with environment. We therefore adopted F275W as the bluest, high throughput UV filter that would not be significantly affected by variations in dust composition.

In the optical, we adopted the F814W filter, which has consistently offered the highest throughput for stellar populations studies. The choice of the bluer optical filter was more problematic, as several valid choices exist. The F606W filter offers very high throughput and has been widely used for previous stellar population studies. However, it is quite red, providing a limited color baseline in the optical, and weaker constraints on the amplitude of the Balmer break (when combined with F336W). The F555W filter provides a wider color baseline than F606W and has also been used for a large number of HST stellar population studies. However, it is significantly narrower, and thus has much lower throughput. We therefore rejected these two filters and adopted the F475W filter (approximately SDSS- $g$ ). This filter is as broad as F606W, but is bluer than both F606W and F555W, providing much better color separation from F814W. 

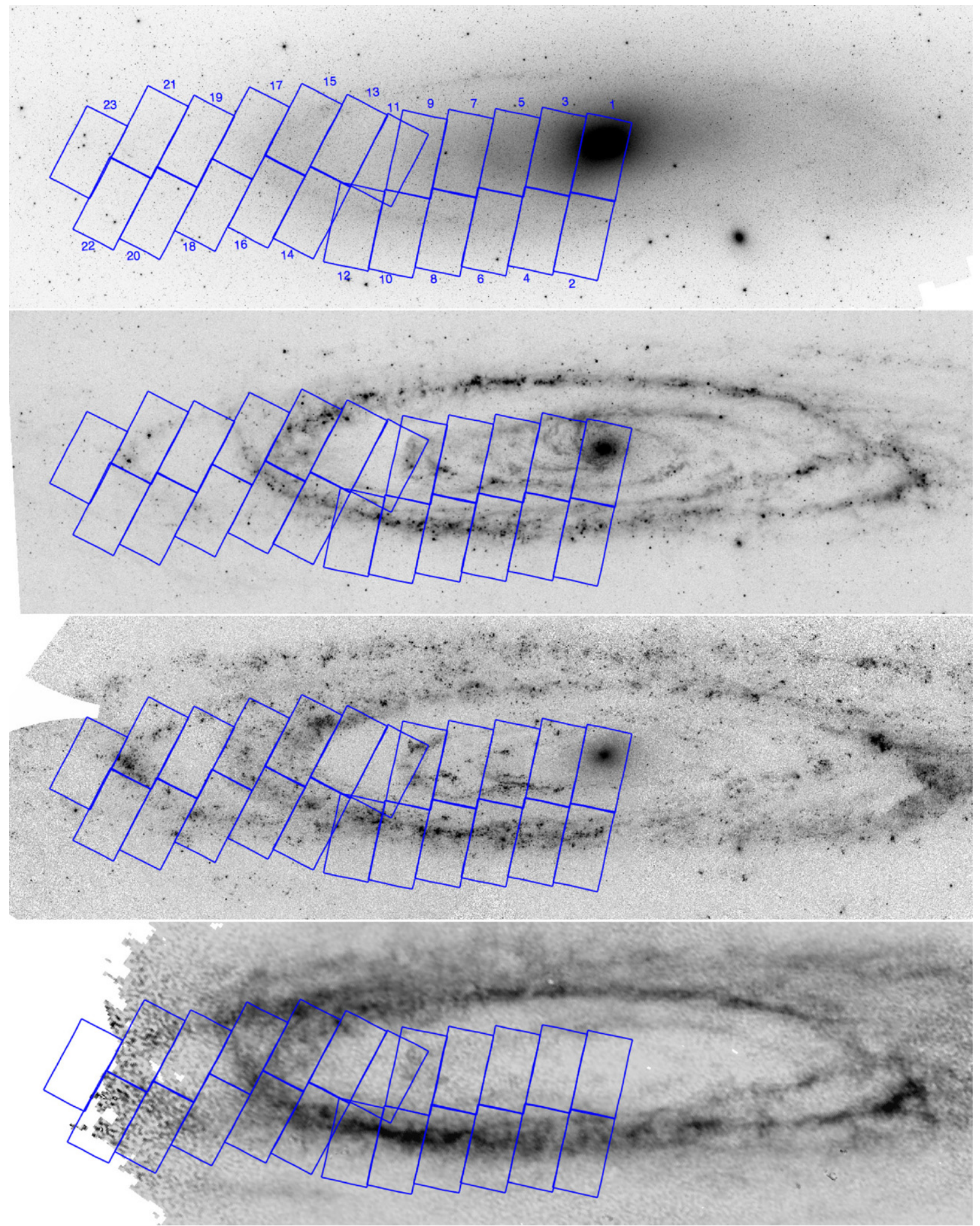

Figure 1. Location of PHAT "bricks," superimposed on a Spitzer $3.6 \mu \mathrm{m}$ image (Barmby et al. 2006), $24 \mu \mathrm{m}$ image (Gordon et al. 2006), GALEX FUV image (Thilker et al. 2005), and Westerbork H I image (Brinks \& Shane 1984), shown from top to bottom, respectively. The numbering scheme for the bricks is shown in the top panel; odd numbers are along the major axis, starting with Brick 1 on the bulge.

(A color version of this figure is available in the online journal.)

When combined with F336W, it provides good constraints on the Balmer break. The only cost is some loss in depth for intrinsically red stars (i.e., on the RGB). However, since much of the disk is crowding limited, this limitation is not severe at most pointings.
For the NIR, we use the F110W and F160W filters. These two filters provide the highest throughput with the Wide Field Camera 3 (WFC3)/IR camera and have been used successfully in our previous SNAP survey of stellar populations in nearby galaxies (Dalcanton et al. 2012). The only drawback with this 
Table 1

Timing of Brick Observations

\begin{tabular}{|c|c|c|c|c|c|c|}
\hline \multirow[t]{2}{*}{ Brick } & \multirow[t]{2}{*}{ PID } & \multicolumn{2}{|c|}{ WFC3 East and ACS West } & \multicolumn{2}{|c|}{ WFC3 West and ACS East } & \multirow[t]{2}{*}{ M31 Components Covered } \\
\hline & & Start & End & Start & End & \\
\hline 1 & 12058 & 2010 Dec 14 & $2010 \operatorname{Dec} 26^{\mathrm{a}}$ & 2010 Jul 21 & $2010 \mathrm{Jul} 25$ & Bulge, including nucleus \\
\hline 2 & 12073 & & & 2011 Jun 30 & 2011 Aug 3 & Outer bulge, NE star-forming arm \\
\hline 3 & 12109 & & & & & Outer bulge, inner disk, inner star-forming arms \\
\hline 4 & 12107 & & & & & NE star-forming arm \\
\hline 5 & 12074 & & & 2011 Jul 13 & 2011 Aug 3 & Inner-disk star formation \\
\hline 6 & 12105 & & & & & NE star-forming arm \\
\hline 7 & 12113 & & & & & Inner-disk star formation \\
\hline 8 & 12075 & & & $2011 \mathrm{Jul} 26$ & 2011 Aug 7 & NE star-forming arm; intense star formation; outer disk \\
\hline 9 & 12057 & 2010 Dec 30 & 2011 Jan 1 & $2010 \mathrm{Jul} 12$ & 2010 Jul 16 & Inner-disk star formation; significant overlap with brick 11 \\
\hline 10 & 12111 & & & & & NE star-forming arm; outer disk \\
\hline 11 & 12115 & & & & & Smooth inter-arm disk; significant overlap with brick 9 \\
\hline 12 & 12071 & & & 2011 Jul 1 & $2011 \mathrm{Jul} 13$ & NE star-forming arm; outer disk \\
\hline 13 & 12114 & & & & & Smooth, mainly inactive inter-arm disk \\
\hline 14 & 12072 & & & $2011 \mathrm{Jul} 22$ & 2011 Aug 12 & NE star-forming arms; outer arm star formation \\
\hline 15 & 12056 & 2011 Jan 10 & 2011 Jan 25 & 2010 Aug 7 & 2010 Aug 15 & Richest sample of main-arm star formation \\
\hline 16 & 12106 & & & 2011 Aug 29 & 2011 Sep $3^{b}$ & Main and outer-arm star formation \\
\hline 17 & 12059 & 2010 Dec 29 & 2011 Jan 9 & 2011 Jul 26 & 2011 Aug 6 & End of main star-forming ring \\
\hline 18 & 12108 & & & 2011 Aug 21 & 2011 Aug 28 & Outer-disk star formation \\
\hline 19 & 12110 & & & & & Inactive region in outer disk \\
\hline 20 & 12112 & & & & & Some outer-disk star formation; transition to halo \\
\hline 21 & 12055 & 2011 Jan 10 & 2011 Jan 15 & $2010 \mathrm{Jul} 26$ & $2010 \mathrm{Jul} 28$ & Outer-disk region of strong star formation \\
\hline 22 & 12076 & & & 2011 Aug 14 & 2011 Aug 17 & Outside of disk; transition to halo \\
\hline 23 & 12070 & 2011 Jan 26 & 2011 Feb $9^{b}$ & 2011 Aug 8 & 2011 Aug 14 & Outside of disk; transition to halo \\
\hline
\end{tabular}

Notes.

a One orbit covering B01-F03/F06 for ACSF814W/UVIS was completed in 2011 January 3, after a guide star failure during the previous year's observing season.

${ }^{\mathrm{b}}$ One pointing has not yet been observed due to lack of guide stars (B16-F14/F17 for ACS/WFC3) or currently has invalid data due to failed guiding (B21-F16 for ACS); these observations are expected to be finished in the winter 2011/2012 observing season.

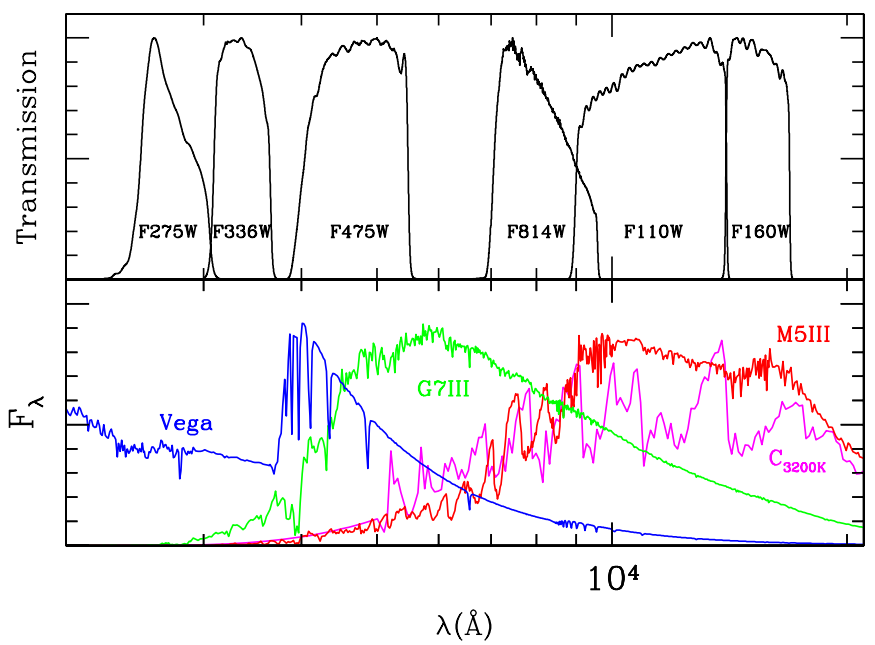

Figure 2. Relative transmission of the PHAT filter set (upper panel) as a function of wavelength. The lower panel shows a number of stellar spectra for comparison, including a Vega A-star spectrum (Bohlin 2007; blue), a G7III giant (Castelli \& Kurucz 2004; green), an M5III giant (Fluks et al. 1994; red), and a carbon star with a 3200K atmosphere (Loidl et al. 2001; red).

(A color version of this figure is available in the online journal.)

combination is the partial overlap of the F110W and F814W bandpasses. The only other feasible substitutes for the F110W filter would have been the F140W filter, which overlaps the F160W filter, and the F125W filter, which is much narrower than F110W, and which has less color separation and temperature sensitivity when paired with F160W.
Almost all of the PHAT filters have been used in calibration observations of nearby stellar clusters. The WFC3 Galactic Bulge Treasury Program (GO-11664; Brown et al. 2009a, 2010) has taken calibration observations in F814W, F110W, and F160W. F336W observations of the same clusters have been carried out in GO-11729 (PI: Holtzman). Our survey is executing observations of the same clusters in combinations of F275W and F475W filters to complete the calibrations of our filter set. Additional calibration in F336W, F475W, and F814W will be provided by GO-12257 (PI: Girardi) for intermediate-age Magellanic Cloud clusters.

The adopted filter set should allow us to make strong constraints on the effective temperature and extinction of the stars in our sample. With the UV + optical filters, we expect to be able to separate $T_{\text {eff }}$ and $E(B-V)$ with little degeneracy for both hot stars $\left(12,000 \mathrm{~K} \lesssim T_{\text {eff }} \lesssim 40,000 \mathrm{~K}\right.$; Massey et al. 1995; Romaniello et al. 2002; Bianchi 2007) and cooler stars (5500-6500 K; Zaritsky 1999). Inspection of reddening-free diagrams suggests that the optical + NIR combination will allow us to extend $E(B-V)$ constraints to cooler stars $\left(T_{\text {eff }}<5000 \mathrm{~K}\right)$ as well.

\subsection{Exposure Sequences}

The primary aims of our exposure plan are (1) imaging two filters per camera; (2) achieving Nyquist-sampled images through dithering where possible; and (3) avoiding saturation of bright sources. As we describe below, these goals are balanced against constraints on the number of images that can be downloaded when running WFC3 and Advanced Camera for Surveys (ACS) in parallel, and on limitations on the duration 

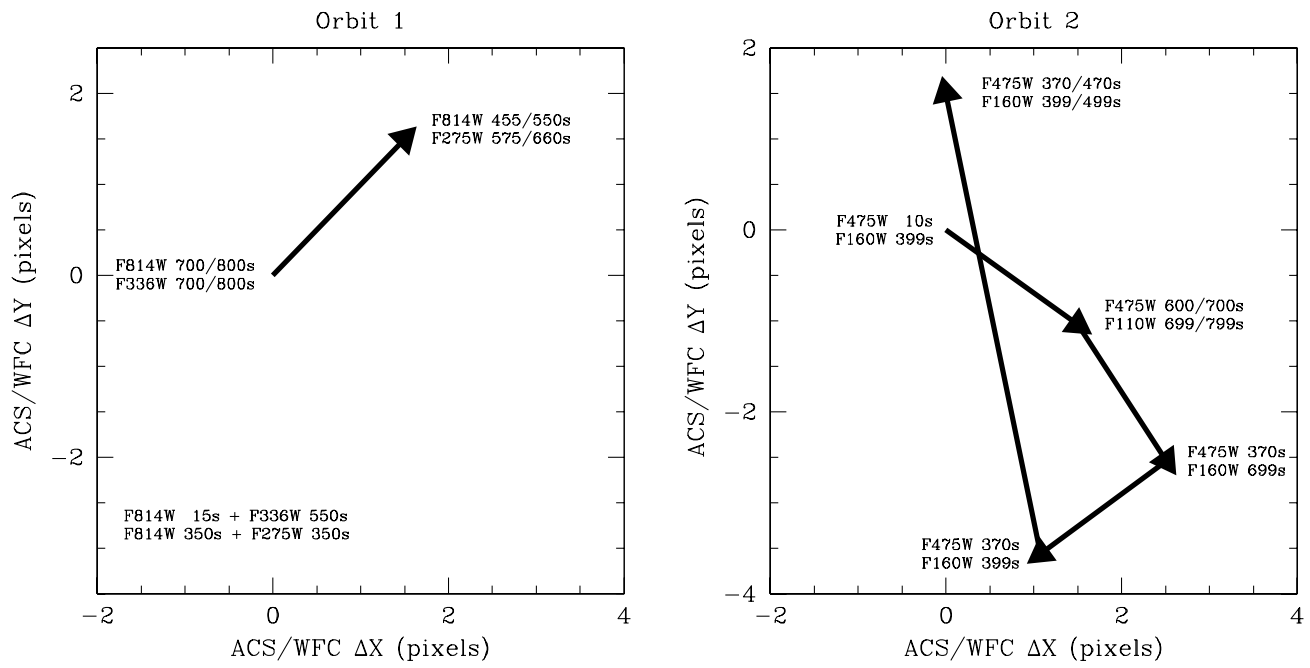

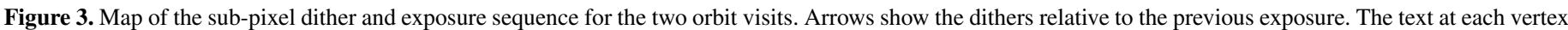

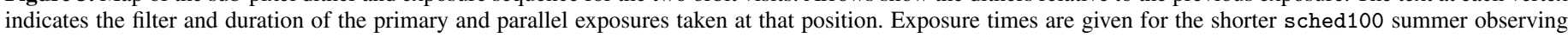

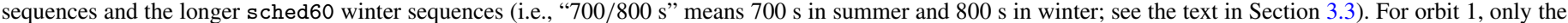

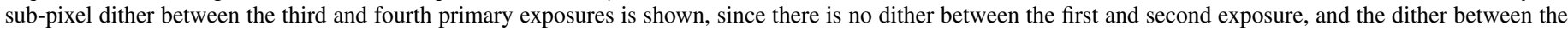
second and third is a large $\sim 37$ pixel dither to span the chip gap.

of an orbit. Because of the strains that this program puts on HST's observing schedule, the exposure sequences must fit within the shortest possible orbit duration ("sched100," i.e., an assumed duration that is schedulable for $100 \%$ of the orbits). This constraint maximizes the schedulability, particularly in the summer observing season (Section 3.5) which has a more restrictive scheduling window. In the winter observing season, we can relax the orbit length constraints to "sched60" (a duration that fits within $60 \%$ of the orbits), giving slightly longer exposures.

Scheduling observations in six filters requires two orbits, with the first orbit devoted to WFC3/UVIS+ACS/WFC and the second to WFC3/IR+ACS/WFC; this ordering minimizes persistence in the WFC3/IR channel (ISR WFC3 2008-33 by P. McCullough \& S. Deustua), by allowing more time for the persistent charge to decay. We run WFC3 in primary, and ACS in parallel. ${ }^{21}$

The strongest constraints on the observing sequence within each orbit come from the limited buffer space available on board the HST instruments. The ACS buffer can only hold one fullframe ACS/WFC image, and the WFC3 buffer can hold either two full-frame WFC3/UVIS images, or three WFC3/IR images (if the number of non-destructive reads in the latter is reduced to $\sim 10$ from the nominal 15 samples) before needing to be dumped. However, the time to dump the buffer is substantial ( $\sim 340 \mathrm{~s})$, which can lead to significant inefficiencies if the observing sequence does not allow the buffers to dump in parallel with the observations. This issue is particularly severe when running both imaging cameras simultaneously, because of the high data rates.

After extensive experimentation, we were able to find exposure sequences that allow four WFC3/UVIS exposures and four ACS/WFC exposures in the first orbit, and five WFC3/IR exposures and five ACS/WFC exposures in the second orbit, with minimal latencies due to buffer dumps. The resulting observing

\footnotetext{
21 Because the corrections for differential velocity aberration (ISR OSG-CAL-97-06 by C. Cox) by the pointing control software are optimized for the primary observations, the ACS parallels will not automatically be corrected for this milliarcsecond level effect. The affine corrections used for astrometry (Section 4.6) should provide adequate corrections, however.
}

sequence also fits in dithers between every pair of exposures, with the exception of the first two in the WFC3/UVIS + ACS/ WFC orbit (see Figure 3). This high observing efficiency comes at the expense of unequal exposure times for observations in a single filter, and fewer non-destructive reads in the WFC3/IR observations (to allow the buffer to hold more WFC3/IR images between dumps). The sequence is summarized in Table 2.

During the first orbit, the four WFC3/UVIS exposures follow the sequence: F336W (550s; 550s), F275W (350s; $350 \mathrm{~s}$ ), F336W (700s; $800 s)$, and F275W (575s; 660s), where the two numbers in parentheses indicate the exposure times in the summer sched100 and winter sched60 observing seasons, respectively. We obtained two exposures in each filter to enable cosmic-ray $(\mathrm{CR})$ rejection and cover the chip gap; three exposures per filter would have been preferable, but constraints on buffer dumps limited the observations to no more than four WFC3/UVIS exposure per orbit. Note that the majority of the chip gap is only imaged in one exposure per filter, making CR rejection more challenging in this region.

The four ACS/WFC exposures in the first orbit are all in F814W, with exposure times of $(15 \mathrm{~s} ; 15 \mathrm{~s})$, (350s; $350 \mathrm{~s})$, $(700 \mathrm{~s} ; 800 \mathrm{~s})$, and $(455 \mathrm{~s} ; 550 \mathrm{~s})$. The short $15 \mathrm{~s}$ F814W exposure is included to allow photometry for stars brighter than F814W 17.5..$^{22}$

During the second orbit, the five WFC3/IR exposures follow the sequence F160W (NSAMP $=9$, STEP200, 399 s; NSAMP = 9, STEP200, 399 s), F110W $($ NSAMP $=13$, STEP100, $699 \mathrm{~s}$; NSAMP $=11$, STEP200, 799 s), F160W (NSAMP = 9, STEP200, $399 \mathrm{~s} ;$ NSAMP $=9$, STEP200, 399s), F160W $($ NSAMP $=9$, STEP200, 399 s; NSAMP $=$ 9, STEP200, 399s), and F160W $($ NSAMP $=9$, STEP200, $399 \mathrm{~s} ;$ NSAMP $=11$, STEP100, 499 $\mathrm{s})$, where the numbers in parentheses give the number of samples, the MULTIACCUM exposure sequence, and resulting exposure

\footnotetext{
22 There is no equivalent short "guard" exposure in the UV, since the number of saturated stars was expected to be negligible (based on existing ground-based $U$-band data from the Massey et al. 2006 Local Group Survey), and the penalty in exposure time would be large for the unsaturated stars (given the small number of possible WFC3/UVIS exposures). In practice, it may be possible to pull out reasonable photometry from even the saturated stars (see ISR WFC3 2010-10 by R. Gilliand et al. for WFC3/UVIS and Anderson et al. 2008 for ACS/WFC).
} 
Table 2

Two-orbit Exposure Sequence

\begin{tabular}{|c|c|c|c|c|c|c|}
\hline $\begin{array}{l}\text { ACS } \\
\text { Filter }\end{array}$ & $\begin{array}{l}\text { Exp } \\
\text { (s) }\end{array}$ & $\begin{array}{l}\Delta x, \Delta y \\
\text { (pixels) }\end{array}$ & $\begin{array}{l}\text { WFC3 } \\
\text { Filter }\end{array}$ & $\begin{array}{l}\text { Exp } \\
\text { (s) }\end{array}$ & $\begin{array}{l}\Delta x, \Delta y \\
\text { (pixels) }\end{array}$ & Notes \\
\hline F814W & 15 & & F336W & 550 & & F814W guard \\
\hline F814W & 350 & & F275W & 350 & & \\
\hline \multicolumn{7}{|c|}{ Large 37 pixel Dither in $\Delta Y$ to Cover UVIS Chip Gap } \\
\hline F814W & $700 / 800$ & 0.00 & F336W & $700 / 800$ & & \\
\hline F814W & $455 / 550$ & 1.50 & F275W & $575 / 660$ & & ACS Nyquist dither \\
\hline F475W & 10 & & F160W & 399 & 0.00 & F475W guard \\
\hline F475W & $600 / 700$ & $0.00, \quad 0.00$ & F110W & $699 / 799$ & & ACS Nyquist dither \\
\hline F475W & 370 & $1.01,-1.50$ & F160W & 699 & $1.52, \quad 1.42$ & ACS+WFC3 Nyquist dither \\
\hline F475W & 370 & $0.48,-2.52$ & F160W & 399 & $1.94, \quad 1.56$ & ACS+WFC3 Nyquist dither \\
\hline F475W & $370 / 470$ & $-1.50, \quad 1.00$ & F160W & $399 / 499$ & $1.50,-0.01$ & ACS+WFC3 Nyquist dither \\
\hline
\end{tabular}

Notes. The F814W and WFC/UVIS exposures are obtained in the first orbit. The F475W and WFC/IR exposures are obtained in the second orbit. The dithers take place prior to the nominal exposure for which they are listed. Dithers are in pixels for the given camera. These are only specified for the sets of images for which dithers are designed to achieve the proper sub-pixel sampling needed to generate Nyquist-sampled images. The origin of the dithers is specified by the first image in the sequence. Several exposures have two values for the exposure time. The longer times are for images obtained with the "winter" sequence, which allows for longer orbital windows.

time for the summer sched100 and winter sched60 observing seasons, respectively. The STEP sequence was adopted to provide optimal sampling for the wide range of fluxes expected for observations of stellar populations. Since the MULTIACCUM sequence allows for $\mathrm{CR}$ rejection in an accumulated single image, multiple images are not needed. We therefore chose to use multiple pointings in F160W only, to allow the construction of a Nyquist-sampled image in this filter, at the expense of not being able to reject chip defects in F110W (the WFC3/IR "blobs" and the "death star"; ISR WFC3 2010-06 by N. Pirzkal). This trade-off was preferred, given the lower resolution of the WFC3/IR channel compared to the other cameras, and the partial overlap of the F110W and F814W bandpasses.

The five ACS/WFC exposures in the second orbit are all in F475W, with exposure times of (10s; $10 \mathrm{~s}),(600 \mathrm{~s} ; 700 \mathrm{~s})$, (370 s; $360 \mathrm{~s}),(370 \mathrm{~s} ; 360 \mathrm{~s})$, and (370 s; $470 \mathrm{~s})$. As for F814W, we include a short "guard" exposure to allow photometry of stars brighter than $\mathrm{F} 475 \mathrm{~W} \sim 18.5$.

The resulting total exposure times for each two-orbit visit are $(925 \mathrm{~s} ; 1010 \mathrm{~s})$ in F275W, (1250s; $1350 \mathrm{~s})$ in F336W, (1720s; $1900 \mathrm{~s})$ in F475W, $(1520 \mathrm{~s} ; 1715 \mathrm{~s})$ in F814W, $(699 \mathrm{~s} ; 799 \mathrm{~s})$ in F110W, and $(1596 \mathrm{~s} ; 1696 \mathrm{~s})$ in F160W, where the first and second numbers indicate the exposure times in the summer and winter observing seasons. The effective exposure times for the ACS observations will be at least a factor of two larger due to overlaps (see Figure 5), giving nearly two full orbits of exposure time in both F475W and F814W outside the chip gap.

\subsection{Dithering Strategy}

The exposure sequences were interleaved with small angle maneuvers to produce dithered images. Large dithers provide for the repair of detector defects (hot or bad pixels, missing columns, and so on), as well as coverage of the segments of M31 that fell into the ACS/WFC or WFC3/UVIS chip gaps in any single exposure. Smaller sub-pixel dithering enables well-sampled (Nyquist) images to be generated from a set of undersampled single exposures. Unfortunately, all of these tasks were difficult to satisfy simultaneously, given the limited number of exposures possible, the need to operate WFC 3 and ACS in parallel, and the geometric distortion present in all cameras. The distortion causes the angular pixel scale to vary with field position in the cameras, such that larger dither steps would produce a highly variable and generally non-optimal pattern of sub-pixel steps (once the integral portion of the step is subtracted) as a function of field location. Constructing Nyquistsampled images thus requires keeping the total size of the dithers small, which conflicts with the large dithers needed to bridge the CCD gaps.

With these qualifications, our primary goal was to use dithers to produce Nyquist-sampled images in as many filters as possible. We were able to do this in F475W, F814W, and F160W. The F475W and F160W filters each require four dithers such that the fractional portions of the shifts map out a $2 \times 2$ pattern of 0.5 pixel steps. In contrast, only two dither positions are required in F814W to achieve Nyquist sampling, given its larger point-spread function (PSF). Although the use of a single diagonal dither to produce Nyquist sampling is less intuitive than the traditional $2 \times 2$ dither pattern, a single diagonal shift can produce fractional offsets of 0.5 pixels in both $X$ and $Y$ at the same time, allowing the two images to be interlaced to produce a new image with a rectilinear sampling grid that is a factor $\sqrt{2}$ finer than the native ACS/WFC sampling. ${ }^{23}$ This approach is sufficient to ensure adequate sampling for $\mathrm{F} 814 \mathrm{~W}$, but not F475W, which requires $2 \times$ finer sub-sampling.

The dithers used to produce Nyquist sampling in the three aforementioned filters are summarized in Table 2. The specific dithers were designed so that where possible the correct fractional sub-sampling could be achieved in both ACS and WFC3 cameras simultaneously. The magnitude of the dithers is actually large enough so that small defects in the detectors can be stepped over, satisfying a second goal of the dithering, but not so large that geometric distortion ruins the accuracy of the shifts.

The design of the dither sequence was helped by the artful interplay of the location of the guard exposures and filter changes in the primary and parallel cameras, so that simultaneous sub-sampling dithers were not always required in both instruments at the same time. Of the four sub-sampling dithers needed for F475W and F160W, one dither is "exact" for each

\footnotetext{
23 A perfect analogue is the interlacing of black and white squares on a chess board. The black and white squares each form a regular grid with the sampling interval $\sqrt{2} \times$ larger than the spacing of the interlaced squares of the chess board.
} 
filter, while three are done simultaneously in the two filters and provide slightly non-optimal sub-pixel sampling. The single F814W sub-sampling dither occurs between a filter change in WFC3/UVIS and is optimal for that filter. In general, Nyquist images can still be readily constructed from dithers that stray significantly from the optimal sub-pixel steps (Lauer 1999), a consideration that must be allowed for, even with an optimally designed sequence, since the HST Fine Guidance Sensor (FGS) has limited accuracy in executing the dithers.

The dithers in the ACS F475W and F814W filters are not large enough to fill in the gap between the two CCDs in that camera. However, since every region that falls in the chip gap in one brick will be imaged again in another brick, completely filled coverage with the ACS can still be achieved. We thus have chosen to emphasize Nyquist sampling in all ACS filters at the expense of depth in the small portion of the survey that falls into the chip gaps.

Likewise, while the dithers in F160W are large enough to counter defects of one or two pixels in extent, the shifts are unfortunately not large enough to completely sample over the "IR blobs" or the "death star," which have characteristic sizes of $10-15$ pixels and 51 pixels, covering a total of $\sim 700$ pixels and $\sim 2000$ pixels, respectively (ISR WFC3 2010-06 by N. Pirzkal). We have found that the regions of the IR blobs can still produce adequate photometry, although with slightly higher uncertainties due to the $5 \%-30 \%$ reduction in sensitivity in the area of the blobs. The only area then that completely lacks WFC3/IR data is the $0.2 \%$ of the field covered by the WFC3/IR "death star." Again, we consider the benefits of obtaining Nyquist sampling over most of the survey in F160W to outweigh the sacrifice of a small portion of the area lost to unrepaired defects.

In contrast, we could not obtain the four-point dither pattern required to construct Nyquist-sampled images in either WFC3/UVIS filter. However, we do not expect this to be a significant scientific limitation, given that the WFC3/UVIS data are not crowding limited, and that any star detectable in WFC3/UVIS will also be detected in the Nyquist-sampled F475W images. Again, in contrast to the ACS/WFC, we did include a single large dither of $\sim 37$ pixels to cover the chip gap in both WFC3/UVIS filters, since we have essentially no later duplicate coverage of the fields with this camera.

We also could not obtain the set of images needed to achieve Nyquist sampling in the WFC3/IR F110W filter, due to the buffer-dump limits. However, we expect that every star that is detectable in the F110W image will also be detectable in either of the Nyquist-sampled F814W or F160W images.

All dithers are tabulated in Table 2. A concern at the time of the Phase-II preparation of the program was that the accuracy of the dithers needed to achieve simultaneous Nyquist sampling in the F160W exposures in parallel with the F475W exposures would be difficult to realize in practice. In fact, due to an unfortunate $\sqrt{2}$ error in the scale of the dithers, the first season of data (summer 2010) was not properly Nyquist-sampled. This error was corrected in time for all subsequent observations, however, and we find that the HST FGS is indeed able to perform the sub-pixel dithers to the level required. Figure 4 shows the dithers realized in F475W during the 2010 December-2011 February period of observation. This filter makes the strongest demands for dither accuracy of the three filters in which we will construct Nyquist-sampled images, but nearly all dithers fell within 0.15 pixel of the targeted offsets, which is sufficient to achieve the desired degree of sub-sampling (Lauer 1999).

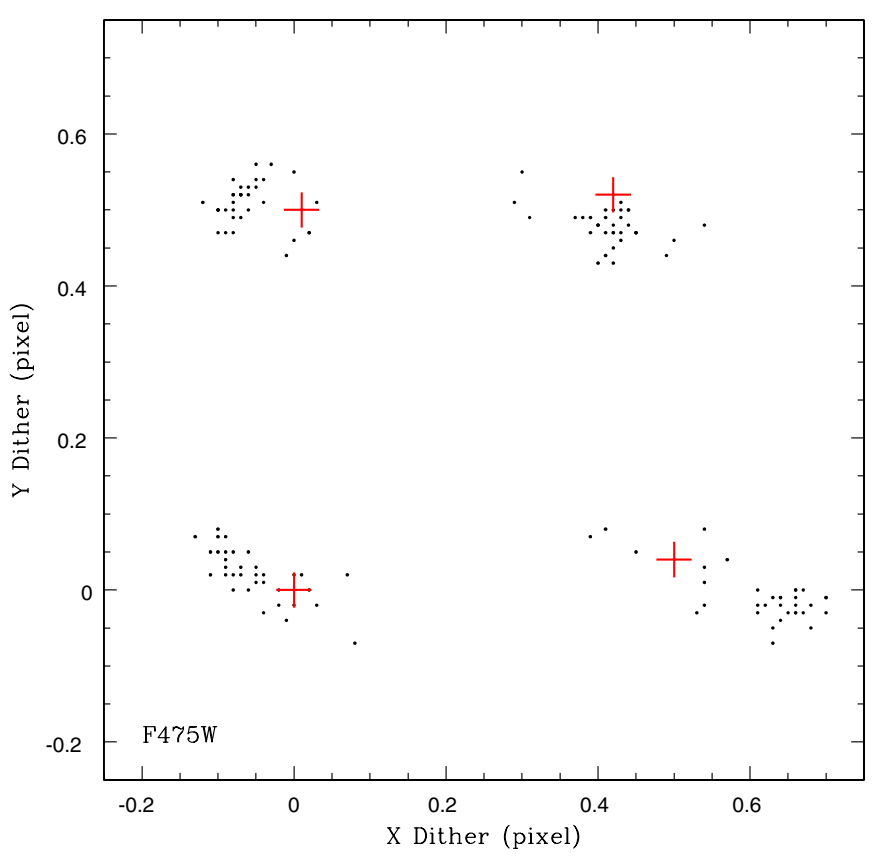

Figure 4. Sub-pixel dithers requested (red crosses) and achieved (points) are shown for ACS/WFC F475W images obtained during the winter 2010 observing season. Note that the requested dithers do not fall exactly at the optimal $2 \times 2$ 0.5 pixel locations, as the dither sequence operates on both the F475W and F160W filters simultaneously, and thus is a compromise between the needs of the two filters. The deviation of the actual dithers from the ideal interlace pattern is small enough that algorithms that can reconstruct Nyquist-sampled images from non-optimal dithers, such as that of Lauer (1999), should still work satisfactorily.

(A color version of this figure is available in the online journal.)

\subsection{Tiling Strategy}

We use the basic exposure sequence above (two orbits per pointing, one with WFC3/IR and one with WFC3/UVIS in primary, and ACS/WFC in parallel) as the foundation for a highly efficient tiling scheme.

The primary tiling is based on WFC3/IR, which has the smallest field of view (FOV) of all three imaging cameras. We arrange the WFC3/IR pointings in a $3 \times 6$ grid, with $<5^{\prime \prime}$ overlap among the WFC3/IR FOVs; due to distortion, the overlaps range from $\sim 1^{\prime \prime}$.5 to $\sim 5^{\prime \prime}$ for adjacent pointings. The WFC3/UVIS exposures use a small offset of 1.655 pixels in $\mathrm{X}$ and 2.96 pixels in $Y$ with respect to the standard UVIS-CENTER aperture, such that the same pointing center is maintained with respect to the IR-FIX aperture used to define the WFC3/IR pointings. The overlap between adjacent WFC3/UVIS exposures is $\sim 0.5-1^{\prime}$, due to the camera's larger FOV.

We cover the $3 \times 6$ "brick" with ACS by observing the two $3 \times$ 3 halves of the brick with two orientations, $180^{\circ}$ apart. Due to telescope constraints, observations in these two orientations are taken $\sim 6$ months apart, in two "seasons." In the first observing season, the observations produce a contiguous $3 \times 3$ half-brick of overlapping WFC3 observations, and an adjacent $3 \times 3$ half-brick of overlapping ACS observations. Due to the large ACS FOV, most points within the survey region have ACS data from two to four separate visits. In the second observing season, the telescope is rotated by $180^{\circ}$, and completes the $3 \times$ 3 WFC3 pointings on the half-brick covered previously with ACS. The switch in orientation leads the parallel ACS pointings to simultaneously cover the $3 \times 3 \mathrm{WFC} 3$ pointings taken during the previous orientation. 

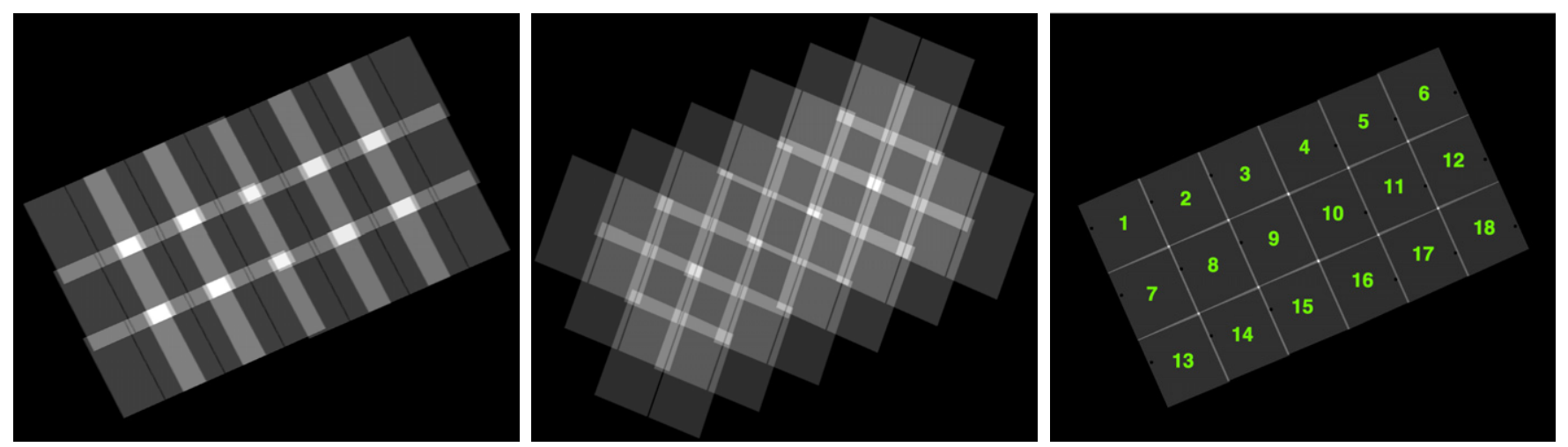

Figure 5. Maps of the relative exposure times across a brick for the WFC3/UVIS (left), ACS/WFC (middle), and WFC3/IR (right) tilings. The labeling of PHAT "fields" within a brick is shown superimposed on the WFC3/IR tiling. The left and right $3 \times 3$ half-bricks are taken with orientations $180^{\circ}$ apart, as can be seen by the relative position of the dark circle in the WFC3/IR exposure map. The WFC3/UVIS exposures are dithered to cover the chip gap. The WFC chip gap in ACS is not dithered within a single orbit, but is covered by overlapping exposures.

(A color version of this figure is available in the online journal.)

The resulting $3 \times 6$ brick has complete areal coverage in all three cameras, producing a contiguous area of $12^{\prime} \times 6.5$ in 36 orbits; exposure maps of the bricks are shown in Figure 5. Each pointing in the brick follows a consistent naming scheme, such that Field 1 is the pointing in the northeast corner of the brick, and Field 18 is the pointing in the southwest. The full naming scheme for each target position is of the form "M31-B\#\#-F\#\#-XXX," where "\#\#" represents a two-digit number; the number after $B$ indicates the brick number; the number after $\mathrm{F}$ indicates the field number within the brick; and the XXX indicates the camera on the WFC 3 or ACS instruments (UVIS, WFC, IR). Note that parallels are named according to the area they overlap, rather than for the location of the primary; thus, ACS images labeled as "B01-F04" overlap WFC3 images of the same name.

The default orientation of the brick was set to allow the observations to be maximally schedulable in both $180^{\circ}$ orientations. The optimal orientations had large numbers of schedulable days in the summer (peaking in July) and the winter (peaking in January). While a single orientation for the whole survey would be preferable for producing minimal overlaps between adjacent bricks, upon consultation with STScI it was decided to adopt two default orientations for the survey. This choice allowed slightly longer scheduling windows for the observations, reducing their impact on the scheduling of other programs. The 11 bricks closest to the center of the galaxy (Bricks 1-10, and Brick 12) are observed with ORIENT set to 69 (winter) or 249 (summer). The remainder of the bricks in the outer galaxy are observed with ORIENT set to 54 (winter) or 234 (summer). Each brick is assigned to a unique proposal ID number (PID). The approximate corners of the NIR footprint of the bricks and their PIDs are given in Table 3.

\subsection{Spectroscopy}

The PHAT imaging described here is being augmented with extensive spectroscopy. Individual stars in M31 are bright enough be observed spectroscopically, allowing us to measure the kinematics, metallicities, spectral types, and physical parameters of star clusters and massive main-sequence, AGB, and RGB stars.

The majority of PHAT spectroscopy to date has been carried out with DEIMOS on Keck $(R=6000, \sim 6400-9100 \AA)$, using a similar observing set-up as Gilbert et al. (2006), Kalirai et al. (2006a), and Guhathakurta et al. (2006). A total of 21 slitmasks covering much of the $H S T$ footprint have already been observed, and have produced $\sim 5000$ radial velocities accurate to $\sigma_{v}=5 \mathrm{~km} \mathrm{~s}^{-1}$ down to $I_{o}=22\left(\sim 2^{m}\right.$ fainter than the TRGB); preliminary reductions are described in Dorman et al. (2012). These spectra are being used primarily for kinematic decomposition of different M31 sub-populations. Future analyses will constrain stellar metallicities and spectral types from the $\mathrm{Ca}$ triplet and other absorption features. These data also can be used to estimate ionized gas metallicities from $\mathrm{H} \alpha /[\mathrm{N}$ II $] /\left[\mathrm{S}_{\mathrm{II}}\right]$ emission lines.

Additional spectroscopic programs are underway, with the goals of (1) measuring accurate $\mathrm{H}$ II region abundances using weak line methods; (2) measuring spectral types of hot stars using medium resolution spectroscopy at $<6000 \AA$; and (3) measuring the masses and metallicities of stellar clusters. When possible, these various spectroscopic programs also target serendipitous "interesting" sources from the PHAT survey, including PNe, AGN candidates, and X-ray counterparts. We are also extending the star cluster and PN survey of Caldwell et al. $(2009,2011)$ to PHAT targets, with more than 200 new spectra obtained thus far. These various spectroscopic programs will be described in future papers.

\section{DATA}

We now describe the present state of the PHAT observations and pipeline for image processing, stellar photometry, and astrometry. We also include assessments of the current data quality. Because this project is still actively acquiring data, we expect future data releases to incorporate ongoing improvements in data processing and in the resulting photometric catalogs. We note planned upgrades and processing revisions throughout this section, for cases where substantial development work is already underway.

\subsection{Observations}

Figure 1 and Table 1 summarize the observations discussed in this paper. We list bricks, their PIDs, and the range of dates over which data were taken for each orientation (i.e., the winter observations which populate WFC3 observations of the eastern halves of the bricks, and the summer observations which populate WFC3 observations of the western halves). Observations for the program began on 2010 July 12, and complete six-filter coverage is available for Bricks 1, 9, 15, 17, 
Table 3

Approximate Corners of Bricks ${ }^{\mathrm{a}}$

\begin{tabular}{|c|c|c|c|c|c|c|c|c|c|}
\hline Brick & PID & R.A. 1 & Decl. $_{1}$ & R.A.2 & Decl. $_{2}$ & R.A.3 & Decl.3 $_{3}$ & R.A. 4 & Decl. $_{4}$ \\
\hline 1 & 12058 & 10.87969 & 41.26532 & 10.63671 & 41.34631 & 10.57610 & 41.24387 & 10.81892 & 41.16290 \\
\hline 2 & 12073 & 11.12029 & 41.18549 & 10.87790 & 41.26699 & 10.81699 & 41.16468 & 11.05921 & 41.08321 \\
\hline 3 & 12109 & 10.95216 & 41.36298 & 10.70890 & 41.44413 & 10.64809 & 41.34173 & 10.89118 & 41.26061 \\
\hline 4 & 12107 & 11.19357 & 41.28269 & 10.95092 & 41.36434 & 10.88980 & 41.26207 & 11.13230 & 41.18044 \\
\hline 5 & 12074 & 11.04482 & 41.45377 & 10.80133 & 41.53511 & 10.74029 & 41.43276 & 10.98361 & 41.35144 \\
\hline 6 & 12105 & 11.28646 & 41.37401 & 11.04358 & 41.45586 & 10.98223 & 41.35363 & 11.22495 & 41.27182 \\
\hline 8 & 12075 & 11.37848 & 41.46474 & 11.13539 & 41.54679 & 11.07381 & 41.44462 & 11.31675 & 41.36260 \\
\hline 9 & 12057 & 11.22980 & 41.63532 & 10.98587 & 41.71706 & 10.92436 & 41.61481 & 11.16813 & 41.53310 \\
\hline 10 & 12111 & 11.47410 & 41.55495 & 11.23079 & 41.63719 & 11.16898 & 41.53508 & 11.41213 & 41.45287 \\
\hline 11 & 12115 & 11.28791 & 41.71003 & 11.02353 & 41.74143 & 10.99982 & 41.63066 & 11.26404 & 41.59933 \\
\hline 12 & 12071 & 11.54277 & 41.65341 & 11.29918 & 41.73581 & 11.23717 & 41.63373 & 11.48060 & 41.55137 \\
\hline 13 & 12114 & 11.31717 & 41.81950 & 11.05228 & 41.85054 & 11.02883 & 41.73975 & 11.29355 & 41.70886 \\
\hline 14 & 12072 & 11.57892 & 41.78777 & 11.31430 & 41.81941 & 11.29041 & 41.70867 & 11.55487 & 41.67718 \\
\hline 16 & 12106 & 11.63914 & 41.89310 & 11.37413 & 41.92490 & 11.35010 & 41.81416 & 11.61495 & 41.78253 \\
\hline 17 & 12059 & 11.48855 & 42.02423 & 11.22291 & 42.05568 & 11.19909 & 41.94492 & 11.46456 & 41.91363 \\
\hline 18 & 12108 & 11.75272 & 41.99292 & 11.48736 & 42.02497 & 11.46310 & 41.91427 & 11.72830 & 41.88238 \\
\hline 19 & 12110 & 11.62005 & 42.12072 & 11.35409 & 42.15246 & 11.33001 & 42.04174 & 11.59581 & 42.01015 \\
\hline 20 & 12112 & 11.88344 & 42.08995 & 11.61776 & 42.12230 & 11.59324 & 42.01163 & 11.85876 & 41.97944 \\
\hline 21 & 12055 & 11.68571 & 42.22514 & 11.41935 & 42.25704 & 11.39512 & 42.14633 & 11.66131 & 42.11460 \\
\hline 22 & 12076 & 11.95062 & 42.19503 & 11.68455 & 42.22754 & 11.65987 & 42.11690 & 11.92579 & 42.08456 \\
\hline 23 & 12070 & 11.86064 & 42.31678 & 11.59401 & 42.34909 & 11.56944 & 42.23843 & 11.83591 & 42.20629 \\
\hline
\end{tabular}

Note. ${ }^{\text {a }}$ All coordinates given in J2000. Corners are given for the approximate limits of the WFC3/IR coverage; WFC3/UVIS and ACS/WFC images will extend beyond these limits (see Figure 5).

and 21 as of fall 2011. Brick 23 is nearly complete, but two fields are being re-observed due to guiding failures (Section 4.1.1). Our primary scheduling priority is to finish bricks, and we therefore anticipate that observations of the current "half-bricks" $(2,5,8,12,14,16,18$, and 22) will be completed in the winter 2011/2012 observing season. Observations are currently scheduled for the first halves of Bricks 4 and 6, as well.

Figure 6 shows false-color images (generated by Zolt Levay of STScI) of Bricks 1, 9, 15, and 21, which were the first four bricks completed in the program. Figure 7 shows a small portion of a false-color image for Brick 9 and reveals the rich information that can be seen at full $H S T$ resolution. The coverage as of fall 2011 is shown in Figure 8, overlaid on a GALEX image.

\subsubsection{Known Problems}

In a data set this large, there are bound to be glitches in the observations. Here we briefly point out known issues with some of the observations.

There were several problems affecting observations of Brick 23 (PID GO-12070). The FGS failed to acquire guide stars during Visits 03 and 13, making data from these observations unusable. As a result, Brick 23 is currently missing high-quality WFC3 observations for Fields 03 and 13, and ACS observations for Field 16; the ACS parallels in Field 06 appear to be usable. Data will be taken for these fields in the winter 2011/2012 observing season. In addition, one WFC3/UVIS exposure for target M31-B23-F12-UVIS was not completely read off the HST recorder before being overwritten. As a result, half of one line is missing from the image.

There are also currently missing observations in Brick 16 (PID 12106). In one of our requested orientations, there is no guide star available for the pointing that covers Field 17 with WFC3 and Field 14 with ACS. We therefore have to observe these two regions with the other orientation and will have to point at each independently. The additional orbits needed for these observations have been "borrowed" from the interface between the two brick orientations, where fields in Brick 13 have a large degree of overlap with Brick 15. We anticipate that data will be taken for these fields in the winter 2011/2012 observing season.

An FGS guide star lock failure also affected one observation in Brick 1 (Visit 3, Orbit 1, of GO-12058 on 2010 December 17). This target was subsequently re-observed on 2011 January 3.

In addition to the above observational difficulties (which should be remedied in upcoming observations), visual inspection of the images shows that a small portion of them are slightly affected by a number of different artifacts, most of which are well-known features of previous $H S T$ imaging observations (see HLA ISR 2008-01 by M. Stankeiwics, S. Gonzaga, and B. Whitmore for ACS, and ISR WFC3 2011-16 by P. McCullough for WFC3/UVIS). Strongly overexposed stars in ACS/WFC and WFC3/UVIS, for example, exhibit "bleeding-charge" tails, which are common to all images obtained with CCDs. At the same time, bright stars also generate a number of scattered-light artifacts in ACS/WFC and WFC3/UVIS, such as weak out-offocus pupil reflections or "ghosts," which often manifest as pairs of highly elliptical rings of light, somewhat resembling a pair of reading spectacles. Bright stars also generate more diffuse or complex "flares" of light, known as "dragon's breath" to users of these instruments. Both the dragon's breath and the "spectacles" can occur at angles of a few arcminutes away from the bright stars, which may not be even present in the image. These artifacts should have minimal impact on our photometry, given that they affect only a trivial fraction of the survey area. Moreover, because our photometry uses local sky measurements, these additive effects usually have little impact on our photometry, beyond increased noise from the elevated background.

While scattered-light artifacts will be repeated in all images within a given exposure sequence, there are also transient events 

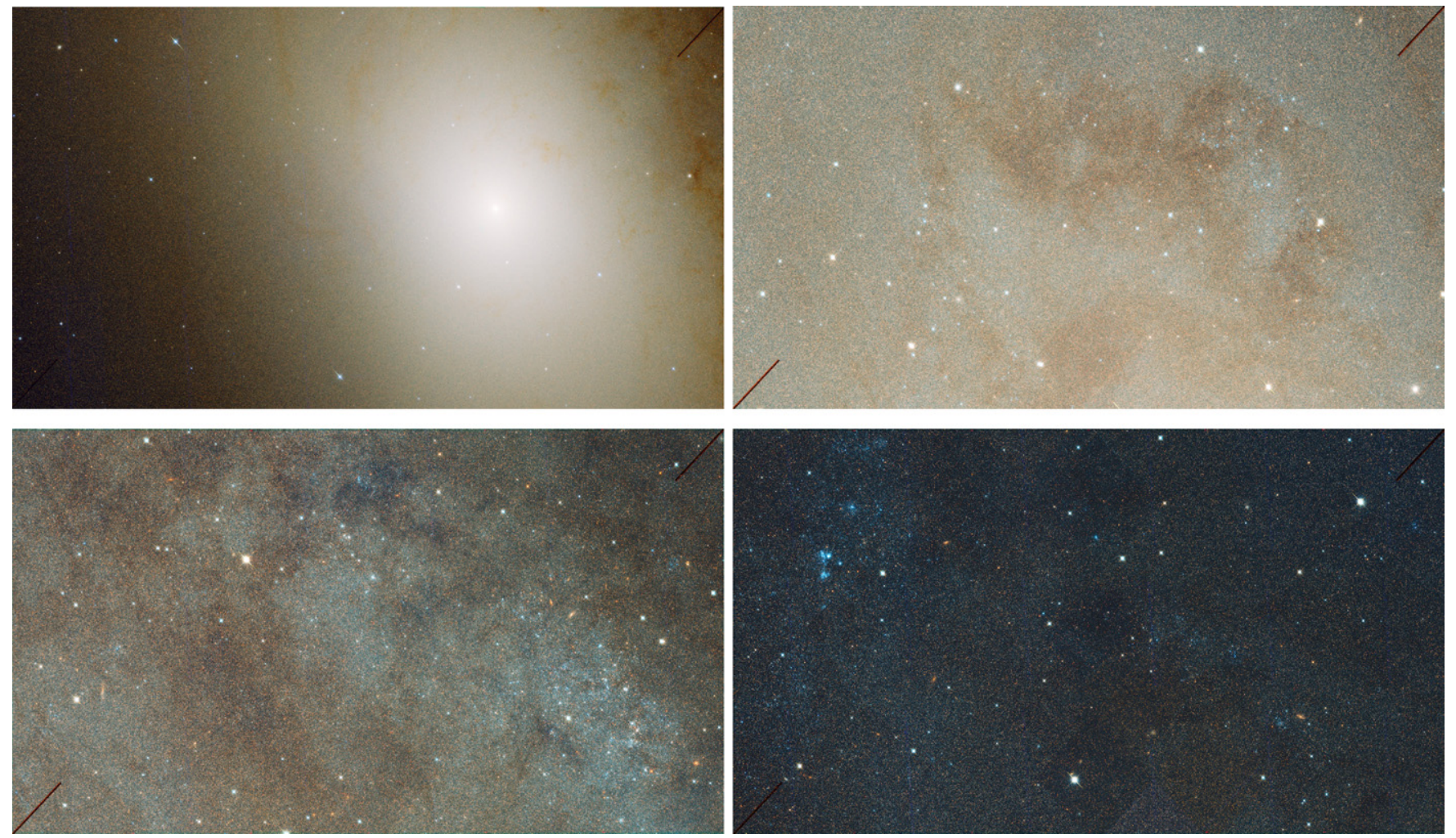

Figure 6. Four-color composite (F336W + F475W + F814W + F160W) images of Bricks 1, 9, 15, and 21 (upper left to lower right, respectively). The flux scaling is identical for Bricks 9, 15, and 21 to better show the radial change in the stellar density. Dark diagonal lines in the upper right and lower left corners are produced by the ACS/WFC chip gap (see Figure 5). Images were created by Zolt Levay (STScI).

(A color version of this figure is available in the online journal.)

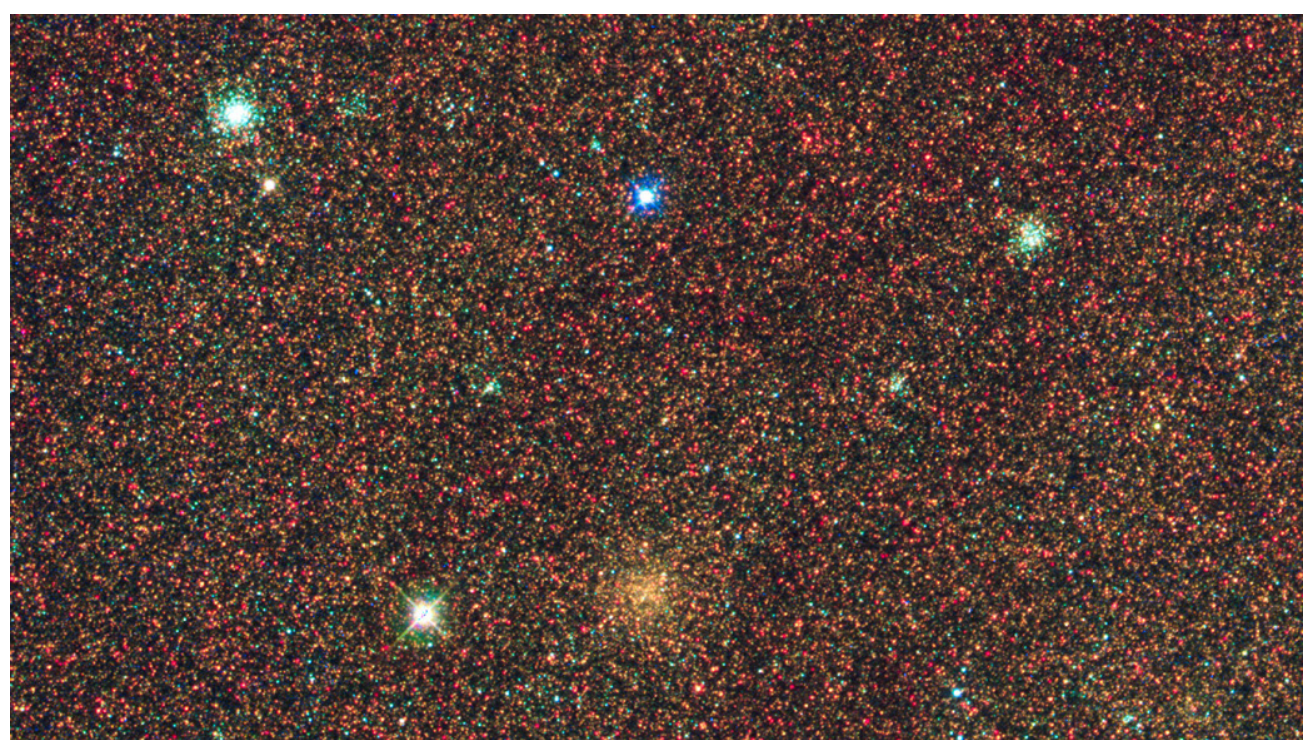

Figure 7. Close-up of a false-color image of Brick 9, showing the rich detail available at full HST resolution. Courtesy Zolt Levay (STScI).

(A color version of this figure is available in the online journal.)

that typically affect only one image in the sequence. Surprisingly common events are long trails due to "space debris" passing through the field during an exposure. In some cases the trail may affect both CCDs in ACS/WFC or WFC3/UVIS. These are most likely small particles in orbit around the Earth-in many cases the object generating the trail is clearly out of focus, but a point source will come into focus only when it is $\gtrsim 2 \times 10^{4} \mathrm{~km}$ away from the telescope. Again, we have done no special processing to remove these trails or the spurious sources that they may produce; such processing is unnecessary for ACS, where all but the faintest trails will be flagged as CRs, but additional image flagging could potentially be beneficial for WFC3/UVIS, where there are only two exposures per filter. The trails are typically extremely straight and uniform, and thus catalog-level contamination might be described with a fairly simple model. At the other end of the scale, we have also detected a few asteroids, which may be seen at different locations in several exposures within a sequence. 


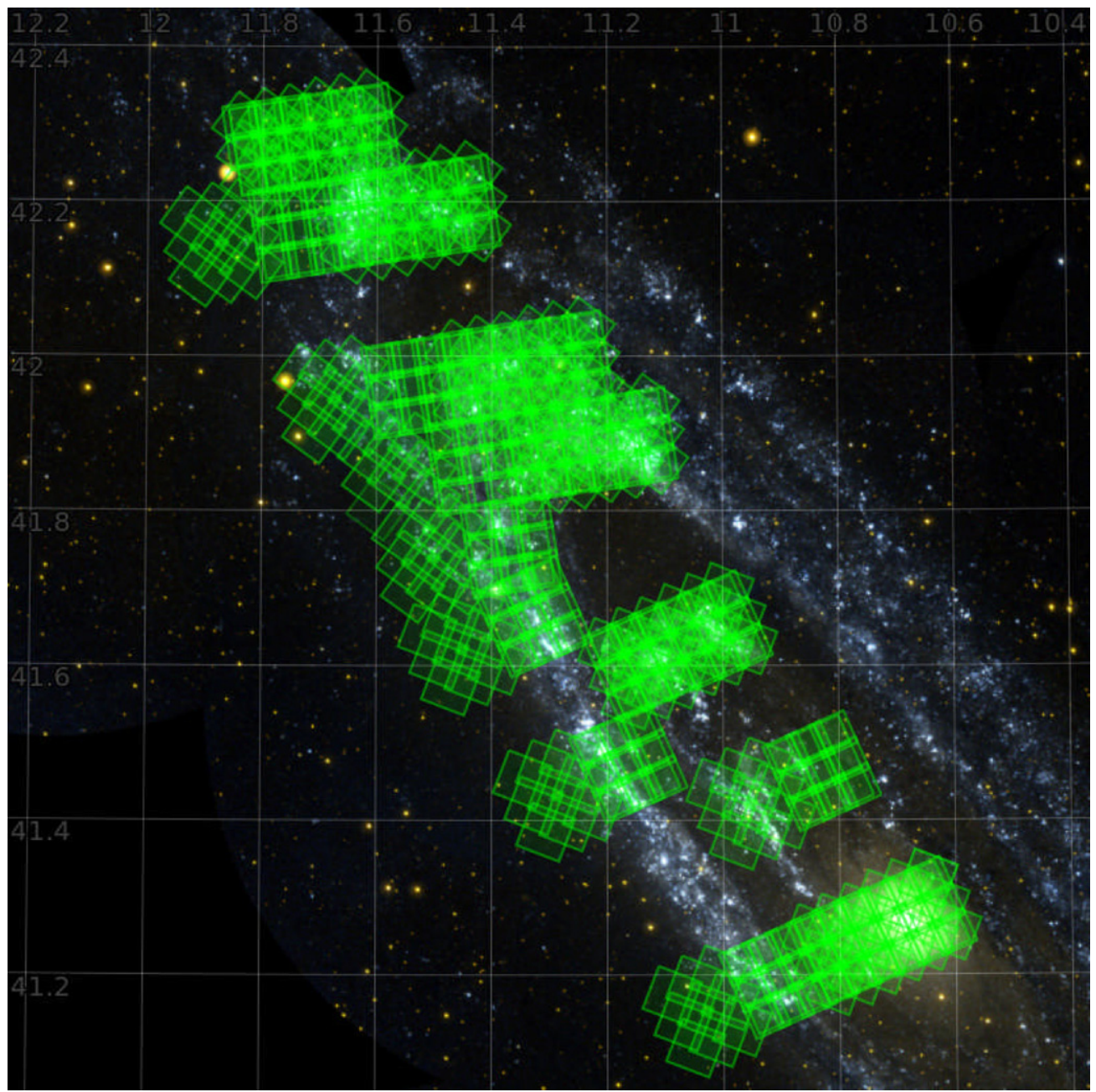

Figure 8. Current PHAT coverage, as of Fall 2011, superimposed on a GALEX FUV + NUV image. Bricks 1, 9, 15, 17, 21, and 23 are largely complete, as are the first halves of Bricks 2, 5, 8, 12,14, 16, 18, and 22. There are missing data in Brick 16 (Fields 14 and 17) and Brick 23 (Fields 13 and 16) that will be filled in during future observation cycles.

(A color version of this figure is available in the online journal.)

The other transient scattered-light artifact seen in our data is the illumination of $\sim 1 / 3$ of the WFC3/IR channel when pointed near a bright Earth limb. This effect has been described and characterized in Dalcanton et al. (2012), and is likewise thought to have very little impact on the photometry.

Several exposures exhibit strong CR "clusters," in which several dozen CR events form a tight cluster, superficially resembling a small star cluster. These events can be corrected with standard CR repair algorithms; however, it may be worth noting that most of the data of the image affected may be lost over the extent of the cluster.

Lastly, we note that errors in the transmission of the images to the ground in rare cases have resulted in the loss of a small number of pixels in a few images. The lost pixels typically occur in a small contiguous segment of a few hundred pixels within a single row on the CCD imagers (we have not experienced any lost data with WFC3/IR). In all cases, the lost data have been flagged in automatic reduction of the images, both in the dataquality image, and as anomalous values in the flattened image itself.

\subsection{Image Processing}

The first goal of our data processing is the production of a homogeneous catalog of six-band photometry for all of the stars detected in our survey area. Obtaining such a catalog requires well-calibrated images that are cleaned of CRs.

The ACS camera has been well studied and is well calibrated in general. However, the camera has had issues with the bias level since the replacement of key electronic components during Servicing Mission 4. For WFC3, the calibration has been steadily evolving over the course of our survey. These issues have required us to go beyond the standard pipeline image processing when bias-correcting, flat-fielding, and flagging CRs in our imaging data. We now describe the image processing and CR rejection for the ACS and WFC3 cameras.

$$
\text { 4.2.1. ACS }
$$

The ACS data were processed starting with the *.flt images from the $H S T$ archive. These images were flat fielded by the OPUS pipeline. As our data come in every 6 months, multiple versions of the OPUS pipeline were applied to different portions of our data. Depending on the observation date, versions 2010_2, 2010_3, and 2011_1e were used to generate the $*$.flt images. Since the ACS calibrations have been stable for several years, any changes are likely to be slight and are unlikely to affect the photometry.

Due to issues with the ACS bias level (ISR ACS 2011-05 by N. Grogin et al.), the pipeline-processed images 
suffer from several problems. The first major problem is that the ACS bias level shows a clear striping pattern, whose rowto-row fluctuations vary from image to image. These bias level variations are only apparent in images where the sky level was low; in such cases the row-to-row changes in the bias level have a larger amplitude than the typical sky noise. The second notable problem is that, in rows where the default pipeline bias subtraction was poor, the initial data-quality extensions from STScI flag a high percentage of pixels as being affected by CRs. We reset these CR-flagged pixels to allow us to determine the CR-affected pixels independently, after correcting for the bias problems.

To correct for the bias striping, we used a de-striping algorithm developed by Norman Grogin (csc2_kill.py, ISR ACS 2011-05). This algorithm attempts to correct images for striping, but is only helpful in cases where the bias striping can be accurately measured above the sky noise and where the bias-striping corrections are not inadvertently correcting for real structure in the background sky (say, in the bulge, where there are large gradients in the unresolved light). Therefore, after initially applying the de-striping algorithm, we then test the results to be sure that the changes to the image are at the level appropriate for bias-stripe correction. To do so, we plot histograms of the difference between the final and corrected pixel values in one CCD column. If this distribution has an amplitude and width characteristic of the bias striping as described in the ISR, we replace the original *.flt image with the de-striped version. Otherwise the exposure is deemed to be unaffected by the striping, and the original exposure is unchanged. Roughly $50 \%$ of the exposures were kept as is.

After bias striping is corrected (if needed), we flag CRs as follows. For our longer exposures, the exposure times are similar enough to allow for reliable rejections based on high outliers from the median of the image stack. Therefore, all exposures longer than $50 \mathrm{~s}$ were put through the PyRAF routines tweakshifts and multidrizzle, which flags CR-affected pixels using this median-image technique. We found that CR rejection was far too aggressive when the short "guard" exposures were included in the image stack. We therefore handle $\mathrm{CR}$ rejection in the short exposures independently. All ACS exposures shorter than $50 \mathrm{~s}$ are instead put through the IDL routine lacosmic (van Dokkum 2001), which uses Laplacian edge detection to flag pixels that show sharp edges associated with CRs, and which proved to be effective for removing obvious CRs from short single exposures. Once these steps are completed, the ACS data are ready to enter our photometry pipeline.

\subsection{2. $W F C 3$}

While our ACS imaging required only a few minor changes to the pipeline-processed images, WFC3 is a sufficiently new instrument that its entire calibration has been in a state of flux during our survey, making homogeneous calibration more of a challenge. Furthermore, our WFC3/UVIS data were obtained with just two exposures per filter, making CR-masking particularly difficult.

The WFC3 data were calibrated starting with the raw images, because the UVIS and IR flats and distortion calibrations are continuing to evolve. All of our data were processed in PyRAF with calwf3 in STSDAS version 3.12. We used a single set of flat fields for all the images used in this release (f110w_lpflt.fits, f160w_lpflt.fits, f275w_lpflt.fits, and f336w_lpflt.fits, with header dates of 2008 December 9, 2008 December 9, 2009 April 24, and 2009 March 31, respectively). For consistency, we also used a single set of distortion correction tables (210710_uvis_idc.fits, t20100519_ir_idc.fits, and u7n18501j_idc.fits for WFC3/UVIS, WFC3/IR, and $\mathrm{ACS}$, respectively). It is known, however, that the distortion has changed as a function of time, for ACS at least (ISR ACS 2007-08); in future releases, we will be solving for timedependent distortion corrections internal to the PHAT data set, as described below in Section 4.6. We anticipate updating all calibration images before each major rerun of the full data set.

After flat-fielding, we flagged CRs in the calibrated WFC3 images. The calibrated WFC3/IR images are essentially free of CRs, as expected due to the many non-destructive reads taken during data collection. However, the WFC3/UVIS images, which contain only two exposures in each filter, were plagued by CRs. We attempt to mitigate the $\mathrm{CR}$ effects by running all WFC3/UVIS exposures though the IDL routine lacosmic (van Dokkum 2001), as was done for the short ACS guard exposures. We also process the images through the PyRAF routines tweakshifts and multidrizzle using the minmed algorithm to flag CR-affected pixels.

Unfortunately, even after these techniques are applied, the WFC3/UVIS data still contain some obvious CRs. More aggressive $\mathrm{CR}$ rejection was found to eliminate central pixels of stars, and thus we cannot pursue more aggressive $\mathrm{CR}$ rejection at the image-processing level. Instead of risking degradation of photometry for real stars, we cull CR artifacts from our photometric catalogs, since they have poor fits to the PSF model and are anomalously "sharp." We are therefore able to cleanly remove CR-affected photometric measurements in our post-processing, as will be detailed in our description of photometry. Thus, while the residual CRs result in less attractive looking images, they do not affect our final photometry catalogs significantly. We plan to continue to experiment with ways to generate cleaner WFC3/ UVIS images as the survey continues.

After calibration, all WFC3 and ACS images are masked using the data-quality extensions of the $*$.flt images, which are created during image processing. The only modification was to accept data in the small IR blobs (data-quality flag 512), as described in Section 3.4. All images are then multiplied by the appropriate pixel area map to allow for accurate photometry to be performed on the original calibrated and undrizzled images.

\subsection{Measuring Resolved Stellar Photometry}

We measure stellar photometry on stacks of the final calibrated and CR flagged images. The PHAT images are extremely crowded, making PSF fitting the only viable technique for producing accurate photometry. This technique requires a wellmeasured PSF model, calibration of that model against aperture photometry, and an algorithm for fitting the PSF and local background to all sources.

All of our photometry was produced by the software package DOLPHOT 1.2 (Dolphin et al. 2002 and many unpublished updates). This software iteratively identifies peaks and uses the PSF models from TinyTim (Krist 1995; Hook et al. 2008) to simultaneously fit the sky and PSF to every peak within a stack of images, for multiple filters taken with a single camera. Minor corrections for differences between the PSF model and the true PSF in each exposure are calculated by DOLPHOT using neighbor-subtracted bright stars in the field, primarily to account for changes in the telescope focus. Since each exposure is fit simultaneously, the original HST PSF is left intact to provide 
Table 4

DOLPHOT Processing Parameters

\begin{tabular}{|c|c|c|c|c|}
\hline Description & Parameter & WFC3/UVIS & ACS/WFC & WFC3/IR \\
\hline Photometry aperture size & RAper & 8 & 8 & 5 \\
\hline Inner sky radius & RSky0 & 15 & 15 & 8 \\
\hline Outer sky radius & Rsky1 & 35 & 35 & 20 \\
\hline$\chi$-statistic aperture size & Rchi & 3.0 & 2.0 & 1.5 \\
\hline Photometry type & PSFPhot & 1 & 1 & 1 \\
\hline Fit sky? & FitSky & 3 & 3 & 3 \\
\hline Spacing for sky measurement & SkipSky & 2 & 2 & 2 \\
\hline Sigma clipping for sky & SkySig & 2.25 & 2.25 & 2.25 \\
\hline Second pass finding stars & SecondPass & 25 & 25 & 25 \\
\hline Searching algorithm & SearchMode & 1 & 1 & 1 \\
\hline Sigma detection threshold & SigFind & 3.0 & 3.0 & 3.0 \\
\hline Multiple for quick-and-dirty photometry & SigFindMult & 0.85 & 0.85 & 0.85 \\
\hline Sigma output threshold & SigFinal & 3.5 & 3.5 & 3.5 \\
\hline Maximum iterations & MaxIT & 25 & 25 & 25 \\
\hline Noise multiple in imgadd & NoiseMult & 0.10 & 0.10 & 0.10 \\
\hline Fraction of saturate limit & FSat & 0.999 & 0.999 & 0.999 \\
\hline Use saturated cores? & FlagMask & 4 & 4 & 4 \\
\hline Find/make aperture corrections? & ApCor & 1 & 1 & 1 \\
\hline Force type $1 / 2 ?$ & Force1 & 1 & 1 & 1 \\
\hline Align images? & Align & 2 & 2 & 2 \\
\hline Allow cross terms in alignment? & Rotate & 1 & 1 & 1 \\
\hline Centroid box size & RCentroid & 1 & 1 & 1 \\
\hline Search step for position iterations & PosStep & 0.1 & 0.1 & 0.1 \\
\hline Maximum single step in position iterations & dPosMax & 2.5 & 2.5 & 2.5 \\
\hline Minimum separation for two stars for cleaning & RCombine & 1.415 & 1.415 & 1.415 \\
\hline PSF size & RPSF & 10 & 10 & 10 \\
\hline Minimum $\mathrm{S} / \mathrm{N}$ for PSF parameter fits & SigPSF & 3.0 & 3.0 & 3.0 \\
\hline Make PSF residual image? & PSFres & 1 & 1 & 1 \\
\hline Coordinate offset & Psfoff & 0.0 & 0.0 & 0.0 \\
\hline WFC3 PSF library & UseWFC3 & 1 & $\cdots$ & 1 \\
\hline ACS PSF library & UseACS & $\cdots$ & 1 & $\cdots$ \\
\hline
\end{tabular}

the highest possible quality PSF fits, making these corrections as small as possible, typically of order 1\%-5\% (see Section 4.3.1).

The exact processing steps employed by DOLPHOT are described in detail in Dolphin (2000). To summarize, DOLPHOT uses the following steps to determine the star list and stellar photometry from a stack of aligned images for a single camera.

1. An iterative search for stars in the image stack.

2. Iterative PSF-fitting photometry of individual stars, using images with stellar neighbors subtracted.

3. PSF adjustment using bright, relatively isolated stars on neighbor-subtracted images.

4. A second round of iterative PSF-fitting photometry on neighbor-subtracted images, using the refined PSF.

5. Calculation and application of aperture corrections derived from bright, relatively isolated stars on neighbor-subtracted images.

6. Computation of individual VEGAMAG instrumental magnitudes and uncertainties.

7. Application of charge transfer efficiency (CTE) corrections to the ACS magnitudes; WFC3/UVIS CTE corrections will be included when they become available from STScI.

8. Computation of combined per-filter VEGAMAG magnitudes from all measurements that are unsaturated and contain enough valid pixels near the core (i.e., do not fall on a CR or bad column).

Because DOLPHOT works on the uncombined image stack, precise alignment of the images is crucial for producing reliable PSF photometry. Any errors in image alignment would lead directly to systematic photometric errors, due to the PSF peak being misaligned with those of the stars. DOLPHOT solves for the relative alignment of images by matching bright stars, starting from an initial estimate of the shifts provided by the user. The distribution of alignment values taken from the matched stars typically shows a clear central peak, with a width of order $\sim 0.1$ pixels for the star-to-star alignments within a single image. The resulting image-to-image alignment is therefore good to $\ll 0.1$ pixels. The PHAT processing pipeline flags cases where the distribution of alignment values lacks a central peak or has a width of a pixel or more; these rare cases are then subject to further by-hand intervention.

We adopted DOLPHOT parameters (Table 4) that would give high completeness in highly crowded regions and minimize systematics in each camera, and that also could be used for fields with a wide range of stellar density (thus yielding homogeneous photometry from the bulge to the outer disk). We selected these initial parameters based on past experience with stellar population surveys (Dalcanton et al. 2009, 2012), and on a coarse sampling of parameter space. The resulting parameters appear to have accomplished our goals, as they yield reliable results throughout the survey area according to our fake star tests (Section 4.7 below). However, given that we have identified a few issues affecting photometry at the few percent level (described below), we expect to optimize the processing parameters further as the survey progresses. As such, we are currently experimenting with a larger grid of potential DOLPHOT parameter sets to quantitatively determine the optimal set for precision and completeness. These parameters will likely be updated in our 
next data release, with details of any changes being described in subsequent papers and in supporting materials provided with the data distribution via the Multi-Mission Archive at Space Telescope (MAST).

The key parameters for producing high-quality photometry have proved to be Rchi and Fitsky. Rchi is the radius inside of which the centroid of the PSF is fit. This value is set to be quite small (about one full width at half-maximum of the PSF) to avoid it being affected by poorly subtracted neighbors. Subsequent star matching between cameras and astrometric alignment also uses this same value of Rchi. Fitsky sets how the local background level is measured; we have adopted Fitsky $=3$, which forces simultaneous fits to the PSF and the background level inside of the fitting radius. This strategy is optimal for very crowded fields, but is also computationally expensive. For this first pass through our data we conservatively applied this algorithm for sky measurement.

Once the PSF-fitted magnitudes of all stars have been measured, the values are corrected to a 0 .'.5 aperture, equivalent to using an aperture correction measured using neighbor-subtracted bright stars on the image (see Section 4.3.2).

The ACS/WFC and WFC3/UVIS images both suffer from CTE problems, such that not all charge is read out of a pixel, leaving residual charge that is instead read out in subsequent rows. This effect leads to a small bleed trail of charge that is most severe for low electron levels and for rows near the beginning of the readout (i.e., near the chip gap for the ACS/WFC and WFC3/UVIS packages). The trail of charge is made up of flux that should have been included in the main body of the star, and thus photometry of stars affected by CTE will be systematically faint, and positions of the affected stars will be slightly shifted in the $Y$-direction.

Luckily, CTE is sufficiently well behaved that it is possible to derive reasonable corrections for its effects. The fluxes for ACS measurements are corrected for CTE using the prescription of Chiaberge (ISR ACS 2009-01). Typical corrections range from 0 to $0.2 \mathrm{mag}$ in Brick 01 where the background is high (hundreds of counts per pixel) to $0-0.5$ mag in Brick 21 where the background is low (tens of counts per pixel). $Y$-coordinates closer to the chip gap have larger corrections, as do fainter stars, which leads to the broad range in correction values.

We have also examined the possibility of using the imagebased CTE corrections of Anderson \& Bedin (ISR ACS 2010-03), which correct images for CTE loss prior to running our pipeline. Unfortunately, the procedure in its current form has the characteristic that all peaks, including noise spikes in the background, are treated as stars. This has the result of magnifying background noise peaks and CRs above our detection threshold, creating enormous numbers of very faint false detections, most notably near the chip gap. Given this complication, we have chosen to retain the catalog-based CTE corrections for their superior noise properties at faint magnitudes.

Currently, there are no CTE corrections available for WFC3/UVIS. Given that CTE is clearly evident in the WFC3/UVIS images (manifesting as obvious trails in $Y$ for sources near the chip gap), our WFC3/UVIS photometry is undoubtedly biased to fainter fluxes for sources close to the chip gap. We unfortunately cannot constrain the size of the effect directly with our data, since our tiling scheme is such that overlapping WFC3/UVIS observations leave stars at comparable $Y$ chip positions (see Figure 5). We will be incorporating WFC3/UVIS CTE corrections into our photometry as they become available.
Finally, the fluxes are transformed to infinite aperture and converted to Vega magnitudes using the zero points dated 2008 July 15 in the ACS Users Handbook and ISRs WFC3 2009-30 and WFC3 2009-31 by J. Kalirai et al. for WFC3. These zero points will be updated should new values be published before our next run through the full data set.

The photometry for this first pass through the PHAT survey data was performed on the data from each field and each camera separately. Therefore, any depth that may be gained from overlapping data from neighboring fields is not included in the current photometry. This added depth will be of only modest benefit in the crowding-limited portions of the survey, but will be significant in other portions. We anticipate that our second data release will contain simultaneous photometry on full image stacks, for all cameras.

Before discussing the production of the final photometric catalogs (Section 4.4), we briefly discuss the amplitudes of the PSF and aperture corrections.

\subsubsection{Amplitude of PSF Corrections}

The TinyTim PSF models used for PSF photometry are unfortunately not perfect. For ACS, they are known to vary with time (see Figures 8 and 9 in ISR ACS 2006-01 by J. Anderson and I. King) and to have small errors with position (e.g., Jee et al. 2007). For WFC3, the errors are likely to be even larger, because no post-flight updates to the TinyTim models have been implemented at this time.

Due to these temporal variations in the PSF, as well as possible systematic errors in the TinyTim PSFs themselves, DOLPHOT computes a PSF adjustment image that is added to each PSF. This calculation is done midway through the iterative photometry process, once a reasonably final star list has been reached. The process involves analysis of residuals near bright, relatively isolated stars in images in which all detected stars have been subtracted. Although an entire difference image (actual minus TinyTim model) is calculated and applied to the photometry, only the value of this image in the central pixel is reported. A positive value indicates that the data are sharper than the model; a negative value indicates the reverse.

Figure 9 shows the adopted PSF correction for the central pixel, for every image taken prior to Fall of 2011, with the exception of the short "guard" exposures in F475W and F814W. The amplitude of the typical PSF correction is relatively small $(<0.05$ in the UV, and $<0.01$ in the optical and NIR), and has no strong dependence on the local stellar density (i.e., Brick number) or the time of observation (not shown). Table 5 lists the median PSF correction and the semi-interquartile width, as well as the number of stars used to calculate the PSF correction. The scatter in the measured corrections increases for the bluer cameras, most likely due to the smaller number of high signalto-noise $(\mathrm{S} / \mathrm{N})$ sources in the WFC3/UVIS images.

The sign and amplitude of the central pixel PSF corrections are a first-order indication of the errors in the PSF model. For the WFC3/UVIS data, the PSF corrections are substantial and are consistently negative, indicating the current TinyTim model for the WFC3/UVIS PSF is slightly broader than the true data (i.e., the PSF correction must put extra flux back into the central pixel). The PSF corrections for ACS and WFC3/IR are a factor of three smaller, suggesting that the TinyTim models for these cameras are in better agreement with the data, at least within the very core of the PSF. The agreement outside the central pixel, however, is unconstrained by these initial tests. 


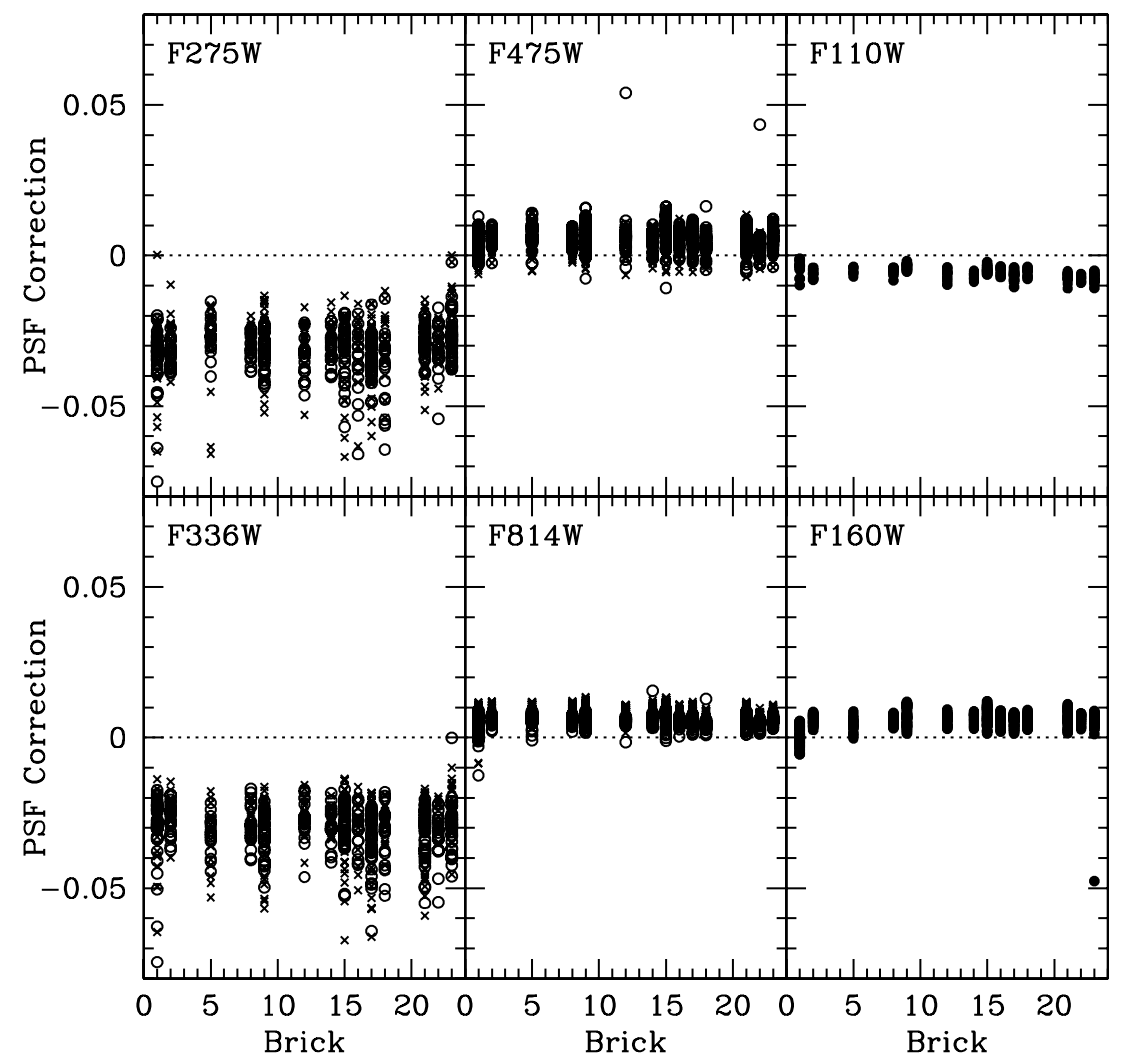

Figure 9. PSF corrections in magnitudes for all images in the survey to date, excluding the short guard exposures in F475W and F814W. For the WFC3/UVIS and ACS/WFC data, crosses and open circles indicate the corrections for chips 1 and 2, respectively. Median values are given in Table 5. The PSF corrections show no dependence on time or local stellar density.

Table 5

PSF and Aperture Corrections

\begin{tabular}{lcccrcc}
\hline \hline Camera & Filter & Chip & $n_{\text {psf }}$ & \multicolumn{1}{c}{ PSF Correction } & $n_{\text {ap }}$ & Aperture Correction \\
\hline WFC3/UVIS & F275W & 1 & 102 & $-0.0271 \pm 0.0047$ & 147 & $0.0584 \pm 0.0176$ \\
WFC3/UVIS & F275W & 2 & 93 & $-0.0307 \pm 0.0044$ & 125 & $0.0561 \pm 0.0167$ \\
WFC3/UVIS & F336W & 1 & 115 & $-0.0267 \pm 0.0049$ & 199 & $0.0188 \pm 0.0154$ \\
WFC3/UVIS & F336W & 2 & 111 & $-0.0304 \pm 0.0048$ & 199 & $0.0187 \pm 0.0142$ \\
ACS/WFC & F475W & 1 & 204 & $0.0051 \pm 0.0027$ & 198 & $0.0048 \pm 0.0082$ \\
ACS/WFC & F475W & 2 & 202 & $0.0064 \pm 0.0021$ & 198 & $0.0059 \pm 0.0083$ \\
ACS/WFC & F814W & 1 & 231 & $0.0091 \pm 0.0013$ & 200 & $0.0159 \pm 0.0057$ \\
ACS/WFC & F814W & 2 & 233 & $0.0057 \pm 0.0010$ & 200 & $0.0161 \pm 0.0053$ \\
WFC3/IR & F110W & & 241 & $-0.0059 \pm 0.0014$ & 199 & $0.0573 \pm 0.0057$ \\
WFC3/IR & F160W & & 241 & $0.0062 \pm 0.0015$ & 200 & $0.0784 \pm 0.0064$ \\
\hline
\end{tabular}

Notes. $n_{\mathrm{psf}}$ and $n_{\text {ap }}$ are the median numbers of stars used to calculate the PSF and aperture corrections, respectively. PSF and aperture corrections are listed as the median \pm the semi-interquartile range. All statistics have excluded data in Brick 1. The statistics for the short guard exposures in ACS have also been excluded.

\subsubsection{Amplitude of Aperture Corrections}

To calculate a star's magnitude, DOLPHOT adjusts the flux in the corrected TinyTim PSF to minimize residuals in the image stack. The model PSFs are scaled to unity within an aperture of $3^{\prime \prime}$ in radius, such that the internal magnitudes within DOLPHOT are calibrated as if the zero points were for $3^{\prime \prime}$ apertures. However, especially in crowded fields, we find it more practical to compute aperture corrections within a smaller aperture of 0.'5 in radius, and then apply encircled energy corrections to calibrate our photometry using the published zero points (which are for infinite aperture). DOLPHOT therefore corrects its internal PSF magnitude to an aperture magnitude calculated within a 0.5 radius. DOLPHOT calculates these aperture corrections by identifying $\sim 200$ bright, reasonably isolated stars in each image, computing aperture photometry on the neighbor-subtracted residual image, and then measuring the differences between these aperture magnitudes and the stars' PSF magnitudes. These measurements are then combined in a weighted average with outlier rejection to derive a single aperture correction which is applied to the PSF magnitudes of all stars in the image, for a given filter.

Figure 10 shows the aperture corrections for every image taken prior to Fall of 2011, excluding the short "guard" exposures in $\mathrm{F} 475 \mathrm{~W}$ and $\mathrm{F} 814 \mathrm{~W}$; Table 5 lists the median aperture correction and the semi-interquartile width for each filter, as well as the median number of stars used to calculate the aperture correction. The number of stars is typically high $(\sim 200)$ 


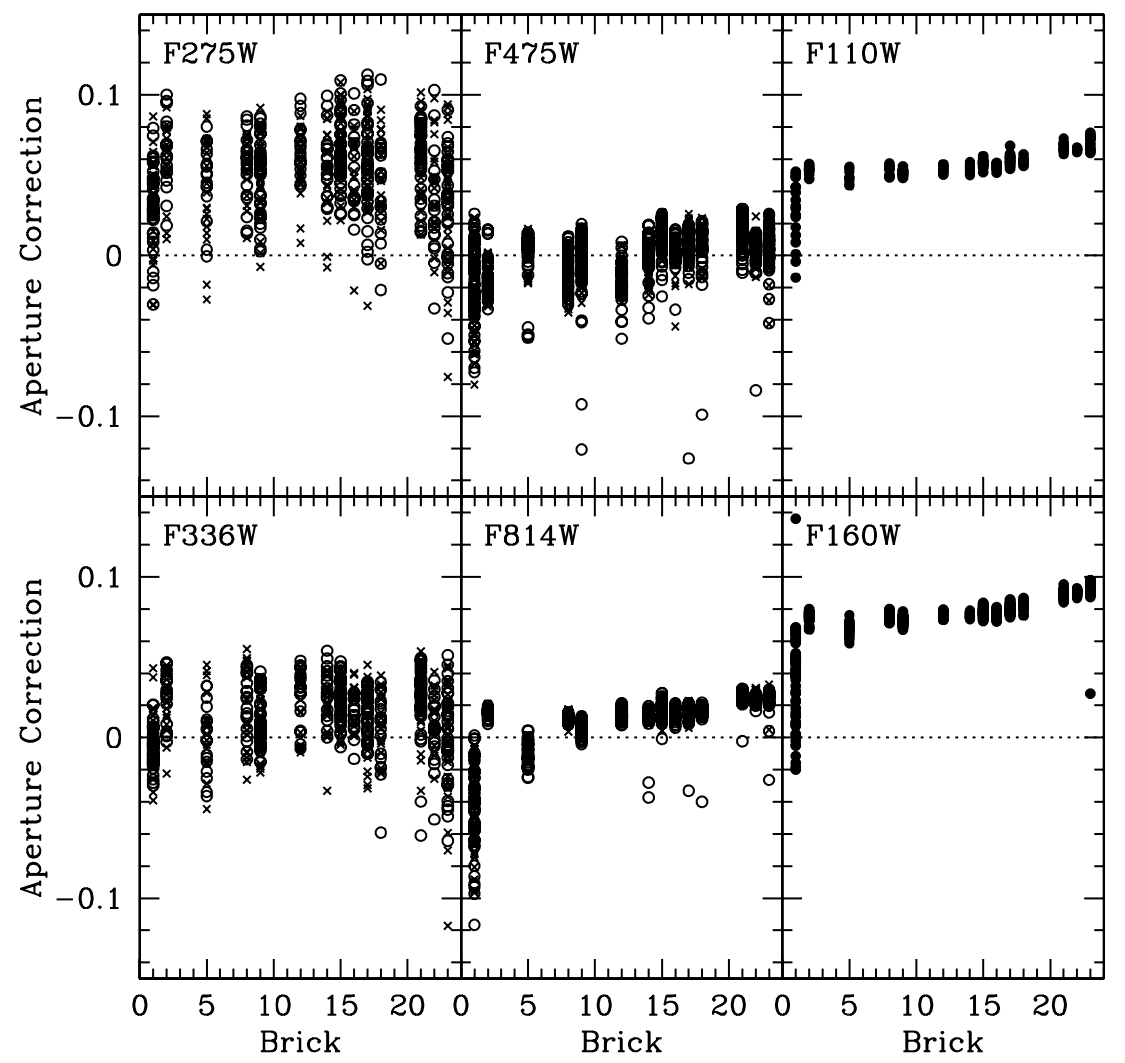

Figure 10. Aperture corrections in magnitudes for all images in the survey to date, excluding the short guard exposures in F475W and F814W. For the WFC3/UVIS and ACS/WFC data, crosses and open circles indicate the corrections for chips 1 and 2, respectively. The aperture corrections vary with brick number, due to variations in the local stellar density, which decreases for increasing brick number, and for even-numbered bricks. The scatter tends to be systematically larger for bluer filters. The scatter is largest in Brick 1, which has the largest range of stellar densities internal to the brick, due to the presence of the center of the bulge in Field 10. Median values are given in Table 5, excluding both the guard exposures and images from Brick 1.

for the crowded optical and NIR data. The UV fields, however, frequently suffer from a paucity of bright sources, leading to much smaller numbers of suitable stars and larger image-toimage scatter; this problem is most severe in Bricks 22 and 23 in the outer disk, and Brick 1 in the bulge. The Brick 1 bulge fields also suffer from reduced numbers of suitable stars in the optical and NIR, though not to the same degree as in the UV. The image-to-image scatter tends to be systematically larger for bluer filters, due to the lower number of high $\mathrm{S} / \mathrm{N}$ stars.

Table 5 shows that the mean aperture correction for a given filter is small $(<0.06 \mathrm{mag}$ for all but F160W), and has a typical image-to-image scatter of less than $0.02 \mathrm{mag}$ across the entire data set. However, inspection of Figure 10 indicates that the aperture corrections do have a modest systematic variation with brick number. We found no time dependence in the amplitude of the aperture correction, suggesting that the observed variation with brick number is likely driven by the systematic decrease in the stellar density and/or background level with increasing radius, since larger brick numbers and even brick numbers have larger projected radii. The effect is most noticeable in Brick 1 , which has the largest range of stellar densities internal to the brick, due to the presence of the center of the bulge in Field 10.

In contrast to what is seen in Figure 10, the aperture correction should in principle be a constant that solely corrects for the difference in the encircled energy between 0.5 and $3^{\prime \prime}$, assuming perfect PSF models and measurements. The fact that the aperture correction depends somewhat on the local level of crowding indicates that at least one of the key assumptions has been violated. In other words, the data in Figure 10 suggest that there is a slight bias in the measurement of the aperture correction calculated for bright stars, which may result from some combination of errors in the PSF model, background-dependent biases in the initial PSF magnitude, or subtle problems in constructing the empirical PSF used to measure the aperture correction. As such, it is not clear whether the observed crowding dependence in the aperture correction is introducing systematic errors or correcting for them.

At the present time, the $\sim 0.02$ mag scatter must be included in the error budget of the resulting photometry, with the understanding that there may be small systematic offsets in photometry measured in different regions of M31, particularly in Brick 1 for the NIR. However, the systematic variations in the aperture correction are typically smaller than empirical systematic errors derived from repeat measurements (shown below in Figure 18), suggesting the aperture corrections are not dominating the systematic error budget. They are also much smaller than the typical random uncertainties due to photon counting (Figure 13, below). We are currently investigating the origin of this bias, and expect to have it and other small systematic uncertainties resolved before the second data release.

\subsection{Producing Photometric Catalogs}

The lowest level data products produced by DOLPHOT are "*.phot" files, which include the photometry for every detected source (both optimized for the entire image stack, as well as for each individual image), along with several metrics for the quality of each photometric measurement. These catalogs offer 
the greatest amount of information, but are frequently much larger than needed for most applications. We therefore generate additional smaller catalogs restricted only to stellar sources with good photometric quality.

The metrics we have found most useful for generating high-completeness, low-contamination catalogs are measures of the "sharpness" and "crowding," which are quantified by DOLPHOT and returned as the parameters "sharp" and "crowd" for each star. Sharpness quantifies the degree to which an object is more or less centrally peaked than the PSF (see Equation (14) of Dolphin 2000). Very centrally peaked sources (positive sharpness) are likely CRs, as they do not have the wings associated with true point sources. Very diffuse sources (negative sharpness) are likely background galaxies or unresolved groups of stars. Crowding quantifies the degree to which the photometry of the source may have been affected by neighboring sources. The value for crowd is determined by calculating the difference between the magnitude measured for the star before and after the subtraction of neighboring sources. Large numbers of bright neighboring sources therefore lead to a large value for the crowding parameter.

Using these metrics, we cull lower-quality measurements from our catalogs to produce CMDs showing well-defined features. The cuts were done at two levels for each camera, generating two catalogs with different restrictions on data quality. The first cuts the photometry at the most basic level to ensure that all stars in the catalog have a high $\mathrm{S} / \mathrm{N}$ detection in at least one filter. The resulting "st" catalog contains only objects that either were detected with $\mathrm{S} / \mathrm{N}>4$ in both filters or were detected in one filter at $\mathrm{S} / \mathrm{N}>4$ with $\operatorname{sharp}^{2}<0.1$; the additional sharpness cut is used for the one-filter detections to suppress CRs. These cuts admit a very high percentage of all measurements but tend to remove any residual CRs from the catalogs.

The second cuts are designed to produce sharp-featured CMDs with well-measured colors, by removing highly crowded stars, stars with poor PSF fits and stars with very low S/N in either filter. These cuts were made by keeping only measurements with $\mathrm{S} / \mathrm{N}>4$ in both filters for a given camera, crowd ${ }_{1}+$ crowd $_{2}<1.0(0.48$ for WFC3/IR $)$, and $\left(\operatorname{sharp}_{1}+\operatorname{sharp}_{2}\right)^{2}<$ 0.075 ( 0.12 for WFC3/IR). These cuts were determined by experimenting with a series of possible cuts, looking at the CMD of the rejected objects, and choosing the cuts that removed the largest number of stars without generating strong features in CMDs made from the rejected stars. These gst ("good star") catalogs contain only stars with the highest quality photometry, but suffer from more incompleteness in crowded regions, such as dense stellar clusters. They are also limited in depth by the requirement that stars have good $\mathrm{S} / \mathrm{N}$ in both filters for a given camera. In cases where the two filters have very different depths for typical stellar colors (such as for F275W and F336W), the gst catalogs will be missing many high-quality detections in the deeper filter. Thus, when combining data from across cameras (say, when generating a F336W-F475W CMD), it may be preferable to revert to the st catalogs to avoid this restriction, but then to apply joint crowding cuts on the detections from the different cameras.

For PHAT data releases, the st and gst photometry catalogs are packaged into binary FITS tables, and distributed via MAST.

\subsection{Artificial Star Tests}

We assess the completeness and uncertainty as a function of color and magnitude of the st and gst catalogs using extensive artificial ("fake") star tests. We insert a series of $10^{5}$ fake stars into each field with the appropriate PSF, for each camera independently. This strategy produces 5.4 million fake star tests per brick. Each fake star is added individually to the image stack (i.e., one artificial star at a time), and the photometry is rerun in a sub-region around the star to determine if the star was found, and if so, to determine what its measured magnitude was; this method is efficient, and does not run the risk of artificially changing the local crowding. The measured properties of the fake stars are then put through our quality cuts to determine which stars would be included in the final st and gst catalogs.

The properties of the fake stars are chosen such that $50 \%$ of the stars randomly sample the entire observed range of color and magnitude, and 50\% sample the actual color-magnitude distribution measured for the individual field. Spatially, the stars are distributed randomly throughout each field. While they therefore will broadly sample the photometric quality of the field, they will not necessarily be representative of the photometry in stellar clusters, which have higher than average local densities, but which cover only a tiny fraction of the area.

Taking a coarse grid in color and magnitude, we calculate the completeness in each box of the grid and interpolate to find the $50 \%$ completeness points. The limiting magnitude in the red filter is then the $50 \%$ completeness magnitude in the red band, measured at a color that is blue enough to ensure that nondetection in the red filter was the cause for low completeness (i.e., blueward of the corner at the faint end of the CMD). The blue band limit is then the $50 \%$ completeness magnitude in the blue band, measured at a color that is red enough to ensure that non-detection in the blue filter was the cause for low completeness (redward of the corner at the faint end of the CMD). These 50\% completeness values are then tabulated as our magnitude limits in each field with the current processing version.

\subsubsection{Survey Depth}

Based on the $50 \%$ completeness limits, the faintest limiting magnitudes in the PHAT survey are found to be roughly 25.1, 24.8, 27.9, 27.1, 25.0, and 24.0 in the F275W, F336W, F475W, F814W, F110W, and F160W filters, respectively. For solar-metallicity main-sequence stars, these depths correspond roughly to $2.6 M_{\odot}, 2.8 M_{\odot}, 1.5 M_{\odot}, 1.5 M_{\odot}, 3.5 M_{\odot}$, and $6 M_{\odot}$, respectively, for zero foreground extinction. However, these depths are a strong function of local stellar density for the optical and NIR, as we now show.

\subsubsection{Crowding and Depth versus Radius}

For isolated stars, the depth of photometry is a constant set by the uncertainty in inferring a star's flux from a limited number of photons. At the surface density and distance of M31, however, one cannot assume that stars are isolated, or that their primary photometric uncertainties are due to photon counting. In M31's disk, the number of stars that fall above the limiting magnitude is sufficiently high that the PSFs of the individual stars will overlap. While crowdedfield photometry can compensate somewhat when detecting and measuring photometry for partially overlapping stars, at some point the level of overlap is sufficiently large that faint stars are completely undetectable. The crowding also adds additional photometric uncertainties to the detected stars, since the reported photometry for a single star now depends on subtracting flux 


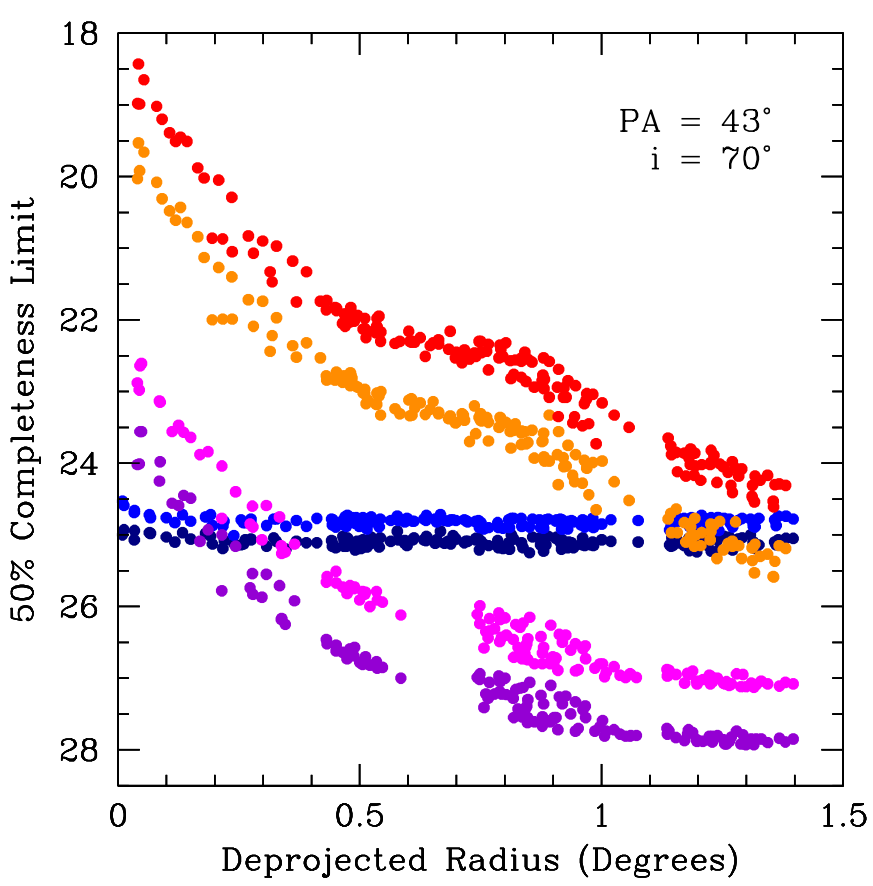

Figure 11. Magnitude of the 50\% completeness limit, plotted as a function of radius for the high photometric quality gst catalog detections, for all fields observed with the WFC3/UVIS (horizontal blue points), ACS/WFC (lowest two purple sequences), and WFC3/IR (highest red sequences) cameras. The two filters in each camera are color coded as in Figure 12. The UV observations are photon limited across the disk, with the exception of the very innermost regions of the central bulge field, and therefore have an essentially constant depth across the survey area. The optical observations are crowding limited over most of the disk, and thus the depth of the ACS observations is a strong function of radius. The optical data do become photon limited at a projected radius of $\sim 0.9$, beyond which the depth of the observations becomes constant. The NIR observations are crowding limited across the entire observed area, such that they are $\sim 5.5$ mag deeper in the uncrowded outer regions than in the bulge, in spite of having identical exposure times at all radii.

(A color version of this figure is available in the online journal.)

from neighboring stars as well. In these regimes, the limiting magnitude is no longer set by Poisson counting of electrons in the CCD, but instead is "crowding limited." In crowding-limited data, the depth of the observations increases little with increased exposure time (although the photometry of the detected stars does improve), and only observations with higher angular resolution, or of lower surface density regions can significantly improve the limiting magnitudes. In M31, both the ACS and the WFC3/IR observations will be crowding limited over much of the disk, with the crowding limit being brighter in the higher surface brightness central regions.

We can use the $50 \%$ completeness limits to determine the radial dependence of the survey depth of the survey. We assign a radius to each field by calculating the median right ascension and declination of the detected stars, and then solving for the deprojected major axis length of an ellipse passing through that position (assuming an inclined disk with $i=70^{\circ}$, a position angle of $43^{\circ}$, and a center at $\alpha(2000)=10.68473$ and $\delta(2000)=41.26905$; we discuss the choice of inclination and position angle further in Section 5.2 below). We also calculate the mean number surface density of stars with $\mathrm{S} / \mathrm{N}>4$ (i.e., stars $\operatorname{arcsec}^{-2}$ ) in the st and the culled, high-quality gst catalogs for each field.

We plot the radial dependence of the $50 \%$ completeness magnitude limit in Figure 11. As expected, we see a strong radial dependence in the completeness limit, in spite of all fields having nearly identical exposure times. In both the optical and the NIR, there is a brighter magnitude limit in regions with higher stellar densities. The effective magnitude limit of the ACS filters is more than 4 mag brighter in the bulge than in the diffuse outer disk. The variation in the WFC3/IR channel is even larger, being $~ 5.5$ mag deeper in the outer disk than in the bulge; given the larger pixels of the WFC3/IR camera, it is not surprising that the NIR observations are the most severely affected by crowding.

In contrast, there is little radial variation in the $50 \%$ completeness limit in the WFC3/UVIS data, with only the slightest hint of an upturn in the central bulge field. The limiting magnitude of the WFC3/UVIS data is therefore set by photon counting, not crowding. Longer WFC3/UVIS observations could therefore be expected to yield significantly deeper CMDs, unlike for the PHAT WFC3/IR and ACS/WFC CMDs, which are the deepest that are currently possible at these radii in M31. We note that the observed radial variation agrees extremely well with predictions initially made using Olsen et al.'s (2003) crowding simulations (modified for the PHAT observing parameters) in advance of the start of survey operations.

We can see the impact of stellar density on photometric depth more directly in Figure 12, where we plot the correlation between the $50 \%$ completeness limit and the stellar density of all stars detected with $\mathrm{S} / \mathrm{N}>4$ in a given filter (open circles, from the st catalogs), and for the subset of stars with reliable photometry and $\mathrm{S} / \mathrm{N}>4$ in both of the filters observed with a single camera (solid circles, from the gst catalogs). At high stellar densities, the photometric quality cuts significantly reduce the numbers of stars in the gst catalog compared to the less stringent st catalog, by roughly a factor of three. These cuts have a particularly large impact on the F336W filter because of the requirement that the gst catalog also has $\mathrm{S} / \mathrm{N}>4$ for the shallower F275W observations.

For photon-limited observations, we expect the limiting magnitude in Figure 12 to be constant for a fixed exposure time, and to have no dependence on the stellar surface density. We see this expected behavior in the WFC3/UVIS filters, where there is no correlation between the completeness limit and the stellar density.

The relationship between stellar density and the magnitude limit is far more complicated for the ACS data, which is crowding limited over most of the disk. At the lowest stellar densities shown, there is no obvious relationship between the completeness limit and the stellar surface density, indicating that some of the ACS data have reached a low enough surface density $\left(\lesssim 6\right.$ stars $\left.\operatorname{arcsec}^{-2}\right)$ that the photometry becomes photon limited. These limiting magnitudes are roughly 27.9 in $\mathrm{F} 475 \mathrm{~W}$ and 27.1 in $\mathrm{F} 814 \mathrm{~W}$ for the ACS exposures taken at a single pointing. ${ }^{24}$ At higher stellar surface densities, the limiting magnitude of the ACS data is systematically brighter, as the crowding begins to affect the ability to detect faint stars (the st points), or to produce reliable photometry (the gst points). Instead, the completeness limit becomes pinned to whichever depth produces $\sim 14$ stars $\operatorname{arcsec}^{-2}$ with reliable photometry (i.e., in regions with intrinsically high stellar surface densities, the density of bright stars is sufficiently high that the maximum surface density can be reached with only the brightest stars in the luminosity function, leading to a bright limiting magnitude).

\footnotetext{
24 The overlapping ACS observations from adjacent pointings will increase
} the depths in these photon-limited regions. 

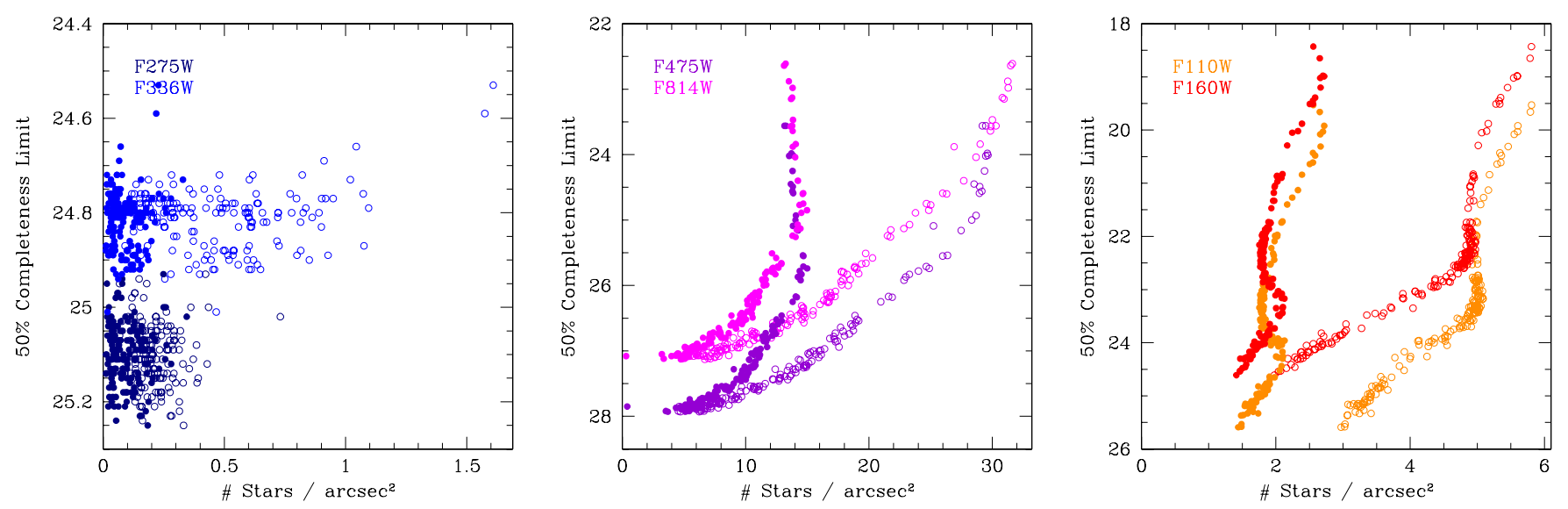

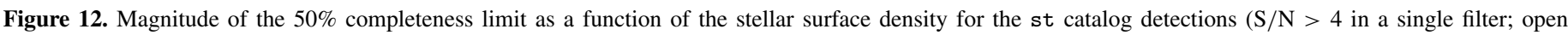

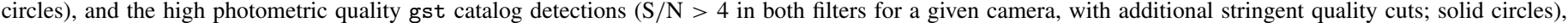

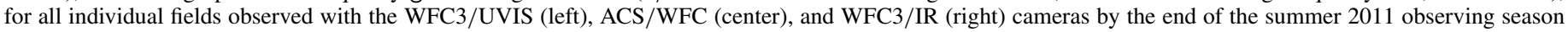

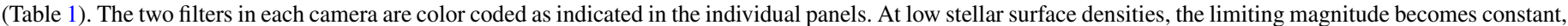

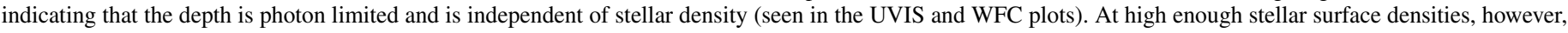

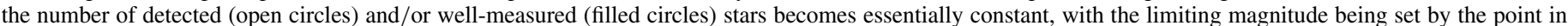

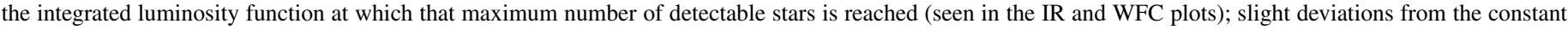

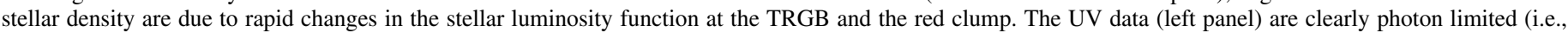

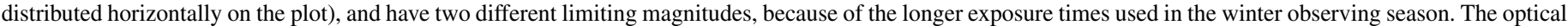

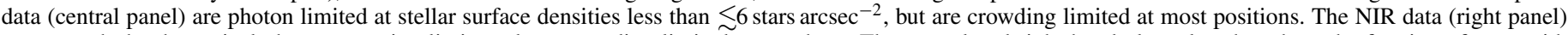

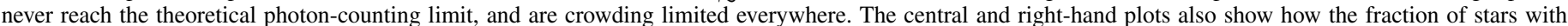
well-measured photometry increases as the stellar density drops and the images become less crowded.

(A color version of this figure is available in the online journal.)

The NIR data in Figure 12 are similar to the optical ACS data in many respects. However, it lacks the turnover at low stellar surface densities to a single $50 \%$ completeness magnitude, indicating that the WFC3/IR data are crowding limited throughout the disk, as was expected from our initial simulations. The surface density of reliably measured stars is essentially constant at $\sim 2$ stars $\operatorname{arcsec}^{-2}$ throughout the galaxy. The small ripples in the stellar surface density at $\sim 24.3 \mathrm{mag}$ in F110W and $\sim 23.2 \mathrm{mag}$ in F160W are due to the increasing numbers of stars at the magnitude of the well-populated red clump, and the bright ripples at 19th and 20th magnitude (in F110W and F160W, respectively) are due the increasing numbers of stars at the NIR TRGB. Hints of a bump caused by the red clump is also visible in the ACS data (at $\sim 24.8$ mag in F814W), but it is less pronounced, because the red clump is somewhat blurred by dust in the optical, making it a less pronounced feature in the luminosity function.

Figure 12 also shows the substantial penalty that results from the larger WFC3/IR pixel scale. At the crowding limit, the ACS camera produces reliable photometry for $\sim 7$ times as many stars $\operatorname{arcsec}^{-2}$ than the WFC3/IR channel. Deeper NIR data in M31 will only be possible with a telescope + camera system with smaller pixels and higher angular resolution.

\subsection{Astrometry}

Producing accurate six-filter photometry for crowded-field photometry requires exquisite sub-pixel alignment between overlapping images. We have made careful astrometric solutions to place our PHAT data on a universal reference frame with an absolute error of 100 mas (dominated by errors in the groundbased reference catalog) and relative errors of 10 mas between stars observed in the three cameras. The astrometric alignment is a multi-stage process in which individual exposures within a field are aligned to each other, overlapping regions within bricks are used to tie the fields together, and finally bricks are aligned to a ground-based absolute astrometric reference frame. We describe each of these steps below.

\subsubsection{Alignment of Individual Exposures taken with One Camera During One Visit}

As discussed briefly above, the individual exposures in an image stack are aligned within the DOLPHOT processing module. DOLPHOT identifies bright stars within each exposure, and then uses the geometric distortion correction to calculate relative shifts to apply to each exposure, based upon initial guesses generated from the astrometry in the header. Typically hundreds to thousands of stars are used in the alignment, with $\sim 300,525,850,800,6000$, and 6250 average alignment stars for the F275W, F336W, F475W, F814W, F110W, and F160W filters, respectively; the number of alignment stars used for the NIR filters varies dramatically with radius from $\sim 2000$ in the outer disk to 20,000 in the inner bulge. The resulting corrections to the (relative) header-based astrometry are typically of the order of 1-2 pixels or less. The only exceptions found in the data so far are for the ACS images in Brick 21, Field 2, which have relative shifts that differ substantially from what would be inferred from the headers; data quality in this field is otherwise unexceptional. For WFC3/UVIS and ACS/WFC, each chip is aligned independently. The rms of the shifts for pairs of alignment stars are typically $\sim 0.2$ pixels for WFC3/UVIS and ACS/WFC, and 0.1 pixels for WFC3/IR.

\subsubsection{Aligning All Images for a Single Camera}

The photometric catalogs initially generated by DOLPHOT contain distortion-corrected astrometry, tied to the astrometry of a reference frame generated with MULTIDRIZZLE. These reference frames, in general, are translated and rotated with respect to a global reference frame (due to errors in guide star catalog positions) and also have shear, scale, and distortion (due to residuals from the pipeline distortion corrections). We aim to remove these errors by aligning the individual catalogs with each other and with an astrometric reference catalog tied to a global reference frame. To align the astrometry we use the gst catalogs for ACS/WFC and WFC3/IR, since they contain fewer 
spurious stars, and the st catalogs for WFC3/UVIS, with cuts on the F336W detections only $(\mathrm{S} / \mathrm{N}>4$ and crowd $\leqslant 0.25)$ to avoid eliminating the large number of valid $\mathrm{F} 336 \mathrm{~W}$-detected stars that were undetected in the shallow F275W imaging. We split the ACS/WFC catalogs along the CCD gap and handle the two CCDs separately.

We begin by coarsely aligning the individual catalogs in celestial coordinates, allowing only translational corrections. For the large ACS/WFC catalogs, we cut to bright $(\mathrm{F} 475 \mathrm{~W}<$ 24) stars for speed. Given a set of spatially overlapping catalogs, we search each pair of catalogs for pairs of stars (one from each catalog) within $2^{\prime \prime}$ of each other, with this limiting separation set by the expected astrometric uncertainty of the cameras relative to the guide stars. This large matching radius is necessary to handle the typical offsets we find in our data. If the affine (rotation, shear, and scale) and distortion corrections are small, then the distribution of positional differences between the pairs of stars will include a peak (due to true matches) and a background (due to false matches). We also observe a ring of lower match density around the peak due to DOLPHOT's closeness-ofsources limit. The position of the peak in ( $\Delta$ R.A., $\Delta$ decl.) space is the mean positional offset between the two catalogs, and the width of the peak is due to measurement errors plus affine and distortion errors. We fit for ( $\Delta$ R.A., $\Delta$ decl.) using a Gaussian peak plus flat background model using the expectation maximization algorithm (Dempster et al. 1977). Once we have measured the offsets between each pair of overlapping catalogs, we solve a least-squares equation to find a consistent set of translational corrections that will simultaneously minimize the distance between matched pairs of stars for the entire set of catalogs.

\subsubsection{Tying Photometric Catalogs to a Global Astrometric Frame}

After applying the initial translational corrections described above, the contiguous catalogs for each camera are roughly aligned internally. Next, we align the cameras with each other and with a global reference frame. This step is required for eventual comparisons with other multi-wavelength data sets.

We have defined a dense network of global astrometric standards using archival $i^{\prime}$ data from MegaCam Canada-France-Hawaii Telescope (CFHT; courtesy of JeanCharles Cuillandre). The CFHT processed images were subjected to iterative PSF photometry. The photometry in the resulting astrometric catalog extends down to $i^{\prime}=21.5$ and contains 1.7 million sources. For comparison, the Massey et al. (2006) Local Group Survey catalog contains one-seventh as many sources over the same region. The positions of the brighter, non-saturated stars were matched to Two Micron All Sky Survey (2MASS; Skrutskie et al. 2006)-which itself is tied to Tycho-2 (Høg et al. 2000) - to produce a well-constrained astrometric solution for the entire region. The dispersion for matches between CFHT sources with $i^{\prime}<18$ and 2MASS is about 0.2 arcsec.

We begin the global alignment by roughly aligning the ACS/WFC catalogs to the CFHT-based reference catalog. We first merge the ACS/WFC catalogs, removing one star from each pair within 30 mas of each other, and cutting to F814W $<21.5$ to match the depth of the reference catalog. Then, as before, we find stars in the ACS/WFC and CFHT catalogs within $1^{\prime \prime}$ of each other, fit a Gaussian peak plus background model, and use the peak weights to fit a least-squares affine correction (translation, rotation, scale, and shear) to the ACS/WFC catalogs. Similarly, we roughly align the WFC3/UVIS and WFC3/IR catalogs to the ACS/WFC catalogs.

Finally, we repeat the alignment process, this time allowing each individual catalog an affine correction to bring it into alignment with its peers and with the reference catalog. We do this, as before, by finding nearby pairs (both between pairs of catalogs, and between the catalogs and the reference), fitting a Gaussian peak plus background model to properly weight the matches, and computing a least-squares set of affine corrections. $^{25}$

We characterize the accuracy of the photometry as the width of the Gaussian peak fit to the distribution of all offsets between pairs of matched stars. The final alignment of the ACS/WFC catalog to the CFHT reference frame has a $1 \sigma$ accuracy of $\sim 45$ mas per coordinate in the outer disk, increasing to $\sim 60$ mas in the bulge. The internal alignments within the WFC3/UVIS, ACS/WFC, and WFC3/IR catalogs have $1 \sigma$ accuracies of $\sim 8.5$ mas, $\sim 6$ mas, and $\sim 6$ mas, respectively. The relative alignments of the WFC3/UVIS and WFC3/IR catalogs to ACS/WFC have $1 \sigma$ accuracies of $\sim 6$ mas and $\sim 3.7$ mas, respectively.

Even with the inclusion of affine corrections, we see clear structured residuals of up to $\sim 5$ mas when matching different cameras to each other. These residuals have a consistent structure from field-to-field within a brick, and are almost certainly caused by errors in the geometric distortion correction. In future releases, we expect to refine the above procedure to include corrections to this geometric distortion.

\subsubsection{Merging Photometric Catalogs for All Cameras}

For some applications it is helpful to have catalogs of matched multi-camera (six-filter) photometry. This step involves merging measurements made with three different cameras, with multiple overlapping pointings, in highly crowded fields. As a result, this step can only be done after all catalogs have been tied together astrometrically to a high degree of accuracy. We generate merged, six-filter catalogs as follows. We first remove duplicates from catalogs of overlapping fields, for each camera. First, we identify and group any ACS/WFC stars that appear within 50 mas of each other in multiple catalogs. We then replace them with a single ACS measurement assigned to the mean of the measured positions and magnitudes in the overlapping catalogs. We do the same for WFC3/UVIS and WFC3/IR, resulting in a single brick-wide catalog for each camera, with duplicates removed. We then generate the multicamera photometric catalog by matching the merged ACS/WFC stars with the merged WFC3/UVIS and WFC3/IR stars, using a matching radius of 50 mas. Stars are not required to be matched in multiple cameras, such that a source with no counterparts within 50 mas will still be included in the merged catalog.

We note that deblending sources near the detection limit in highly crowded fields does not always produce a unique result, leading to slight variations in the positions and assigned magnitudes of deblended stars. As such, source matching becomes less reliable at faint magnitudes. In future releases, we will be performing source identification and photometry simultaneously across all overlapping images in all three cameras, which should eliminate this problem.

\footnotetext{
25 We note that we have more than ample signal to perform these fits, in spite of the large number of parameters needed for the field-by-field affine transformations. The least-squares problem when aligning a set of 18 ACS/WFC catalogs to the CFHT-based reference typically has $\sim 10^{7}$ matches, but only 216 parameters ( 6 per catalog).
} 

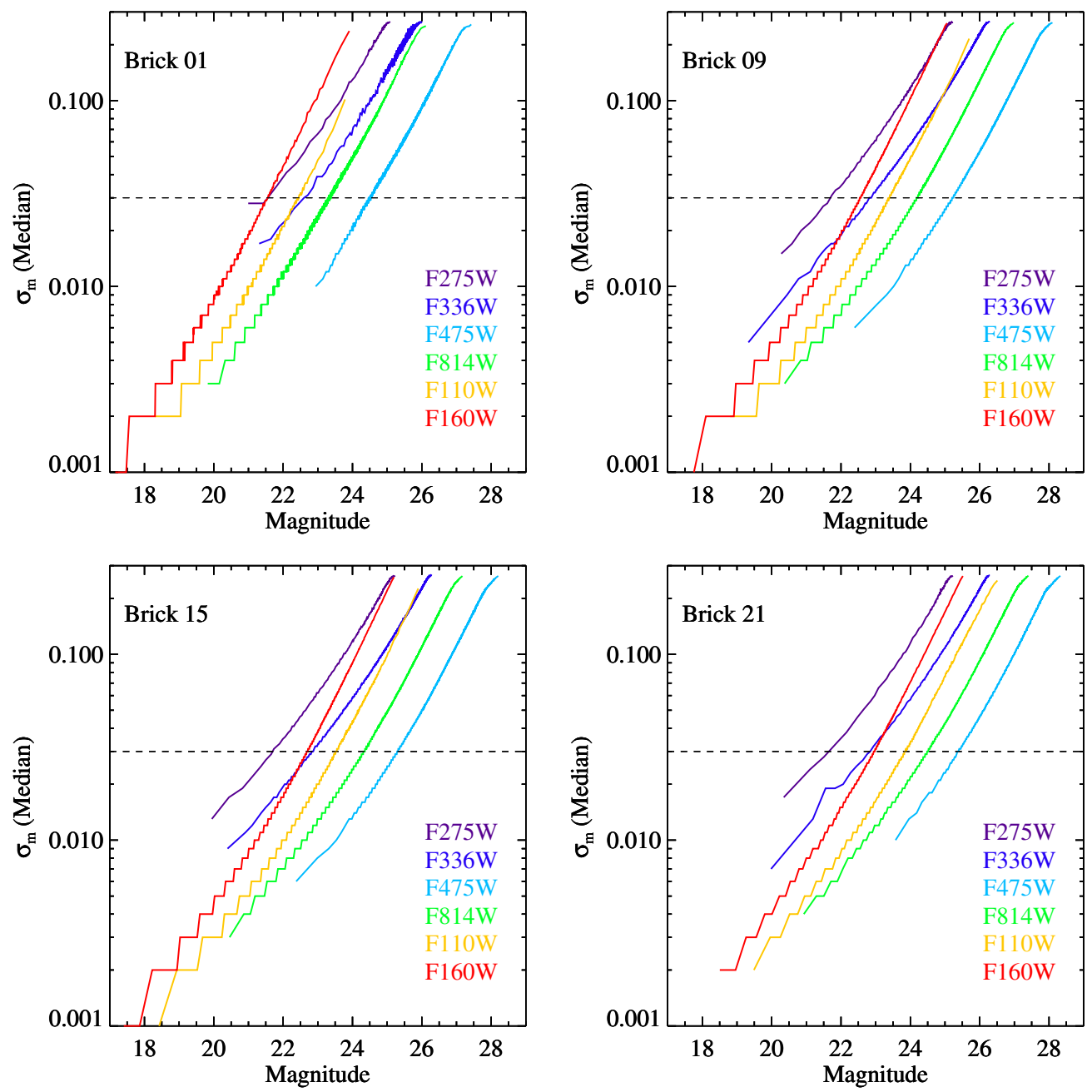

Figure 13. Median photometric errors reported by DOLPHOT in the six PHAT filters, for Bricks 1, 9, 15, and 21 (upper left to lower right). Medians are calculated for groups of 25, 400, or 200 stars, for the UV, optical, and NIR channels, respectively. Photometry is for stars with S/N $>4$ in a given filter, restricted to stars with well-measured photometry (FLAG $=0$ ) that do not fall near the chip gap or the edges of the image. Note that the formal photometric uncertainties underestimate the true uncertainty, which is frequently dominated by crowding errors at the faint end (Figures 14-16), and by systematic errors in the bright end; the horizontal dashed line indicates a characteristic amplitude of systematic uncertainties (Figure 18). The steps at bright magnitudes are due to the limited number of significant figures used for the DOLPHOT photometric uncertainties.

(A color version of this figure is available in the online journal.)

\subsubsection{Propagating Astrometry Back into the Photometric Catalogs}

The result of our astrometric alignment process is an affine transformation for each camera in each field. We apply these affine transformations to the FITS World Coordinate System (WCS) headers of the input *.flt images, along with the small relative shifts that DOLPHOT calculated during alignment of the image stacks. We modify the WCS CRVAL headers to apply the shifts, and the CD matrix elements to apply the remaining affine terms. We also propagate the revised astrometry back to the individual photometric catalogs.

\subsection{Data Quality}

We have taken a number of different steps to characterize the current quality of the photometry. In the following sections we discuss the raw photometric uncertainties reported by DOLPHOT (Section 4.7.1), the uncertainties and biases due to crowding, as assessed by artificial star tests (Section 4.7.2), and uncertainties due to systematic errors, as measured from repeatability tests in overlapping images (Section 4.7.3). All results in this section are for individual fields in the current data release, which do not take advantage of additional depth due to overlapping adjacent fields, or upcoming improvements in the flat fields, distortion corrections, or PSF models. We therefore expect that some of these uncertainties will be reduced in upcoming versions of the PHAT photometry.

\subsubsection{DOLPHOT-reported Uncertainties}

DOLPHOT reports photometric uncertainties for each star as part of its standard output. These uncertainties are based on the Poisson variation in the flux from the star, from the sky, and from neighboring stars whose flux has been subtracted. The value of the uncertainty (in magnitudes) can be found in the MAG1_ERR and MAG2_ERR entries of the gst and st photometric catalogs.

In Figure 13 we plot the median photometric uncertainty as a function of magnitude, for all six filters, in Field 9 of Bricks 1, 9, 15 , and 21 (upper left to lower right). The median uncertainty is given in magnitudes, and was calculated for stars with $\mathrm{S} / \mathrm{N}>4$ in the named filter, using data from the st catalogs. The median uncertainty and magnitude was calculated for groups of 25, 400, and 200 stars, for the WFC3/UVIS, ACS/WFC, and WFC3/IR cameras, respectively. We have not included stars that only appear in a subset of the images (i.e., those that are 
near the edges of an image or near a chip gap); such stars have much higher reported photometric uncertainties, as expected, but are not representative of the bulk of the photometric catalog. We have also excluded stars that touch the edge of an image, or that have saturated pixels (i.e., FLAG $>0$ ).

The median photometric uncertainty in Figure 13 correlates strongly with magnitude, as expected. However, the slope of the correlation is different for each camera, due largely to the changing importance of the sky brightness. In the UV, the uncertainty is dominated by the Poisson variation in the flux from the stars themselves, since the PHAT WFC3/UVIS images are essentially uncrowded and the background of unresolved flux is quite dark. In the optical and NIR, the situation is quite different, due to a high level of crowding and brighter backgrounds. The correlation between photometric uncertainty and magnitude therefore follows a different slope than in the UV. Moreover, the exact relation changes with radius as well, due to the darker background and lower stellar density at large radii.

We note that the photometric uncertainties shown in Figure 13 are not always the dominant source of error. For faint stars, flux from unresolved stars below the detection limit can significantly bias the resulting magnitudes. For bright stars, systematic uncertainties in the flat field, CTE corrections, or PSF model can dominate over the formally small photometric error. As we show below in Section 4.7.3, these systematic errors have typical amplitudes of 0.02-0.04 mag for bright stars; we include a horizontal line in Figure 13 to indicate the approximate magnitude range where systematic uncertainties will become important.

\subsubsection{Crowding Uncertainties and Biases from Artificial Stars}

The accuracy of stellar photometry can be strongly affected by crowding in regions of high stellar density. In such cases stars that are too faint to be detected individually can blend with other stars to rise above the detection limit. Thus, many of the faint stars in the photometric catalog have been biased to brighter magnitudes by their neighbors. High crowding also makes it difficult to accurately measure the local background, because all of the pixels outside a star's photometric aperture are filled with other stars. Finally, crowding tends to increase the photometric uncertainty, because the measured flux depends on the accuracy with which the flux from all surrounding stars can be subtracted from the image.

The only way to accurately assess the effect of crowding is through extensive artificial star tests (described above in Section 4.5). These tests allow us to measure the difference between the true and recovered magnitude as a function of the star's color, magnitude, and position.

In Figures 14-16, we show a series of three-panel plots containing the cumulative distributions of the magnitude difference (left panels), the fractional flux difference scaled by the flux uncertainty (center panels), and the color difference (right panels) as a function of magnitude (where redder colors and thicker lines indicate fainter stars), for the UV (Figure 14), optical (Figure 15), and NIR (Figure 16), using Field 9 of Bricks 1, 9, 15 , and 21 (top to bottom, respectively). We only include data for Brick 1 in the UV analysis; the WFC3/UVIS data are uncrowded at all radii outside the very central regions of the bulge, and thus the artificial star tests yield identical results at all radii. All quantities are given as the measured value minus the true value for the inserted star. Photometry only includes stars from the gst catalogs.
Figure 14 shows that the effect of crowding is minimal for the F275W and F336W filters. The differences between the input and output magnitudes and/or colors increase for fainter stars (left and right panels, respectively). However, if one scales the flux difference by the photometric uncertainty reported by DOLPHOT (center panel), it is clear that the distribution of flux differences is essentially Gaussian, with a width set by the photon-counting uncertainties alone. The only sign of crowding effects is an extremely slight tail toward brighter fluxes at faint magnitudes in the deeper F336W filter (thick red line in the central panel of the bottom row).

The effects of crowding are more severe in the optical, as can been seen in Figure 15. Unlike in the UV, the distributions of flux differences (center panels) do not follow the Gaussian distribution expected for uncertainties dominated by photon counting (with the possible exception of the brightest stars in the least crowded fields). The distributions are instead skewed, such that stars are preferentially detected as brighter than their true magnitude. The skewing yields a significantly increased probability that a star will be reported as being brighter than expected for photon-counting uncertainties (i.e., compare the fraction of stars that are more than $5 \sigma$ brighter to either the Gaussian expectation or to the fraction that are $5 \sigma$ fainter). However, the median magnitude difference is not highly biased outside of the bulge, and the median flux errors are typically biased by less than the expected photometric error. The effects of crowding are noticeably worse in the inner regions of the galaxy, where the surface density is highest. Crowding also has more of an effect in the F814W filter, which has a larger PSF, and which is deeper than F475W, and thus has a higher density of resolved stars.

The crowding errors are largest in the NIR (Figure 16), which suffers from worse resolution than either ACS or WFC3/UVIS, due to bigger pixels and an intrinsically broader PSF at long wavelengths. As a result, the effects of crowding are severe at all radii, even the outer disk. These effects can be clearly seen in the distributions of magnitude errors, which are dramatically wider than expected from photon counting along (middle panels), and which show clear skewing (left panels).

Surprisingly, the NIR photometry is biased toward fainter magnitudes for the median star. The bias is slight in F110W, but noticeable in F160W, where the crowding is most severe. This difference between the two filters then produces a slight blueward shift in the median F110W-F160W color $(<0.05$ mag in all but the faintest bin). In all cases, the systematic bias in the median magnitude or color is significantly smaller than the width of the error distribution, such that the error properties will be dominated by random rather than systematic errors in most applications. The systematic bias is also of the same order as both the intrinsic errors in repeat measurements (see Section 4.7 .3 below) and the likely uncertainties in the current zero points. As such, while the biases in Figure 16 are undesirable, they are unlikely to be the dominant source of systematic errors, except perhaps in the densest regions in the bulge. Unfortunately, we do not currently have a convincing explanation for this offset at this point. We are continuing to investigate and expect to have the issue resolved for the second data release.

\subsubsection{Repeatability and Systematic Uncertainties}

There are a number of position-dependent uncertainties that are not reflected in the DOLPHOT-reported uncertainties or the artificial star tests, but that will contribute to magnitude 

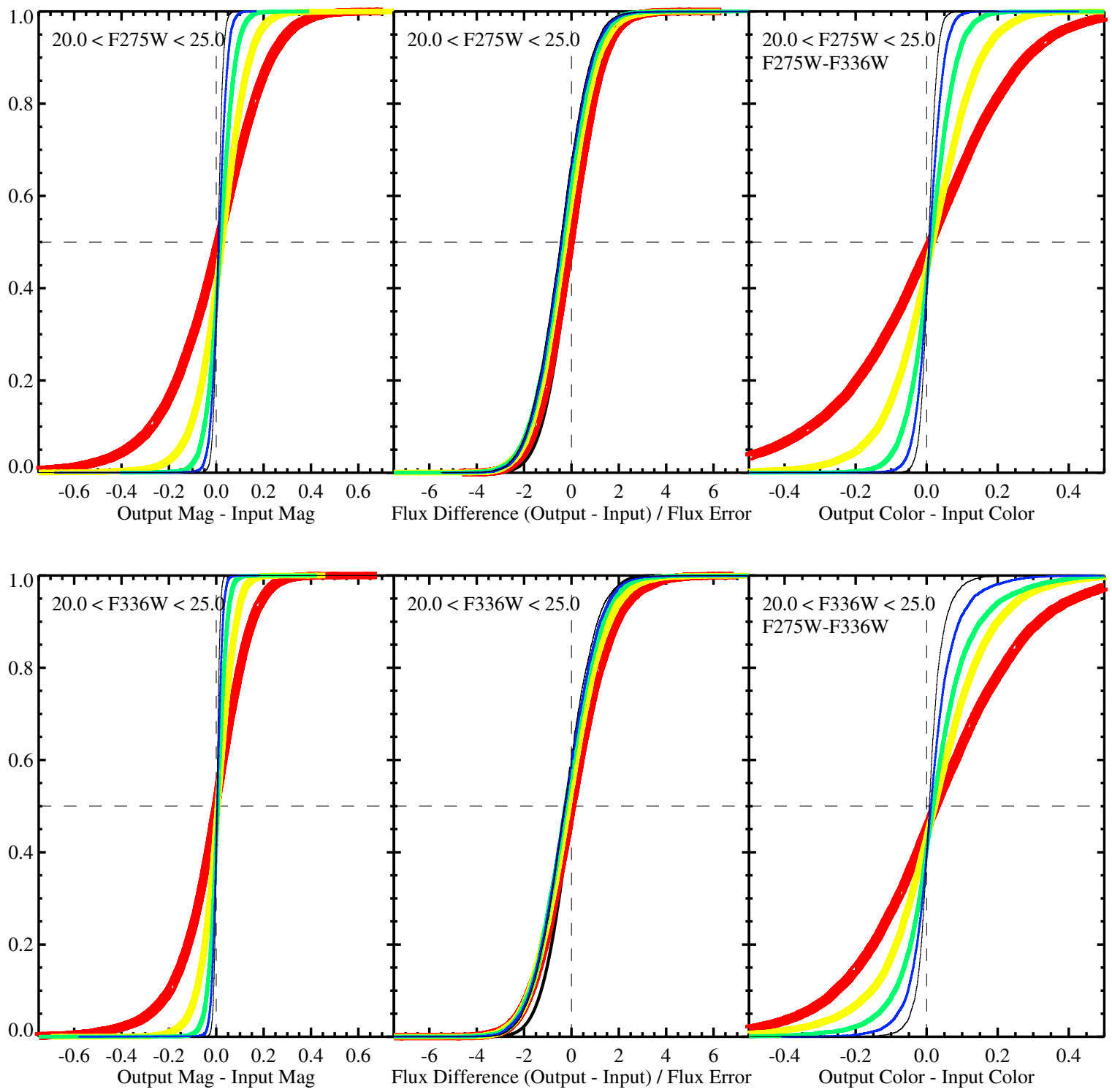

Figure 14. Comparisons between the input and output properties of artificial stars inserted into WFC3/UVIS images in Brick 1, Field 9, for F275W (top row) and F336W (bottom row). Panels show the cumulative distribution of (left) the difference between the recovered and inserted magnitude, such that negative numbers indicate stars that are recovered brighter than their true magnitude; (center) the fractional difference in flux, scaled by the photometric uncertainty reported by DOLPHOT such that positive numbers indicate stars that are recovered brighter than their true magnitude; and (right) the difference between the recovered and the input color of the artificial stars, such that positive values indicate stars that are recovered redder than their true color. Each curve shows the distribution for artificial stars with recovered magnitudes in a $1 \mathrm{mag}$ wide range, spanning the total magnitude interval specified on each panel, color coded such that fainter stars are plotted with redder colors and thicker lines (i.e., the top row analyzes bins starting with stars having $20<m_{\mathrm{F} 275 \mathrm{~W} \text {, out }}<21$ (thin purple line), down to $24<m_{\mathrm{F} 275 \mathrm{~W} \text {,out }}<25$ (thick red line)). The center panel also includes a black line showing the expectation for a Gaussian with a width set equal to the median photometric error of stars in the bin. The UV data follow the Gaussian expectation well, because of the lack of crowding in the UV data everywhere outside the very center of the bulge.

(A color version of this figure is available in the online journal.)

differences when the same star is measured in different parts of a single chip. These include errors in the flat field, the positiondependent TinyTim model for the PSF, the CTE correction, and the image-to-image DOLPHOT aperture corrections. These will manifest in the distribution of magnitude differences as a constant systematic error that adds linearly with the random photometric error. We can test for these effects via the repeatability of our photometry when comparing the magnitudes of individual stars in overlapping observations.

We use the PHAT astrometry to identify all pairs of matched stars from the gst catalogs whose positional offsets give them a better than $75 \%$ chance of being a true match, based on the observed distribution of astrometric offsets. These matched pairs sample the entire area of the ACS/WFC due to the large amount of overlap between adjacent fields. In the WFC3/UVIS camera, the matched pairs cover more than $50 \%$ of the chips, but do not sample a square "hole" in the center of the FOV. In the WFC3/IR channel, the matched pairs only sample the very edges of the FOV $\left(\sim 3^{\prime \prime}-5^{\prime \prime}\right)$ due to their limited overlap (see Figure 5). Thus, one should be aware that only the results for ACS/WFC can be seen as truly representative of the entire FOV, whereas for the WFC3/IR channel, we are only probing the extreme edges of the chip, where many of the systematic effects are likely to be at their worst.

In Figure 17 we plot the cumulative distribution of observed magnitude differences between repeated observations of stars within limited magnitude ranges (i.e., bins of $0.5 \mathrm{mag}$, with bluer lines indicating brighter magnitude ranges). We also 

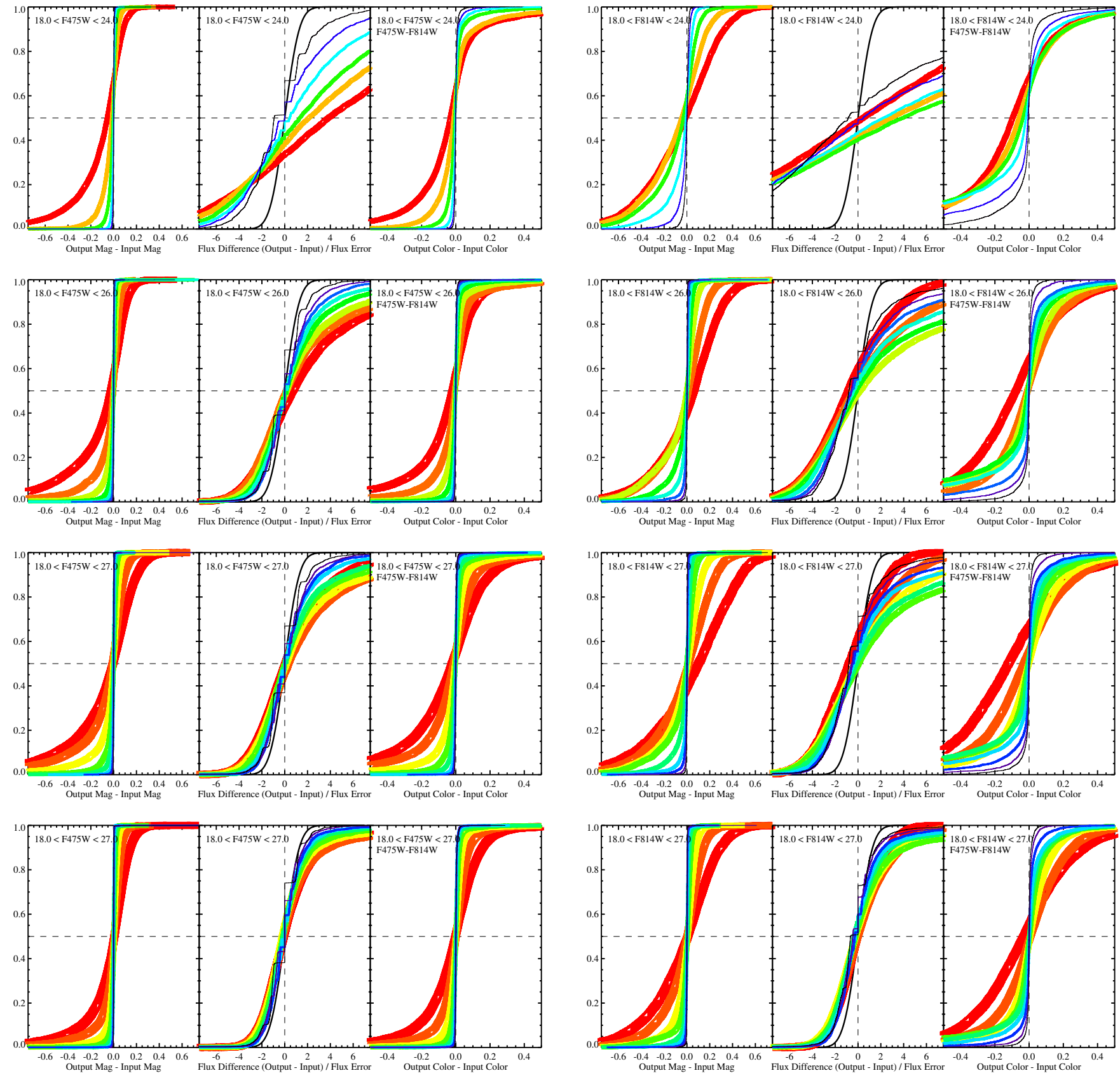

Figure 15. Comparisons between the input and output properties of artificial stars inserted into ACS/WFC images in Field 9 of Bricks 1,9, 15, and 23 (top to bottom rows, respectively), for F475W (left column) and F814W (right column). Panels show the cumulative distribution of (left) the difference between the recovered and inserted magnitude, such that negative numbers indicate stars that are recovered brighter than their true magnitude; (center) the fractional difference in flux, scaled by the photometric uncertainty reported by DOLPHOT such that positive numbers indicate stars that are recovered brighter than their true magnitude; and (right) the difference between the recovered and the input color of the artificial stars, such that positive values indicate stars that are recovered redder than their true color. Line types indicate different bins in recovered magnitude, as described in Figure 14. Unlike the UV data, the optical data depart dramatically from a Gaussian due to the effects of crowding, in all but the outermost regions (see the middle panel of each three-panel plot). There is a tail of stars recovered with a flux that is significantly larger than their true flux, due to contributions from nearby stars. This bias can be several times larger than the DOLPHOT photometric error for faint stars in highly crowded regions.

(A color version of this figure is available in the online journal.)

plot the $1 \sigma$ widths of the distributions as a function of the median magnitude in each bin (black line), derived from the observed interquartile range of the observed distribution, assuming a Gaussian distribution. These widths are compared with the expected width of the distribution, based solely on photon-counting statistics (blue line). The quadrature difference between the observed and expected width (red line) is then a measure of the systematic error (although an imperfect one, since systematic errors are unlikely to be strictly Gaussian).
These measurements are shown for Brick 15, but the results are representative of what we see in other disk fields with very different crowding levels.

Figure 17 shows several clear trends. First, the distribution of magnitude differences becomes systematically wider at faint magnitudes. However, comparing the observed to the expected width (black versus blue line) shows that the majority of this trend is driven by the larger variation in photon counting for small fluxes. Second, at bright magnitudes, the observed 

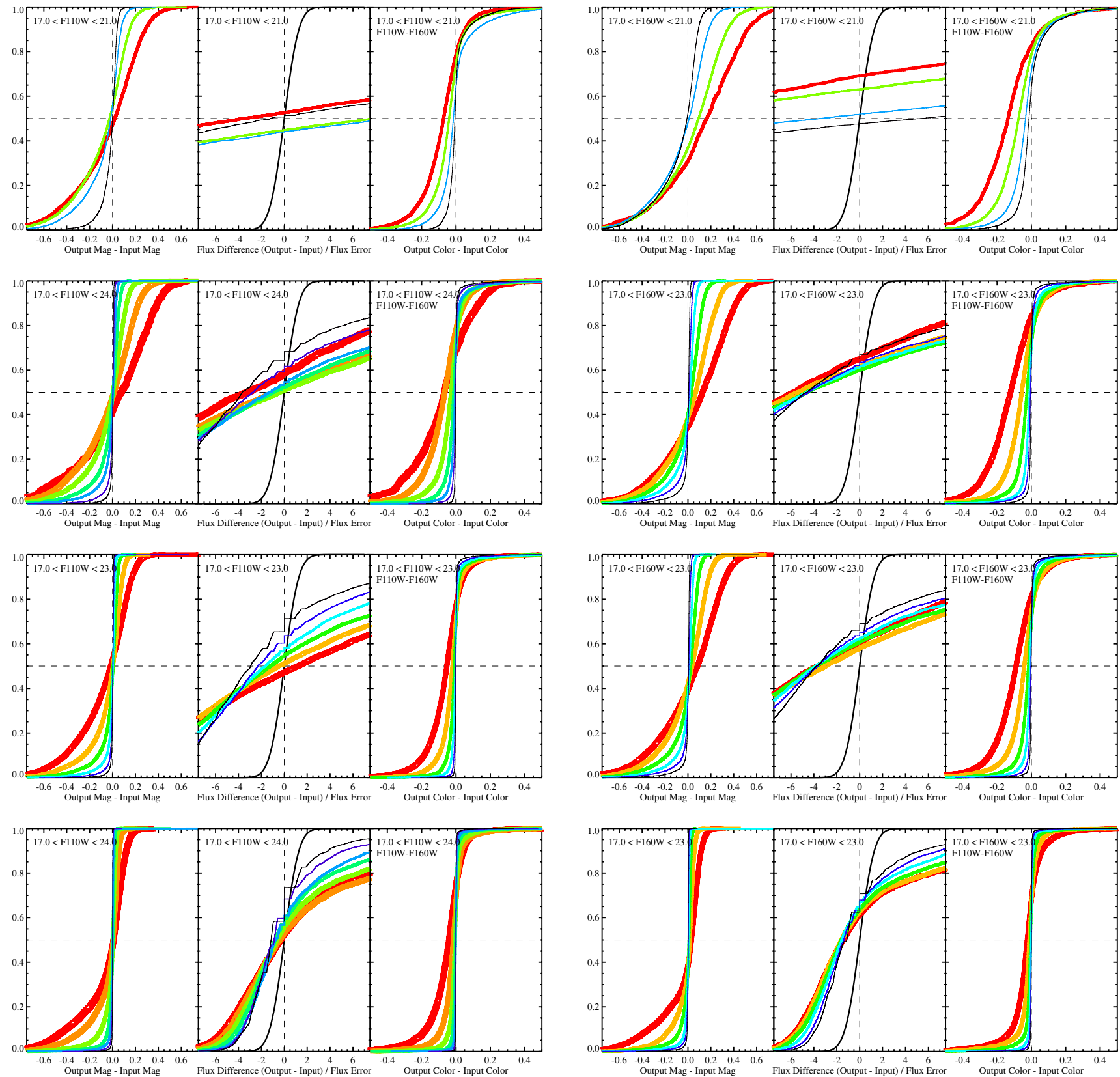

Figure 16. Comparisons between the input and output properties of artificial stars inserted into WFC3/IR images in Field 9 of Bricks 1, 9 , 15, and 23 (top to bottom rows, respectively), for F110W (left column) and F160W (right column). Panels show the cumulative distribution of (left) the difference between the recovered and inserted magnitude, such that negative numbers indicate stars that are recovered brighter than their true magnitude; (center) the fractional difference in flux, scaled by the photometric uncertainty reported by DOLPHOT such that positive numbers indicate stars that are recovered brighter than their true magnitude; and (right) the difference between the recovered and the input color of the artificial stars, such that positive values indicate stars that are recovered redder than their true color. Line types indicate different bins in recovered magnitude, as described in Figure 14. The WFC3/IR data depart dramatically from a Gaussian due to the effects of crowding, everywhere in the disk (see the middle panel of each three-panel plot). The effect is much worse than in the ACS data, due to the larger WFC3/IR pixel size. Moreover, the median star is recovered with a flux that is significantly fainter than its true flux, although crowding does induce the expected tail to brighter fluxes.

(A color version of this figure is available in the online journal.)

magnitude variations are much larger than expected from the formal photometric errors, by factors of 10 in some cases. This difference is a clear signal of systematic errors (red line). However, the systematic errors are small $(\lesssim 0.05 \mathrm{mag})$ at bright magnitudes, where it is easiest to assess their amplitudes. Finally, photometric errors begin to dominate at faint magnitudes in most cases. The magnitude where photometric magnitudes begin to dominate depends on the camera, filter, and degree of crowding. For example, the WFC3/IR observations are crowding limited, and thus can only detect stars much brighter than the true photometric limit of the observations (see Section 4.5.2).
Thus, the WFC3/IR detected stars tend to have lower average photometric uncertainties, making the systematic uncertainties proportionally more important.

We have calculated the width of the observed distribution of color differences in a similar fashion. Because spatial-dependent biases in flat fields and PSF models are often correlated between filters, systematic biases in colors may in fact be smaller than those for magnitudes alone. ${ }^{26}$ In Figure 18 we compare the inferred level of systematic bias in color (black line) to those

\footnotetext{
${ }^{26}$ We see this effect for crowding errors as well (Section 4.7.2).
} 

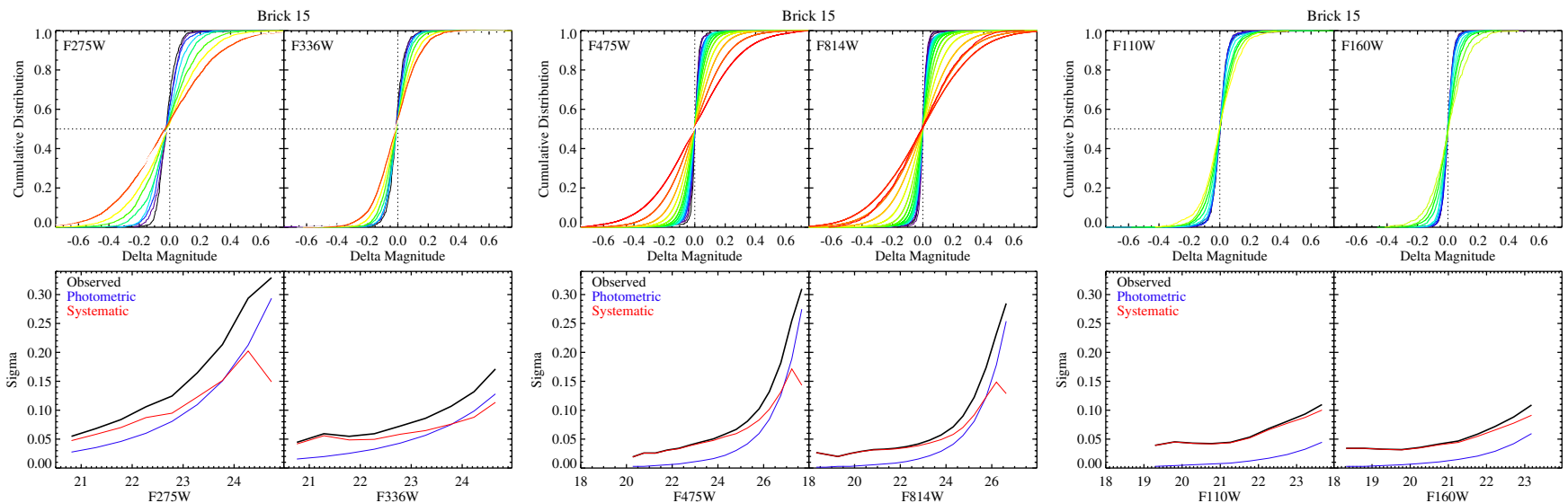

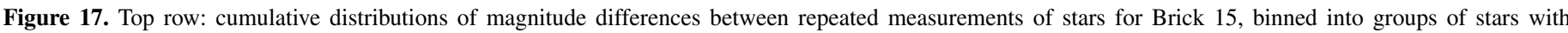

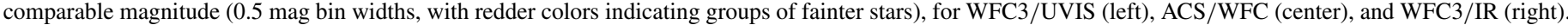

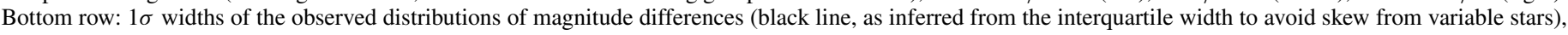

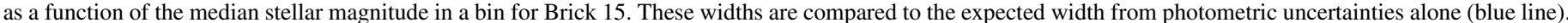

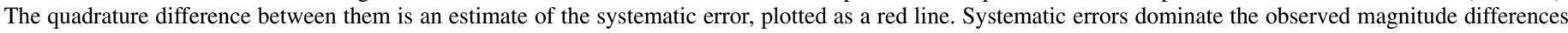

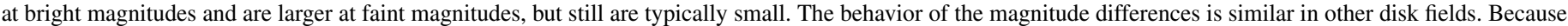

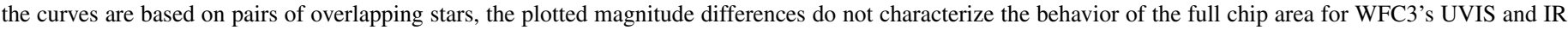
channels, which have limited spatial overlap.

(A color version of this figure is available in the online journal.)

Brick 15

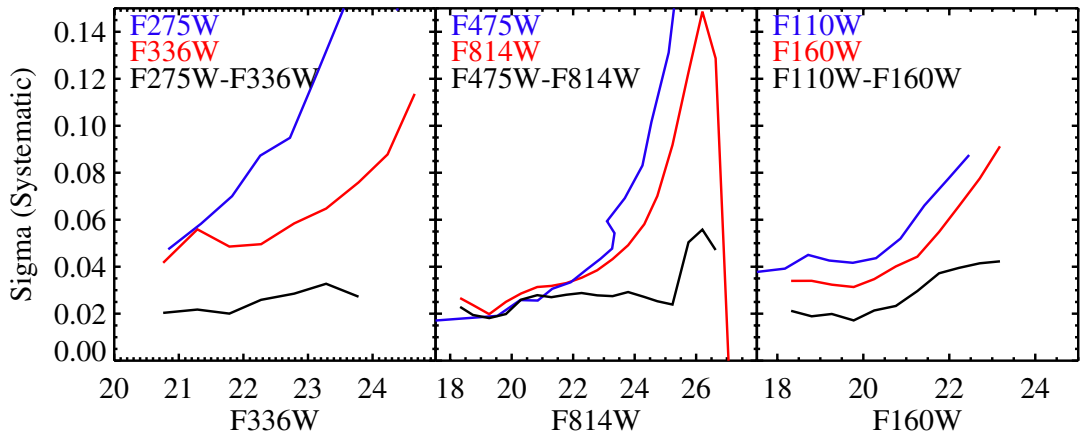

Figure 18. Inferred systematic errors as a function of magnitude for WFC3/UVIS (left), ACS/WFC (center), and WFC3/IR (right), measured in Brick 15. The blue and red lines indicate the systematic error in magnitude measurements for the bluer and redder filter in a single camera, respectively (see Figure 17). The black line shows the systematic error in the color. The systematic uncertainties are typically smaller in color than in magnitude, because many of the positionally dependent uncertainties (flat fields, PSF models) are likely to correlate between the two filters, and thus partially cancel out. The behavior of the magnitude differences is similar in other disk fields. Because the curves are based on pairs of overlapping stars, the plotted magnitude differences do not characterize the behavior of the full chip area for WFC3's UVIS and IR channels, which have limited spatial overlap (see Figure 5).

(A color version of this figure is available in the online journal.)

in the magnitudes of individual filters (blue and red lines, for the bluer and redder filters used for an individual camera). The systematic errors in colors are indeed smaller than those for individual magnitudes, by up to a factor of two in the NIR. They have typical amplitudes of only $0.02 \mathrm{mag}$, rising to $\sim 0.04 \mathrm{mag}$ in the faintest bins. They also appear to have a weaker dependence on the brightness of a star, and are thus more constant with magnitude.

We can use stars that are measured in multiple images to assess whether the systematic biases in Figure 18 have spatial structure, as one would expect for flat-fielding errors or spatially dependent errors in the PSF model. For ACS, we have the benefit of highly overlapping images, such that an individual star appears in multiple quadrants of the ACS chips, and such that every position on each chip has overlapping data. We use these overlaps to assess whether there are locations in the ACS FOV where stars are consistently measured to be brighter or fainter. We first measure the magnitude difference $\delta m$ for each pair of overlapping images for each star in the matched astrometric catalogs. If we assume that this magnitude difference results entirely from systematic errors, then we can map out, in pixel coordinates, the magnitude corrections required to minimize all the $\delta m$ residuals. We do this by spatially binning the $\delta m$ values based on the pixel positions of the two measurements of each star. Weighting using the geometric mean of the errors in the two magnitude measurements, and rejecting $>3 \sigma$ outliers, we find the maximum-likelihood magnitude corrections on a $100 \times 100$ grid in pixel space.

The resulting maps for Bricks 15 and 21 are shown in Figure 19 (top and bottom row, respectively) for F475W (left) and F814W (right). If there were no spatially varying systematics, the $\delta m$ values in each bin would be randomly distributed around zero and our correction map would have small random values. Instead, we find coherent spatial patterns, at the $\sim 0.03$ mag level, that are consistent between bricks. The amplitude of the residual structure is clearly worse for F475W than for F814W. We believe that this difference most likely reflects the better large-scale flat fields that are available for 

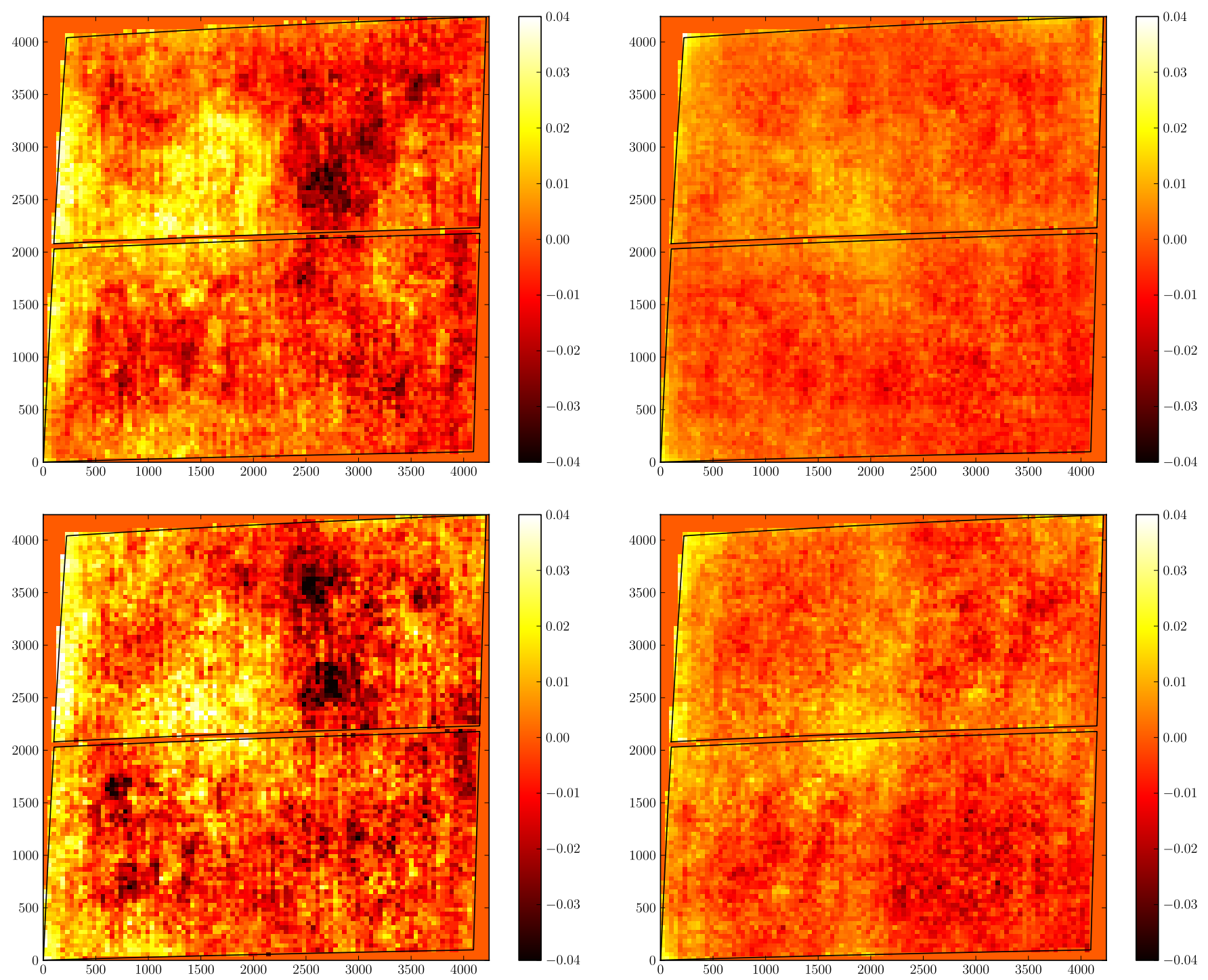

Figure 19. Map of magnitude corrections as a function of position on the ACS reference image for F475W (left column) and F814W (right column), and Bricks 15 and 21 (top and bottom rows, respectively). The color bar on the right refers to the correction, in magnitudes, required to minimize the differences between our measurements for stars that appear in multiple images. The map of F814W offsets is quite smooth. In contrast, the F475W filter shows strong positionally dependent variations that are consistent between the two bricks. These are likely to result from the lack of a large-scale flat-field correction for the F475W filter in the standard STScI flat field.

(A color version of this figure is available in the online journal.)

F814W, which was calibrated against ground-based globular cluster observations (ISR ACS 2002-008 by J. Mack et al.; ISR ACS 2006-06 by R. Gilliland et al.). Even in F814W, however, there are small $\lesssim 0.01 \mathrm{mag}$ systematics associated with the chip gap; hints of these also have been reported in the analysis in ISR ACS 2003-10 by R. van der Marel (see Figure 2). The maps in Figure 19 also indicate larger systematic errors where the boundaries of individual images lie in the distortion-corrected multi-drizzled reference images; stars that fall on these image edges are more likely to have unreliable photometry, and thus it is not clear whether the offsets at those positions reflects flatfielding errors, or the difficulty in doing reliable photometry near the edge of a chip. Even the largest offsets, however, are still less than 5\%.

In future releases, we expect to incorporate new large-scale flats based upon maps like those in Figure 19. Unfortunately, we cannot generate equivalent maps for WFC3, due to the lack of overlapping data that covers the whole FOV for each chip.

\section{RESULTS}

\subsection{Color-Magnitude Diagrams}

The stellar populations revealed by the PHAT imaging are best understood through CMDs produced from the photometric catalogs. In this section we present CMDs for a range of radii within M31. To facilitate interpretation of these data in the following discussion, we include in Figure 20 artificial CMDs generated for the PHAT filter set, showing the positions of stars as a function of their age and metallicity (for $A_{V}=0$ and a constant SFR, assuming photometric errors and limits based on artificial star tests from Brick 23). We also show the expected Galactic foreground populations in Figure 21, calculated with TRILEGAL's default MW model (Girardi et al. 2005; assuming no photometric errors, but applying depth cuts to approximate those in Figure 20).

In what follows, we show CMDs for stars in Bricks 1, 9, 15, and 21, drawn from the gst catalogs. For reference, Bricks 9, 

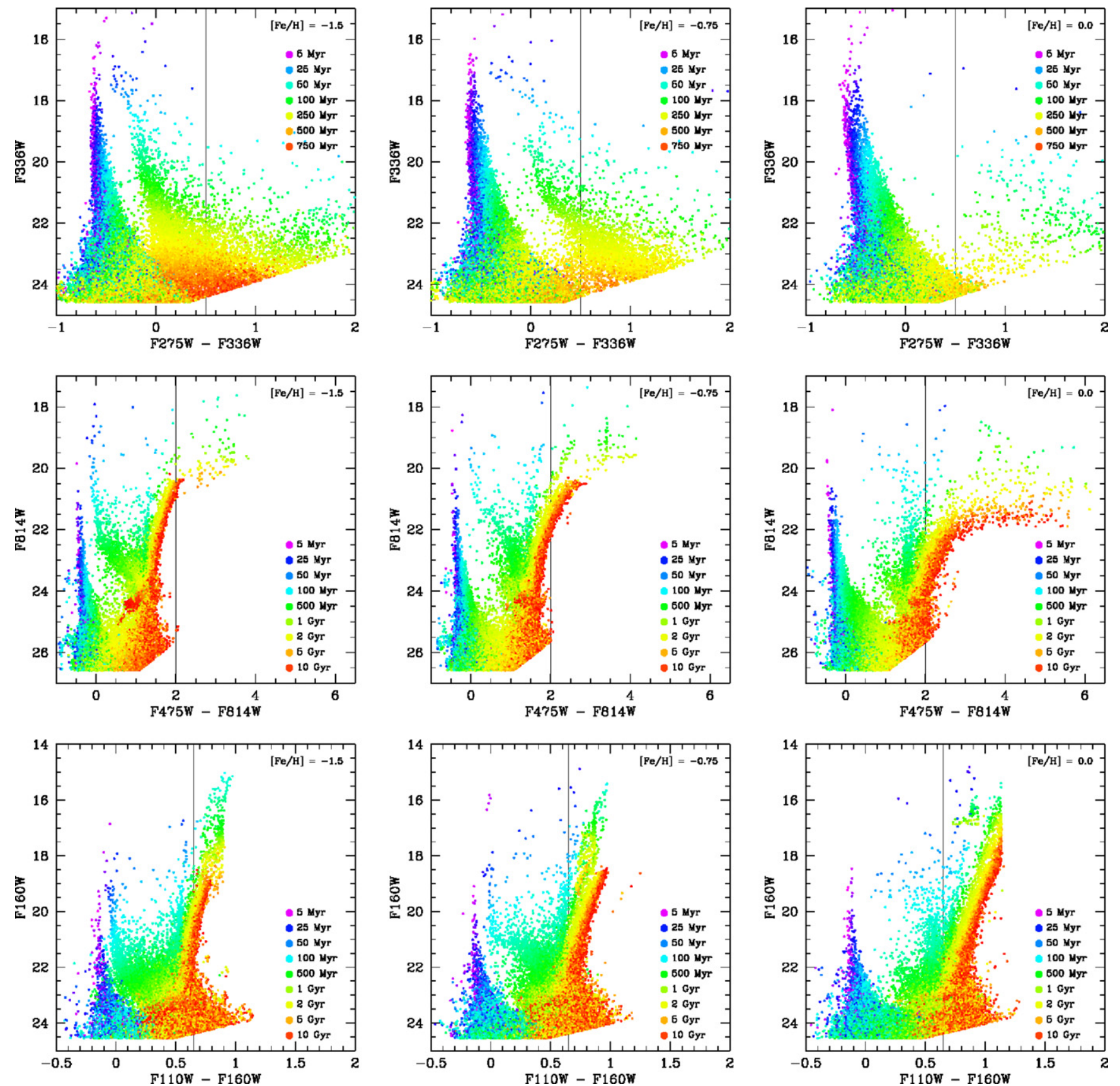

Figure 20. Simulated CMDs for WFC3/UVIS (top row), ACS/WFC (middle row), and WFC3/IR (bottom row), for three different metallicities $([\mathrm{Fe} / \mathrm{H}]=-1.5$, -0.75 , and 0.0 ; left, middle, and right columns, respectively), with points color coded by age. The simulations assume a constant star formation rate, no foreground or internal extinction, and photometric errors and completeness derived from artificial star tests for outer disk fields in Brick 23 . The solar-metallicity CMDs are most likely to be characteristic of the young stellar populations throughout the disk, and the older stellar populations in the bulge. The intermediate metallicity $([\mathrm{Fe} / \mathrm{H}]=-0.75) \mathrm{CMDs}$ are likely to be representative of the older thin and thick disk stars (e.g., Collins et al. 2011), and the low-metallicity CMDs are likely to be characteristic of a potential stellar halo population (Chapman et al. 2006; Kalirai et al. 2006a). Age color coding is the same for all plots in a single row, but varies from camera to camera. Vertical lines are plotted at a constant color for each camera, and are shown for reference, to allow comparisons between different metallicities and with foreground populations shown in Figure 21.

(A color version of this figure is available in the online journal.)

15, and 21 are sampling a series of M31's star-forming rings at progressively larger radii, whereas Brick 1 is almost entirely dominated by the bulge (Figure 1). The stellar surface density varies dramatically among these regions, with higher numbered bricks having systematically lower stellar surface densities, and thus deeper CMDs in the optical and NIR, due to reduced crowding (Figure 11). There are also significant variations in the stellar density within Brick 1 itself, leading to highly nonuniform limiting magnitudes within this single brick.

\subsubsection{Brick-wide CMDs}

In Figures 22-25, we show Hess diagrams for the UV, optical, and NIR, for stars in Bricks 1, 9, 15, and 21 (top left to bottom right).

The UV disk CMDs (Figure 22) are dominated by a clear diagonal sequence of main-sequence stars, extending down to $\mathrm{F} 336 \mathrm{~W} \sim 24.5$ (corresponding to a $\sim 3 M_{\odot}$ star at the distance of M31). These young stars have ages of $\lesssim 500 \mathrm{Myr}$ 

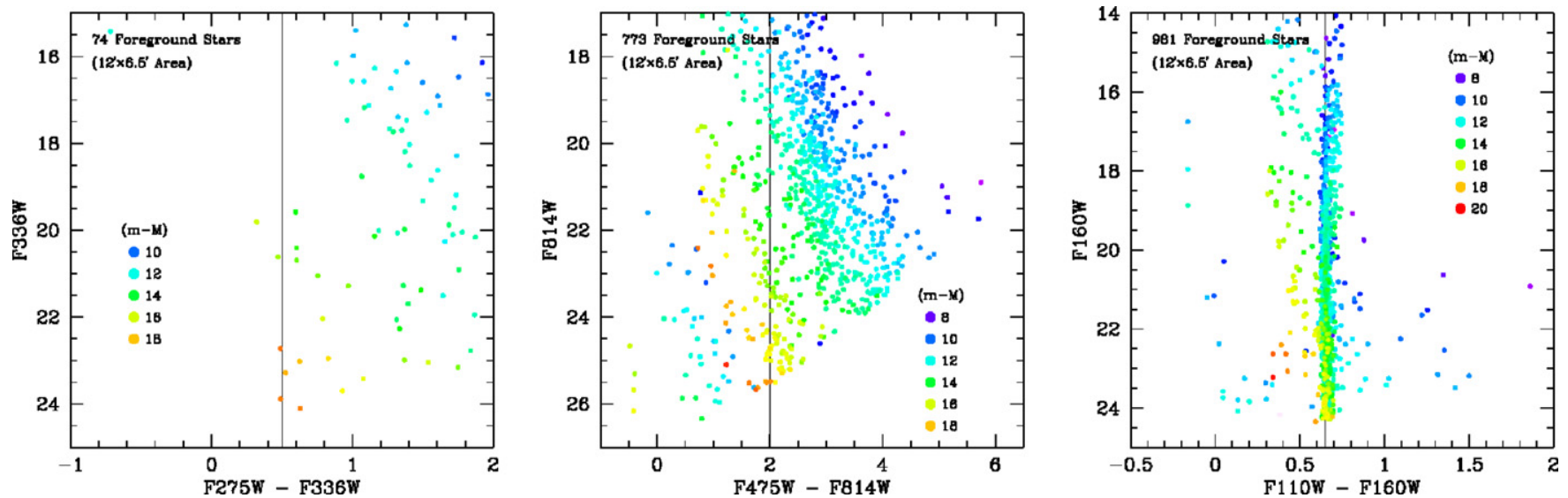

Figure 21. Simulated CMDs for expected foreground populations in WFC3/UVIS (left), ACS/WFC (center), and WFC3/IR (right), calculated from TRILEGAL's default Milky Way model (Girardi et al. 2005). CMDs assume a default area of $12^{\prime} \times 6.5$, corresponding to the $3 \times 6$ WFC $3 /$ IR footprint of a single brick; the number of stars in each CMD is indicated in the plots. Stars in each panel are color coded by their distance modulus $(m-M)$. The color and magnitude ranges are identical to those in Figure 20. Vertical lines are shown for reference to allow comparisons with simulated stellar populations shown in Figure 20.

(A color version of this figure is available in the online journal.)
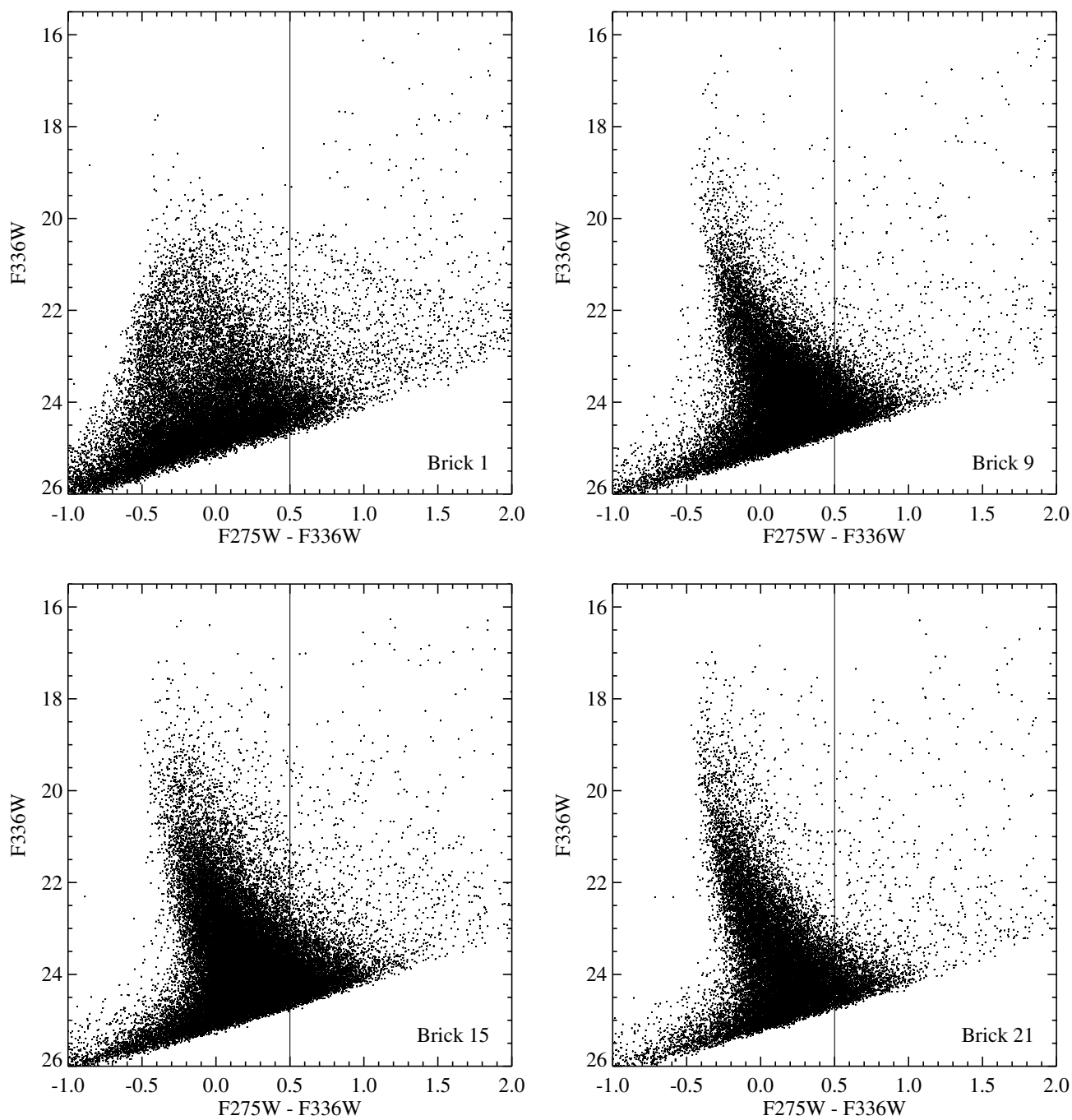

Figure 22. UV Hess diagrams for complete Bricks 1, 9, 15, and 21 (from upper left to lower right). The Hess diagram is displayed with a square root scaling to highlight both high and low density features. Outside of Brick 1, the UV CMD morphology is dominated by main-sequence stars. In the bulge-dominated Brick 1, however, a much bluer population due to hot post horizontal branch stars is also present (Rosenfield et al. 2012). The UV data reach a consistent depth across all bricks, due to the low level of crowding. The vertical line is given for reference, and matches those in Figures 20 and 21. 

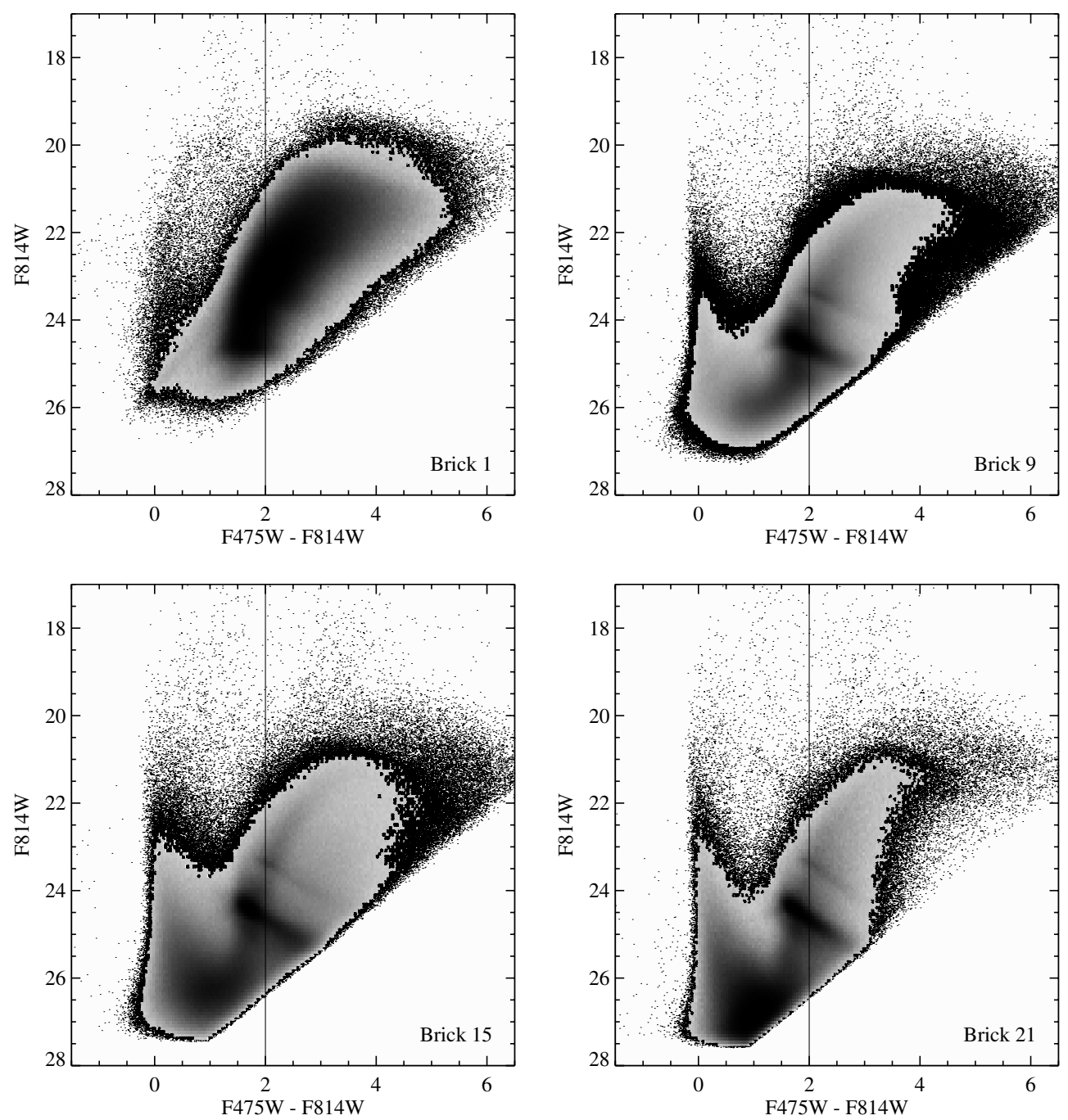

Figure 23. Optical Hess diagrams for complete Bricks 1, 9, 15, and 21 (from upper left to lower right). The Hess diagram is displayed with a square root scaling to highlight both high and low density features. The optical CMDs show a varying mixture of red RGB stars and blue main-sequence stars. The RGB also shows a clear red clump at $\mathrm{F} 814 \mathrm{~W} \sim 24.5$, and a brighter AGB bump at $\mathrm{F} 814 \mathrm{~W} \sim 23.5$. The depth in the optical depends strongly on radius because the ACS images are crowding limited at most radii. The CMD for the bulge-dominated Brick 1 also shows a small diagonal sequence extending to F814W $\sim 26$, F475W-F814W $\sim-0.5$, due to blue horizontal-branch stars (Williams et al. 2012). The vertical line is given for reference, and matches those in Figures 20 and 21.

(Figure 20, top right), assuming roughly solar metallicity for the young stellar populations. The main sequence starts to become more vertical brighter than F336W $\sim 19.5\left(\gtrsim 15 M_{\odot}\right.$ for solar-metallicity main-sequence stars) and has a color of F275W-F336W -0.45 along the blue edge of the sequence. This color is slightly redder than the color of F275W-F336W $\sim-0.6$ seen in the unreddened simulations in Figure 20, due to extinction from a Galactic foreground and dust associated with M31's star-forming regions.

The observed UV CMDs show a notable absence of stars redward of the main sequence, beyond the modest numbers expected from the Galactic foreground (Figure 21). As can be seen in Figure 20, even modestly sub-solar stellar populations can be expected to host a dramatic second sequence of blue core helium-burning stars, in addition to the main sequence. Additional simulations (not shown) suggest that there should be a clear sequence of blue core helium-burning stars present for metallicities as high as $[\mathrm{Fe} / \mathrm{H}]=-0.25$. The absence of such a sequence suggests that even the outer disk of M31 has close to solar metallicity at the present day.

In contrast to the main-sequence-dominated CMDs for Bricks 9,15 , and 21 , the bulge population in Brick 1 shows a radically different UV CMD morphology. Although a smattering of mainsequence stars are present, the most noticeable sequence is much bluer and more vertical, and dominates at colors of F275W-F336W $\sim-0.5$, fainter than F336W $\sim 20$. This sequence is made up of hot post-horizontal-branch stars that had anomalously high mass loss rates on the RGB, which then altered what would otherwise be normal AGB evolution. The properties of this population are discussed extensively in Rosenfield et al. (2012) for the PHAT data set.

The optical disk CMDs in Figure 23 show a much wider range of stellar ages than the UV (see Figure 20). Outside of the bulge (i.e., Bricks 9, 15, and 21), there is an obvious main sequence, extending nearly vertically at $\mathrm{F} 475 \mathrm{~W}-\mathrm{F} 814 \mathrm{~W} \sim 0$. The faintest detectable stars in the main sequence are found in Brick 21, and have typical masses of $\sim 1.5 M_{\odot}$, assuming solar metallicity. At redder colors, the stellar populations are dominated by a prominent RGB, punctuated by a well-populated red clump at F814W 24.5 and a noticeable AGB bump at F814W 23.5 (Gallart 1998). The RGB, red clump, and AGB bump are all smeared out diagonally by differential reddening. This effect is particularly severe in Brick 15, which covers the main starforming ring. The RGB terminates at bright magnitudes, with 
the TRGB falling at F814W 20.5 for bluer RGB stars, and $\mathrm{F} 814 \mathrm{~W} \sim 21.5$ for redder RGB stars. There is a significant population of AGB stars brightward of the TRGB, with the bulk being no more than 1.5 mag brighter than the TRGB.

There are also subtle, less populated features that can be discerned in the optical CMDs. First is a hint of a vertical sequence emerging brightward of the red clump and blueward of the RGB. This feature is most likely due to core heliumburning stars, which have characteristic ages of less than several hundred Myr (see, for example, Figure 1 of McQuinn et al. 2011). These stars typically appear as two narrow sequences in lower metallicity dwarf galaxies (see example CMDs in Dalcanton et al. 2009), but in M31 the two sequences are redder and less distinct, due both to higher metallicity and differential reddening. As a result, the "sequence" manifests more as a subtle "edge" at $\mathrm{F} 475 \mathrm{~W}-\mathrm{F} 814 \mathrm{~W} \sim 1.5$ just above the red clump, corresponding to the blue edge of the core heliumburning evolution (see the solar-metallicity panels of Figure 20). The brighter stars in the core helium-burning sequences (i.e., classical supergiants) are difficult to separate from the Galactic foreground, which overlaps their positions in the optical CMD (Figure 21; see also Drout et al. 2009), making it challenging to trace this feature to brighter magnitudes.

In addition to the core helium-burning sequences, there is a more subtle secondary overdensity of stars extending 0.5 mag faintward from the red clump, which is most evident in Brick 21 because of the lower level of crowding and better contrast in the CMD. This feature corresponds to the "secondary clump" (Girardi 1999) caused by He-burning stars which are just massive enough to have ignited $\mathrm{He}$ in non-degenerate conditions, at ages of $1 \mathrm{Gyr}$.

The optical bulge CMD in Brick 1 shows some similarities with the disk CMDs. The bulge region is dominated by a very strong RGB with a clear TRGB, and a substantial population of AGB stars populating the region brighter than the TRGB. The RGB is also very broad, which is most likely due to a significant spread in stellar metallicity, given the lack of substantial differential reddening in the bulge (e.g., Montalto et al. 2009). The crowding limit varies dramatically within different regions of Brick 1, however, such that the faint red clump is only detectable in the fields that are far from the center of the bulge.

The bluer optical populations in Brick 1 differ substantially from those seen in the disk fields in two major ways. First, there is a noticeable diagonal sequence extending blueward from the RGB at $\mathrm{F} 814 \mathrm{~W} \sim 24$, down to fainter magnitudes. In Figure 24 we overlay the post-AGB (P-AGB), post-early AGB (PE-AGB), AGB manqué, and zero-age horizontal-branch evolutionary tracks that were used to analyze the UV populations in Rosenfield et al. (2012). ${ }^{27}$ The dashed portion of the cyan line indicates a very fast interval of AGB manqué evolution. Based on these tracks, it seems most likely that the diagonal sequence is made up primarily of blue horizontal-branch stars (which are long lived), though with a possible small contribution of AGB manqué. However, an exact attribution will depend on fuller modeling that takes evolutionary lifetimes and likely reddening into account. We note that the stars in the diagonal sequence are primarily found far from the center of the bulge, but it is not yet clear if this trend is due only to the increased depth at large radii, or reflects a real population gradient; this population is being analyzed more fully in Williams et al. (2012).

\footnotetext{
27 PE-AGB and AGB manqué stars are stars that lost too much mass on the
} RGB to undergo normal AGB evolution.

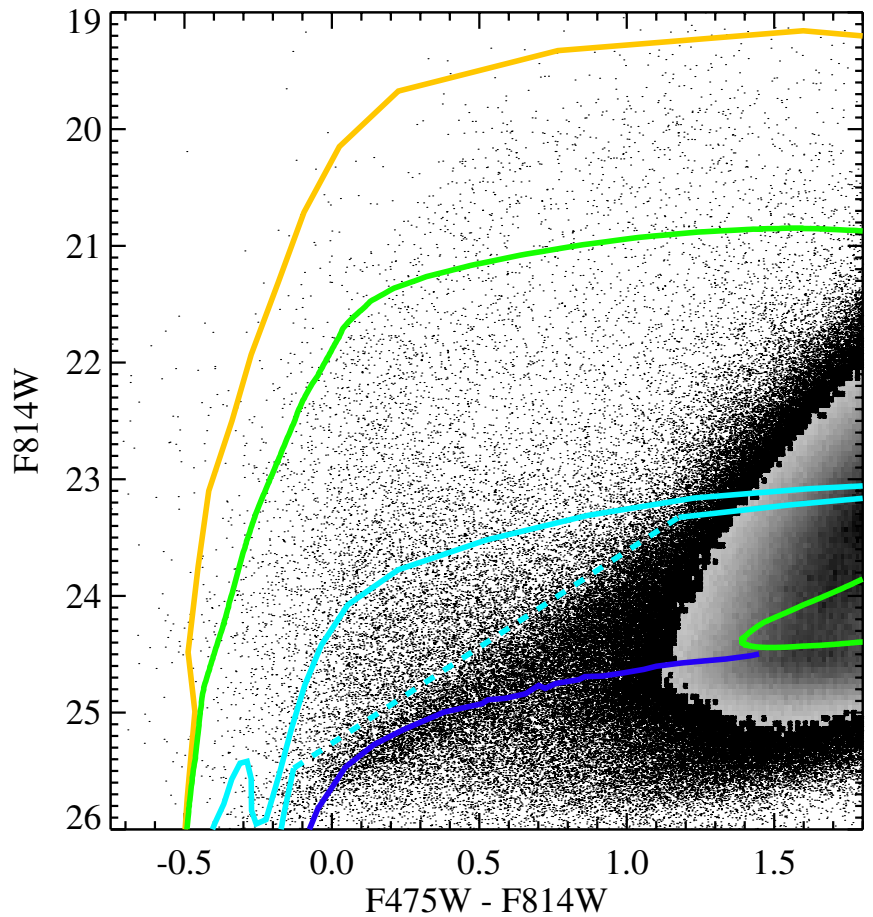

Figure 24. Optical CMD of Brick 1, focusing on the diagonal sequence of blue stars. Overlaid are evolutionary tracks from A. Bressan et al. (2012, in preparation) for post-AGB, post-early AGB, AGB manqué, and the zero-age horizontal-branch stars (plotted as orange, green, light blue, and dark blue, respectively), assuming zero foreground reddening with chemical composition $Z=0.07$ and $Y=0.389$ and an $\alpha$-enhanced composition typical of bulges (e.g., Bensby et al. 2010). We converted the tracks to the WFC3/UVIS photometric system following the Girardi et al. (2008) bolometric corrections updated with the latest WFC3/UVIS filter throughputs, and assumed $m-M=24.47$. Stars are plotted as a Hess diagram in regions of high density on the CMD; the scaling of the Hess diagram and the density level at which the Hess diagram switches to individual points has been changed from Figure 23. The dashed portion of the light blue AGB manqué track indicates a very rapid phase of evolution; while this track appears to go through the overdensity of stars, the evolution through this phase is sufficiently rapid that the actual contribution of AGB manque stars to this feature is likely to be small.

(A color version of this figure is available in the online journal.)

The second major difference between the bulge and disk's blue populations is the behavior of the blue edge of the optical CMDs. In the disk regions, the upper blue edge is defined by the nearly vertical main sequence. In Brick 1, however, there are few massive main-sequence stars. Instead, the blue edge is curved to redder colors at bright magnitudes. Comparing to the tracks in Figure 24, it appears that the blue edge in the bulge is defined by a combination of P-AGB and PE-AGB stars, with a possible contribution of main-sequence stars at fainter magnitudes.

Figure 25 shows the NIR CMDs for all four bricks. The stars in these CMDs fall primarily on the older, redder RGB and AGB sequences, with an additional small contribution from the brightest main-sequence stars. The RGB is quite narrow in all the disk fields, and a clear TRGB is present at F160W $\sim 18.3$. However, differential reddening is still apparent, even at these longer wavelengths. The effect is most easily seen in Brick 15, where large amounts of dust lead the RGB to appear doubled, due to stars seen in front of and behind the dust in the mid-plane of the galaxy. In the crowded bulge data, the RGB is broadened and does not extend more than $\sim 3$ mag below the TRGB. In the outer disk, however, the CMDs are sufficiently deep that we see both a well-populated red clump and AGB bump. These features are extended to redder, fainter colors, suggesting that there is 

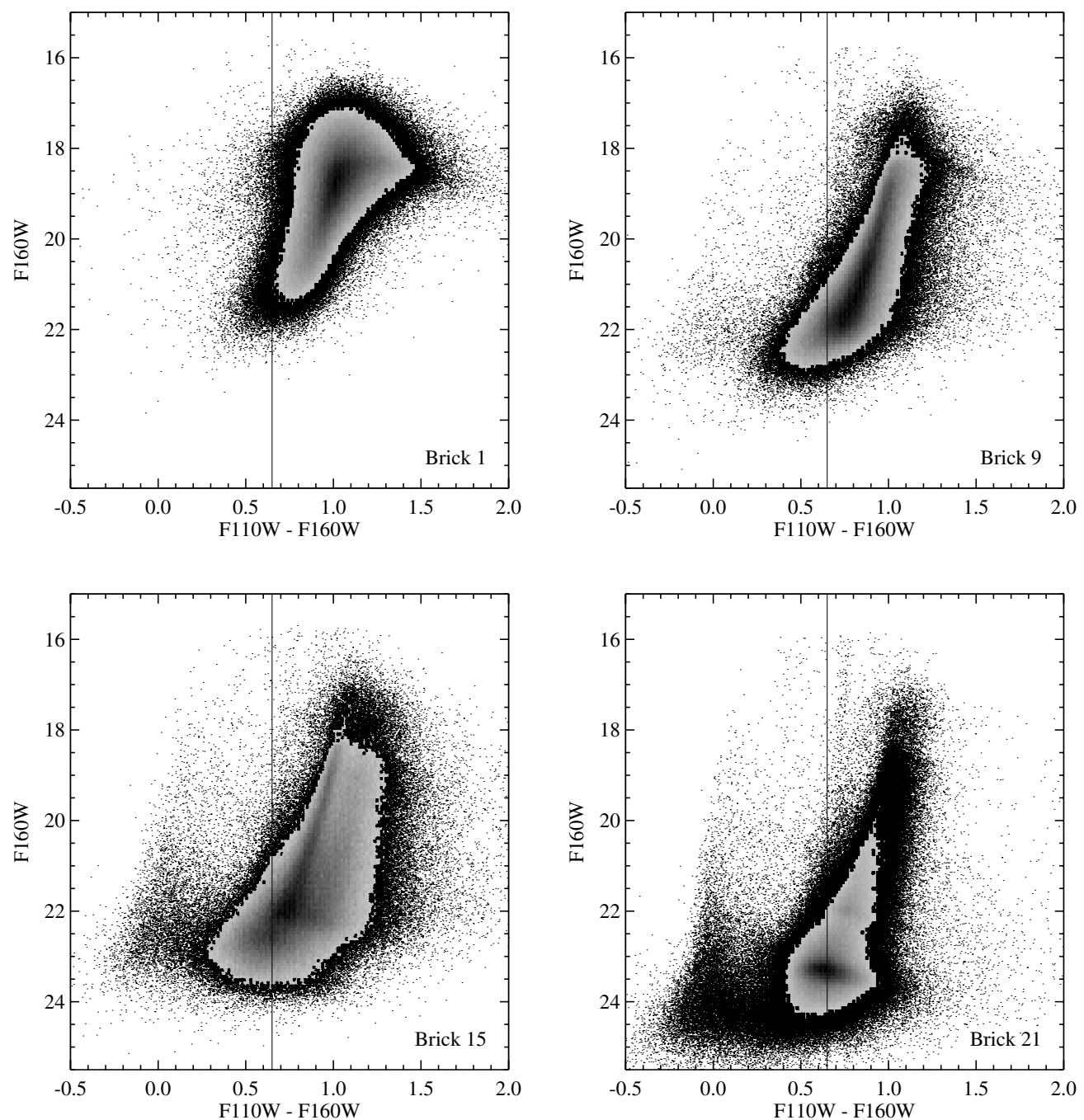

Figure 25. NIR Hess diagrams for complete Bricks 1, 9, 15, and 21 (from upper left to lower right). The Hess diagram is displayed with a square root scaling to highlight both high and low density features. The NIR CMD is dominated by RGB stars, although AGB stars and main sequence stars are also present. The depth in the NIR depends strongly on radius because the NIR images are crowding limited at all radii. In the outermost brick shown (Brick 21), the data reach below the NIR red clump (at F160W 23.5). There is also a clear AGB bump at F160W 22. Reddening and extinction blur CMD features down and to the right. The vertical line is given for reference, and matches those in Figures 20 and 21.

significant differential reddening, even at these large radii. Red core helium-burning stars are present as well, but as discussed in Dalcanton et al. (2012), they are difficult to distinguish from the main RGB and AGB sequences in this filter combination.

The PHAT bricks are sufficiently large to contain a sizable number of foreground MW stars. These appear more numerous in the NIR CMDs and appear as a narrow vertical feature at F110W-F160W $\sim 0.7$ mag. Such a feature is evident in all our NIR CMDs. Simulations with TRILEGAL (Girardi et al. 2005) reproduce well the shape and stellar density of this feature (Figure 21).

\subsection{Global Structure of the M31 Disk}

Our resolved stellar data enable us to study the spatial distribution of selected stellar populations. Here we present initial results on the spatial distribution of the abundant metal-rich $([\mathrm{Fe} / \mathrm{H}]>-0.7)$ RGB stars with ages $\gtrsim 1 \mathrm{Gyr}$. Previous studies of the structure of M31's inner disk have relied on integrated light measurements of the combined stellar population. Recent work by Courteau et al. (2011) uses I band and Spitzer 3.6 $\mu \mathrm{m}$ integrated light to fit the inner profile of M31. However, integrated light is an imperfect tracer of stellar mass, mixing stellar populations with varying mass-to-light ratios. While the NIR wavelengths used in recent work minimize the effects of dust, variations in $M / L$ ratio at NIR wavelengths from young populations are still large due to contribution from supergiant and TP-AGB stars (e.g., Melbourne et al. 2012). By using star counts rather than integrated light we can reduce the contamination of these younger populations and directly study the structure of an older, more dynamically mixed population.

We select bright RGB stars using the NIR F110W and F160W filters, as this enables detection of even highly reddened sources. Figure 26 shows an NIR CMD of stars in Bricks 8 and 9, with our metal-rich RGB selection region overlaid as a blue polygon. The blue edge of this region was selected to contain old RGB stars $(\sim 10 \mathrm{Gyr})$ up to $\sim 1$ mag below the RGB tip with metallicities $[\mathrm{Fe} / \mathrm{H}]>-0.7$. Younger RGB stars also fall within the box, with the solar metallicity $1.5 \mathrm{Gyr}$ isochrone falling along the blue edge of the selection region. The redward extension of this box follows the reddening vector and contains stars with $E(B-V) \lesssim 3$. Only the brightest RGB stars were considered so that our selection box remains well above the completeness limit over all regions except the bulge. The RGB 


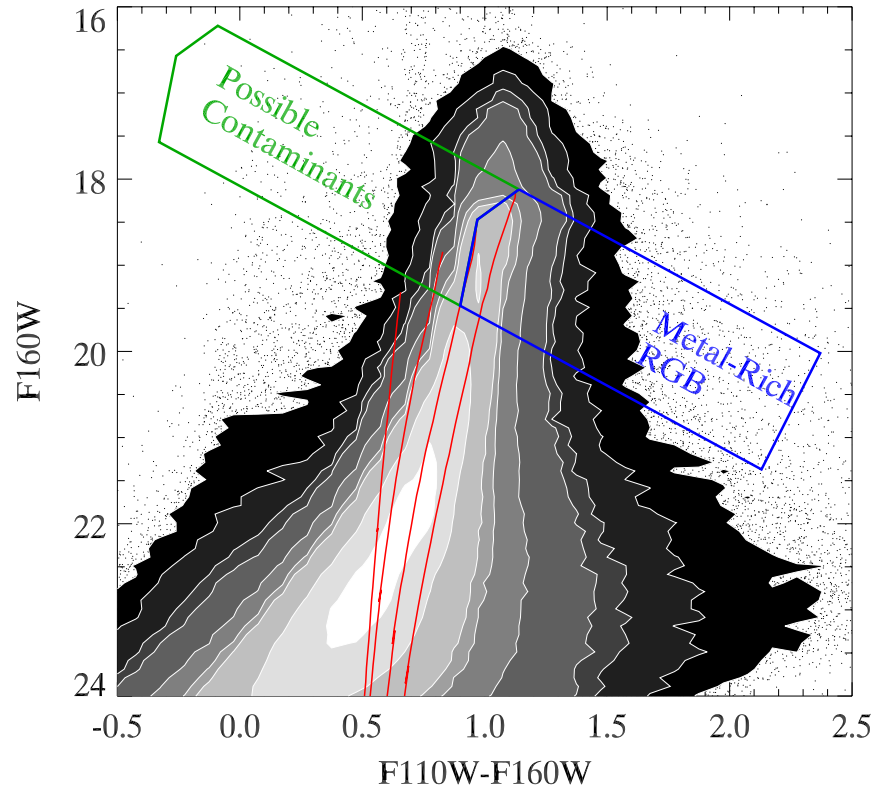

Figure 26. Example of selection of RGB stars from the NIR CMD. The blue selection region selects $\mathrm{RGB}$ stars with $[\mathrm{Fe} / \mathrm{H}]>-0.7$ and reddenings up to $E(B-V)=3$. This metal-rich RGB selection box only includes the brightest stars to ensure uniform selection of RGB stars even in the crowded inner disk (i.e., Figure 11). The green box indicates possible contaminants that could be reddened into the RGB selection area. These contaminants are metal-poor RGB stars, red supergiants, and AGB stars. This box typically contains $25 \%$ of the stars in the metal-rich selection box. Red lines indicate RGB positions for $[\mathrm{Fe} / \mathrm{H}]=(-2.3,-1.3,-0.7,0.0)$ with an age of 10 Gyr. The NIR data are drawn from the merged catalog of Bricks 8 and 9; identical selection criteria were used in all other regions. A foreground extinction correction of $E(B-V)=0.062$ was applied to the data before plotting.

(A color version of this figure is available in the online journal.)

selection box excludes younger populations such as unreddened red supergiants and TP-AGB stars.

After selecting the stars based on their position in the CMD, we calculated the densities of these stars in $30^{\prime \prime} \times 30^{\prime \prime}$ bins over all the regions with available WFC3/IR data. The RGB stellar densities are shown in the top panel of Figure 27 and range from $>1$ stars $\operatorname{arcsec}^{-2}$ in the bulge to $\sim 0.01 \mathrm{stars} \mathrm{arcsec}^{-2}$ in Brick 23. As would be expected from an old population, the RGB density distribution is quite smooth, despite the outer fields covering several regions with strong star formation or high dust extinction. The axes in Figure 27 are rotated to follow the major and minor axes assuming a position angle of $35^{\circ}$ (Paturel et al. 2003).

We fit the two-dimensional RGB density of the stellar disk to an inclined exponential disk model after applying a small correction for dust extinction discussed below. We do not attempt to fit the bulge, due to incompleteness, and exclude the central region from our fits. Initial fits to the full data yield a disk scale length of $6.1 \mathrm{kpc}$. However, this fit is clearly biased by the presence of an overdensity of RGB stars between 9 and $12.5 \mathrm{kpc}$, resulting in a model that significantly overpredicts the number of stars in Bricks 21-23 (beyond $1^{\circ}$ along the major axis). Excluding the region between 9.2 and $12.5 \mathrm{kpc}$, we obtain much better fits to the outer part of the galaxy, and a scale length of $5.3 \mathrm{kpc}$, in excellent agreement with the $5.3 \pm 0.5 \mathrm{kpc}$ found by Courteau et al. (2011). The best-fit position angle of the disk is 43.2 , with an inclination of $70^{\circ}$. This inclination is somewhat lower than the $74^{\circ}$ inclination found by Courteau et al. (2011) and the canonical value of $77^{\circ}$ assumed in earlier work (e.g., Walterbos \& Kennicutt 1988), perhaps due to our sampling an intrinsically thicker distribution of stars or our incomplete spatial coverage. The position angle agrees well with that quoted by Seigar et al. $\left(2008 ; 45^{\circ}\right)$ and is slightly rotated from the value given in Tempel et al. $(2010 ; 38.1)$. The best-fit model and residuals are shown in the middle and bottom panels of Figure 27 . The areas fitted are represented reasonably well by a single exponential disk: the reduced $\chi^{2}$ of this fit is 1.7 , assuming Poisson statistics for the RGB star counts. In contrast, inclusion of the $10 \mathrm{kpc}$ ring region results in a best-fit reduced $\chi^{2}$ of 3.7 .

Examination of the residual map in the bottom panel of Figure 27 shows a clear and significant overdensity between 9 and $12.5 \mathrm{kpc}$ along the major axis, with $\sim 40 \%$ more stars than expected in our best-fit exponential model. This overdensity is associated with the " $10 \mathrm{kpc}$ ring" seen prominently in UV emission (Thilker et al. 2005) and in the mid-IR (Barmby et al. 2006). This ring hosts ample recent star formation, and visual inspection of the PHAT data shows the expected population of young main-sequence stars in this region. However, since the stars selected for density mapping have ages $>1$ Gyr, the overdensity observed here implies that the $10 \mathrm{kpc}$ ring, originally identified through evidence for recent star formation (Habing et al. 1984; Gordon et al. 2006), is overdense in more than just recently formed stars.

A potential complication with this interpretation is that the higher extinction associated with this region could redden additional stars into our selection box. In Figure 26, the green box indicates areas of the CMD from which stars could be reddened into the selection box. This region includes primarily metal-poor RGB stars, AGB stars, and a small number of young supergiants. In the fitted region, the median number of stars in this box (referred to hereafter as $C_{\text {obs }}$ ) is $27 \%$ of the number of stars in our selection box (hereafter $R_{\mathrm{obs}}$ ). The upper envelope in $C_{\text {obs }} / R_{\text {obs }}$ is about $40 \%$. Thus, replicating the $\sim 40 \%$ overdensity seen in the $10 \mathrm{kpc}$ ring would require all of these stars to be reddened into the metal-rich RGB box. This scenario is implausible, especially given that the RGB overdensity extends over a wide range of dust extinction. We can attempt to use the observed $C_{\text {obs }} / R_{\text {obs }}$ to make a correction for the effects of dust extinction on $R_{\mathrm{obs}}$. Assuming an intrinsic $C / R$ ratio of $40 \%$, we can calculate $R_{\text {intrinsic }}$ based on the observed $C_{\mathrm{obs}} / R_{\mathrm{obs}}$ :

$$
R_{\text {intrinsic }}=R_{\mathrm{obs}} \frac{1+C_{\mathrm{obs}} / R_{\mathrm{obs}}}{1.4} .
$$

This correction does create a significantly smoother RGB density distribution, reducing the scatter in the fit residuals by $\sim 20 \%$ both in the fitted regions and in the $10 \mathrm{kpc}$ ring. This reduction in residual scatter suggests that this correction does help account for the effect of dust extinction on our number counts and therefore we have included it in our fits discussed above and shown in Figure 27. However, the correction does not lessen the significance of the $10 \mathrm{kpc}$ ring overdensity, at least in part because this region has a wide range of $C_{\mathrm{obs}} / R_{\mathrm{obs}}$. It therefore appears that the observed overdensity does in fact reflect a significant increase in the number metal-rich RGB stars at radii between 9 and $12.5 \mathrm{kpc}$ along the major axis.

In summary, we find disk scale lengths in agreement with previous estimates and find a strong overdensity ( $\sim 40 \%$ over the expected density) of RGB stars affiliated with the "10 kpc ring." These fits suggest that this structure is long lived, and not simply an enhancement of recent star formation due to a spiral arm. A much more detailed analysis of the M31 structure as a function of stellar population will be possible once the full data set is in hand. 


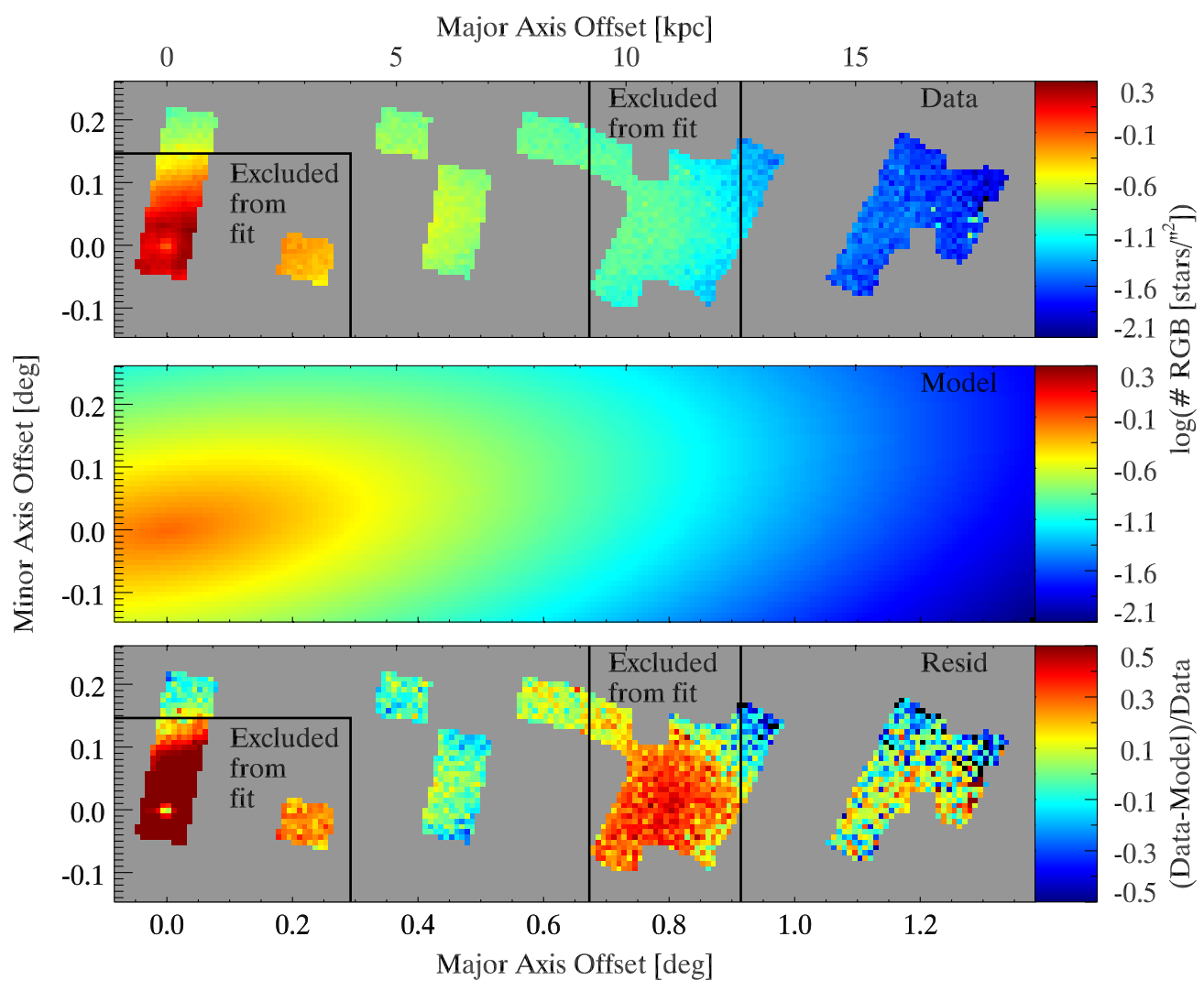

Figure 27. Top: surface density of luminous RGB stars falling in the cyan selection region from Figure 26. The overall RGB surface density distribution is quite smooth, with the exception of the inner region of the bulge, where incompleteness begins to affect even the brightest RGB stars. Middle: the best-fit single inclined exponential disk model, which was derived by fitting the observed surface density in the upper panel, after excluding the central regions of the bulge and the region from 9.2 to $12.5 \mathrm{kpc}$ along the major axis. Bottom: residual map showing the fractional deviation of the model from the data. Typical excursions are of order $10 \%$, with the exception of the $10 \mathrm{kpc}$ star-forming ring, which appears as a true enhancement in the stellar surface density of $\sim 40 \%$, and is therefore a dynamical structure, rather than simply a localized enhancement in the rate of star formation. A literature value for the position angle of $35^{\circ}$ was assumed in rotating the axes to be roughly along the major and minor axes. The fit along the major axis suggests a position angle of 43.2 .

(A color version of this figure is available in the online journal.)

\section{CONCLUSIONS}

We have presented the survey design, observations, and data products for the PHAT. We have demonstrated the superb data quality offered by this new legacy of $H S T$ observations. Given that the optical and NIR data are crowding limited over essentially all of M31's disk, the data at these wavelengths cannot be surpassed in depth until higher resolution facilities become available. Our hope is that the combination of wide wavelength coverage, photometric quality, and broad areal coverage will make this data set a rich scientific resource for decades to come.

The authors are very happy to acknowledge discussions with Jay Anderson, Tom Brown, Suzanne Hawley, and Alesandro Bressan. We are grateful to Stefano Casertano for sharing the code to produce the exposure time maps in Figure 5, Alesandro Bressan for providing the tracks plotted in Figure 24, JeanCharles Cuillandre for allowing us to use his CFHT imaging to tie our data to a global astrometric frame, to Pauline Barmby for providing us the Spitzer IRAC images, and the anonymous referee for an extremely knowledgeable and prompt report. The project has received superb support from personnel at the Space Telescope Science Institute, including Alison Vick, Ken Sembach, Neill Reid, and the ACS and WFC3 instrument teams. Zolt Levay is particularly thanked for the beautiful visualizations found in Figure 6, which far surpassed anything we were able to generate on our own. Stan Vlcek, Sarah Garner, and Pat Taylor at UW have been instrumental in helping with logistics for the project. This work was supported by the Space Telescope Science Institute through GO-12055. L.G. acknowledges support from contract ASI-INAF I/009/10/0.

\section{REFERENCES}

Anderson, J., Sarajedini, A., Bedin, L. R., et al. 2008, AJ, 135, 2055 Arp, H. 1964, ApJ, 139, 1045

Athanassoula, E., \& Beaton, R. L. 2006, MNRAS, 370, 1499

Baade, W., \& Payne-Gaposchkin, C. H. (ed.) 1963, Evolution of Stars and Galaxies (Cambridge, MA: Harvard Univ. Press)

Barmby, P., Ashby, M. L. N., Bianchi, L., et al. 2006, ApJ, 650, L45

Barmby, P., Perina, S., Bellazzini, M., et al. 2009, AJ, 138, 1667

Beaton, R. L., Majewski, S. R., Guhathakurta, P., et al. 2007, ApJ, 658, L91

Bellazzini, M., Cacciari, C., Federici, L., Fusi Pecci, F., \& Rich, M. 2003, A\&A, 405, 867

Bensby, T., Feltzing, S., Johnson, J. A., et al. 2010, A\&A, 512, A41

Bernard, E. J., Ferguson, A. M. N., Barker, M. K., et al. 2012, MNRAS, 420, 2625

Bianchi, L. 2007, in UV Astronomy: Stars from Birth to Death, ed. A. I. Gómez de Castro \& M. A. Barstow (Madrid: Editorial Completense), 65

Blair, W. P., Kirshner, R. P., \& Chevalier, R. A. 1982, ApJ, 254, 50

Bohlin, R. C. 2007, in ASP Conf. Ser. 364, The Future of Photometric, Spectrophotometric and Polarimetric Standardization, ed. C. Sterken (San Francisco, CA: ASP), 315

Bonanos, A. Z., Stanek, K. Z., Kudritzki, R. P., et al. 2006, ApJ, 652, 313

Brinks, E., \& Shane, W. W. 1984, A\&AS, 55, 179

Brown, T. M., Beaton, R., Chiba, M., et al. 2008, ApJ, 685, L121

Brown, T. M., Ferguson, H. C., Smith, E., et al. 2003, ApJ, 592, L17 
Brown, T. M., Sahu, K., Anderson, J., et al. 2010, ApJ, 725, L19

Brown, T. M., Sahu, K., Zoccali, M., et al. 2009a, AJ, 137, 3172

Brown, T. M., Smith, E., Ferguson, H. C., et al. 2006, ApJ, 652, 323

Brown, T. M., Smith, E., Ferguson, H. C., et al. 2007, ApJ, 658, L95

Brown, T. M., Smith, E., Ferguson, H. C., et al. 2009b, ApJS, 184, 152

Caldwell, N., Harding, P., Morrison, H., et al. 2009, AJ, 137, 94

Caldwell, N., Schiavon, R., Morrison, H., Rose, J. A., \& Harding, P. 2011, AJ, 141,61

Castelli, F., \& Kurucz, R. L. 2004, ArXiv:astro-ph/0405087

Chapman, S. C., Ibata, R., Lewis, G. F., et al. 2006, ApJ, 653, 255

Collins, M. L. M., Chapman, S. C., Ibata, R. A., et al. 2011, MNRAS, 413, 1548

Courteau, S., Widrow, L. M., McDonald, M., et al. 2011, ApJ, 739, 20

Cuillandre, J.-C., Lequeux, J., Allen, R. J., Mellier, Y., \& Bertin, E. 2001, ApJ, 554,190

Dalcanton, J. J., Williams, B. F., Melbourne, J. L., et al. 2012, ApJS, 198, 6

Dalcanton, J. J., Williams, B. F., Seth, A. C., et al. 2009, ApJS, 183, 67

Davidge, T. J. 2001, AJ, 122, 1386

Davidge, T. J., Olsen, K. A. G., Blum, R., Stephens, A. W., \& Rigaut, F. 2005, AJ, 129,201

Dempster, A. P., Laird, N. M., \& Rubin, D. B. 1977, J. R. Stat. Soc. B (Methodological), 39, 1

Dennefeld, M., \& Kunth, D. 1981, AJ, 86, 989

Dolphin, A. E. 2000, PASP, 112, 1383

Dolphin, A. E., Saha, A., Claver, J., et al. 2002, AJ, 123, 3154

Dorman, C., Guhathakurta, P., Fardal, M. A., et al. 2012, ApJ, in press (arXiv:1204.4455)

Driver, S. P., Allen, P. D., Liske, J., \& Graham, A. W. 2007, ApJ, 657, L85

Drout, M. R., Massey, P., Meynet, G., Tokarz, S., \& Caldwell, N. 2009, ApJ, 703,441

Durrell, P. R., Harris, W. E., \& Pritchet, C. J. 2001, AJ, 121, 2557

Fan, Z., Ma, J., de Grijs, R., \& Zhou, X. 2008, MNRAS, 385, 1973

Ferguson, A. M. N., \& Johnson, R. A. 2001, ApJ, 559, L13

Fluks, M. A., Plez, B., The, P. S., et al. 1994, A\&AS, 105, 311

Freedman, W. L., \& Madore, B. F. 1990, ApJ, 365, 186

Galarza, V. C., Walterbos, R. A. M., \& Braun, R. 1999, AJ, 118, 2775

Gallart, C. 1998, ApJ, 495, L43

Gallazzi, A., Brinchmann, J., Charlot, S., \& White, S. D. M. 2008, MNRAS, 383,1439

Gilbert, K. M., Guhathakurta, P., Kalirai, J. S., et al. 2006, ApJ, 652, 1188

Girardi, L. 1999, MNRAS, 308, 818

Girardi, L., Dalcanton, J., Williams, B., et al. 2008, PASP, 120, 583

Girardi, L., Groenewegen, M. A. T., Hatziminaoglou, E., \& da Costa, L. 2005, A\&A, 436, 895

Gordon, K. D., Bailin, J., Engelbracht, C. W., et al. 2006, ApJ, 638, L87

Guhathakurta, P., Rich, R. M., Reitzel, D. B., et al. 2006, AJ, 131, 2497

Habing, H. J., Miley, G., Young, E., et al. 1984, ApJ, 278, L59

Haiman, Z., Magnier, E. A., Battinelli, P., et al. 1994, A\&A, 290, 371

Han, S. R., Hyung, S., Park, H.-S., \& Lee, W.-B. 2001, J. Korean Astron. Soc., 34, 67

Hodge, P. W. (ed.) 1992, The Andromeda Galaxy (Astrophysics and Space Science Library, Vol. 176; Dordrecht: Kluwer)

Hodge, P. W., Krienke, O. K., Bellazzini, M., et al. 2009, AJ, 138, 770

Høg, E., Fabricius, C., Makarov, V. V., et al. 2000, A\&A, 355, L27

Hook, R., Stoehr, F., \& Krist, J. 2008, Space Telescope European Coordinating Facility Newsletter, 44, 11

Hubble, E. P. 1929, ApJ, 69, 103

Hunter, D. A., Baum, W. A., O’Neil, E. J., Jr., \& Lynds, R. 1996, ApJ, 468, 633

Jacoby, G. H., \& Ciardullo, R. 1999, ApJ, 515, 169

Jee, M. J., Blakeslee, J. P., Sirianni, M., et al. 2007, PASP, 119, 1403

Johnson, L. C., Seth, A. C., Dalcanton, J. J., et al. 2012, ApJ, in press

Kalirai, J. S., Gilbert, K. M., Guhathakurta, P., et al. 2006a, ApJ, 648, 389

Kalirai, J. S., Guhathakurta, P., Gilbert, K. M., et al. 2006b, ApJ, 641, 268

Kauffmann, G., Heckman, T. M., White, S. D. M., et al. 2003, MNRAS, 341, 54

Krienke, O. K., \& Hodge, P. W. 2007, PASP, 119, 7

Krienke, O. K., \& Hodge, P. W. 2008, PASP, 120, 1

Krist, J. 1995, in ASP Conf. Ser. 77, Astronomical Data Analysis Software and Systems IV, ed. R. A. Shaw, H. E. Payne, \& J. J. E. Hayes (San Francisco, CA: ASP), 349
Lauer, T. R. 1999, PASP, 111, 227

Lauer, T. R., Bender, R., Kormendy, J., Rosenfield, P., \& Green, R. F. 2012, ApJ, 745,121

Lindblad, B. 1956, Stockholm Obs. Ann., 19, 2

Loidl, R., Lançon, A., \& Jørgensen, U. G. 2001, A\&A, 371, 1065

Macri, L. M., Stanek, K. Z., Bersier, D., Greenhill, L. J., \& Reid, M. J. 2006, ApJ, 652, 1133

Magnier, E. A., Lewin, W. H. G., van Paradijs, J., et al. 1992, A\&AS, 96, 379

Massey, P., Armandroff, T. E., \& Conti, P. S. 1986, AJ, 92, 1303

Massey, P., Lang, C. C., Degioia-Eastwood, K., \& Garmany, C. D. 1995, ApJ, 438, 188

Massey, P., Olsen, K. A. G., Hodge, P. W., et al. 2006, AJ, 131, 2478

McConnachie, A. W., Irwin, M. J., Ferguson, A. M. N., et al. 2005, MNRAS, 356,979

McConnachie, A. W., Irwin, M. J., Ibata, R. A., et al. 2009, Nature, 461, 66

McQuinn, K. B. W., Skillman, E. D., Dalcanton, J. J., et al. 2011, ApJ, 740, 48

Melbourne, J. L., Williams, B. F., Dalcanton, J. J., et al. 2012, ApJ, 748, 47

Montalto, M., Seitz, S., Riffeser, A., et al. 2009, A\&A, 507, 283

Mould, J., Barmby, P., Gordon, K., et al. 2008, ApJ, 687, 230

Mould, J., Saha, A., \& Hughes, S. 2004, ApJS, 154, 623

Olsen, K. A. G., Blum, R. D., \& Rigaut, F. 2003, AJ, 126, 452

Olsen, K. A. G., Blum, R. D., Stephens, A. W., et al. 2006, AJ, 132, 271

Paturel, G., Petit, C., Prugniel, P., et al. 2003, A\&A, 412, 45

Perina, S., Cohen, J. G., Barmby, P., et al. 2010, A\&A, 511, A23

Ribas, I., Jordi, C., Vilardell, F., et al. 2005, ApJ, 635, L37

Richardson, J. C., Ferguson, A. M. N., Johnson, R. A., et al. 2008, AJ, 135, 1998

Richer, M. G., Stasińska, G., \& McCall, M. L. 1999, A\&AS, 135, 203

Rich, R. M., \& Mighell, K. J. 1995, ApJ, 439, 145

Rich, R. M., Mighell, K. J., Freedman, W. L., \& Neill, J. D. 1996, AJ, 111, 768

Rich, R. M., Reitzel, D. B., Guhathakurta, P., Gebhardt, K., \& Ho, L. C. 2004, AJ, 127,2139

Riess, A. G., Fliri, J., \& Valls-Gabaud, D. 2012, ApJ, 745, 156

Romaniello, M., Panagia, N., Scuderi, S., \& Kirshner, R. P. 2002, AJ, 123, 915

Rosenfield, P. A., Johnson, L. C., Girardi, L., et al. 2012, ApJ, submitted

Rubin, V. C., Krishna Kumar, C., \& Ford, W. K., Jr. 1972, ApJ, 177, 31

Saglia, R. P., Fabricius, M., Bender, R., et al. 2010, A\&A, 509, A61

Sarajedini, A., \& Jablonka, P. 2005, AJ, 130, 1627

Sarajedini, A., \& Van Duyne, J. 2001, AJ, 122, 2444

Schwarzschild, M. (ed.) 1965, Structure and Evolution of the Stars (New York: Dover)

Scowcroft, V., Bersier, D., Mould, J. R., \& Wood, P. R. 2009, MNRAS, 396 1287

Seigar, M. S., Barth, A. J., \& Bullock, J. S. 2008, MNRAS, 389, 1911

Sil'chenko, O. K., Burenkov, A. N., \& Vlasyuk, V. V. 1998, A\&A, 337, 349

Skrutskie, M. F., Cutri, R. M., Stiening, R., et al. 2006, AJ, 131, 1163

Smartt, S. J., Crowther, P. A., Dufton, P. L., et al. 2001, MNRAS, 325, 257

Stanek, K. Z., \& Garnavich, P. M. 1998, ApJ, 503, L131

Stark, A. A. 1977, ApJ, 213, 368

Stark, A. A., \& Binney, J. 1994, ApJ, 426, L31

Stephens, A. W., Frogel, J. A., DePoy, D. L., et al. 2003, AJ, 125, 2473

Tempel, E., Tamm, A., \& Tenjes, P. 2010, A\&A, 509, A91

Thilker, D. A., Hoopes, C. G., Bianchi, L., et al. 2005, ApJ, 619, L67

Trundle, C., Dufton, P. L., Lennon, D. J., Smartt, S. J., \& Urbaneja, M. A. 2002, A\&A, 395, 519

van den Bergh, S. 1991, PASP, 103, 1053

van Dokkum, P. G. 2001, PASP, 113, 1420

van Leeuwen, F., Feast, M. W., Whitelock, P. A., \& Laney, C. D. 2007, MNRAS, 379,723

Venn, K. A., McCarthy, J. K., Lennon, D. J., et al. 2000, ApJ, 541, 610

Walterbos, R. A. M., \& Kennicutt, R. C., Jr. 1988, A\&A, 198, 61

Williams, B. F. 2003, AJ, 126, 1312

Williams, B. F., Dalcanton, J. J., Gilbert, K. M., Guhathakurta, P., \& Lauer, T. R. 2012, ApJ, submitted

Worthey, G., España, A., MacArthur, L. A., \& Courteau, S. 2005, ApJ, 631, 820 Zaritsky, D. 1999, AJ, 118, 2824

Zaritsky, D., Kennicutt, R. C., Jr., \& Huchra, J. P. 1994, ApJ, 420, 87 